\title{
A flexible type system for the small Veblen ordinal
}

\author{
Florian Ranzi* Thomas Strahm**
}

October 21, 2018

\begin{abstract}
We introduce and analyze two theories for typed (accessible part) inductive definitions and establish their proof-theoretic ordinal to be the small Veblen ordinal $\vartheta \Omega^{\omega}$. We investigate on the one hand the applicative theory FIT of functions, (accessible part) inductive definitions, and types. It includes a simple type structure and is a natural generalization of S. Feferman's system $\mathrm{QL}\left(\mathrm{F}_{0}-\mathrm{IR}_{N}\right)$. On the other hand, we investigate the arithmetical theory TID of typed (accessible part) inductive definitions, a natural subsystem of $I_{1}$, and carry out a wellordering proof within TID that makes use of fundamental sequences for ordinal notations in an ordinal notation system based on the finitary Veblen functions. The essential properties for describing the ordinal notation system are worked out.

Keywords: Proof theory, inductive definitions, applicative theories, small Veblen ordinal, finitary Veblen functions, metapredicativity, higher types, subsystems of second-order arithmetic.
\end{abstract}

\section{Introduction}

In [Fef92], S. Feferman introduced a two-sorted quantificational logic and showed that it has the same strength as (Skolem's system of) primitive recursive arithmetic. The characteristics of this two-sorted quantificational logic are that it is an applicative theory augmented by type variables as the second sort and with a refined notion of comprehension terms, so-called type and function terms. In particular, this theory embodies a rule $\left(\mathrm{F}_{0}-\mathrm{R}_{N}\right)$ that is called the function-induction rule on $N$ (where $N$ is interpreted as the type for the natural numbers). It was shown to be closed under a strengthening of this rule to finitary inductively generated types $I$, called $\left(\mathrm{F}_{0}-\mathrm{IR}_{I}\right)$.

This kind of theory strongly influenced the shape of our applicative theory FIT that we are going to introduce in this article. Our motivation to examine the theory in [Fef92] was to find a natural theory for carrying out metapredicative ${ }^{1}$ wellordering proofs in the spirit of

\footnotetext{
*Institut für Informatik und angewandte Mathematik, Universität Bern, Neubrückstrasse 10, CH-3012 Bern, Switzerland. Research supported by the Swiss National Science Foundation.

** Institut für Informatik und angewandte Mathematik, Universität Bern, Neubrückstrasse 10, CH-3012 Bern, Switzerland. Email: strahm@inf .unibe.ch. Homepage: http://www.inf .unibe.ch/ ^strahm.

${ }^{1}$ The notion metapredicativity is meant in general for the approach to use proof-theoretic methods from the realm of predicative proof-theory instead of impredicative methods. In particular for wellordering proofs, we aim to avoid the use of so-called collapsing functions. We refer to [Str99] or [JKSS99]. For further reading on metapredicativity, we refer to [Jäg05] and [JS05].
} 
higher type functionals for ordinals. It seemed to provide a suitable environment for doing so. But soon, we realized that aside from this, the theory gave rise to the question of what consequence a function-induction rule for infinitary inductively generated types would have on the one side and to the idea of implementing the wellordering proofs through accessible part inductive definitions on the other side (having in mind our desire for metapredicative wellordering proofs). Hence, we tackle this question on infinitary inductively generated types only for inductively generated types that correspond to the (inductively defined) accessible part $\mathbb{I}_{\mathbb{P}, \mathbb{Q}}$ for a (binary) relation $\mathbb{Q}$ on a domain $\mathbb{P}$. In fact, our methods implicitly suggest that we get the same result for the variant where we allow for general inductively generated types.

FIT stands for "theory for function(al)s, non-iterated inductive definitions, and types (of level 1)", and it represents the first step for a generalization of the theory in [Fef92] which turns out to have the small Veblen ordinal as measure for its proof-theoretic strength, i.e., $\vartheta \Omega^{\omega}$ when using the terminology of [RW93]. Theories that have $\vartheta \Omega^{\omega}$ as proof-theoretic strength are for instance $\Pi_{2}^{1}-\mathrm{BI}_{0}$ from [RW93] or more recently $\mathrm{RCA}+\left(\Pi_{1}^{1}\left(\Pi_{3}^{0}\right)-\mathrm{CA}_{0}\right)^{-}$from [VRW17]. While these theories are analyzed by impredicative proof-theoretic methods, our treatment of FIT uses metapredicative methods for the lower bound. For the upper bound, we use an embedding into $\Pi_{3}^{1}-\mathrm{RFN}_{0}$ and get a desired upper bound result in the realm of metapredicative proof-theory due to D. Probst's modular ordinal analysis from [Pro17] that determines by metapredicative methods the proof-theoretic ordinal of various theories with strength below (and reaching to) the Bachmann-Howard ordinal $\vartheta \varepsilon_{\Omega+1}$. One of these theories is $\Pi_{3}^{1}-R_{F} N_{0}$ (which is denoted by $\mathrm{p}_{3}\left(\mathrm{ACA}_{0}\right)$ in [Pro17]) and determined to have the proof-theoretic strength of the small Veblen ordinal. Furthermore, we mention the system $\mathrm{KPi}^{0}+\left(\Pi_{3}-\mathrm{Ref}\right)$ from $[\mathrm{JS} 05]$ which is also related to $\Pi_{3}^{1}-R^{2} N_{0}$. In particular, [JS05] explains how the proof-theoretic strength of $\mathrm{KPi}^{0}+\left(\Pi_{3}-\mathrm{Ref}\right)$ can be determined to be $\vartheta \Omega^{\omega}$ by metapredicative methods.

\section{Results on the Theory FIT}

We now explain the methods used in this article for the ordinal analysis of FIT. First, we

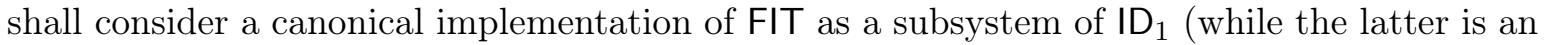
arithmetical first-order theory for non-iterated general inductive definitions, see [BFPS81]) in which metapredicative wellordering proofs can be carried out in a perspicuous way and where the interpretation back into FIT is straight-forward. This subsystem of ID $_{1}$ is called TID for "theory of typed (accessible part) inductive definitions (of level 1)" and essentially arises from $\mathrm{ID}_{1}$ by restricting to accessible part inductive definitions and adapting the closure axioms, its induction scheme on the natural numbers, and its generalized induction scheme (ID) to (the translation of) the function types of FIT, akin to the restriction of $I D_{1}$ to the theory $I D_{1}^{*} \uparrow$ from [Pro06].

For the (proof-theoretic) upper bound of FIT (and hence for TID), we shall embed it into the system $\Pi_{3}^{1}-\mathrm{RFN}_{0}$ of second-order arithmetic for $\Pi_{3}^{1} \omega$-model reflection. In order to obtain the desired upper bound $\vartheta \Omega^{\omega}$, we shall use the results from [RW93] by impredicative methods, noting that the (meta)predicative treatment from [Pro17] has not been published yet. Figure 1 depicts the abovementioned approaches accordingly. Furthermore, we give in the conclusion of this article (Section 8) some remarks on the canonical generalization of TID to a theory TID ${ }^{f}$ for general typed inductive definitions with the full range of positive arithmetical operator forms, leading to the same proof-theoretic strength of TID and $\operatorname{TID}^{\mathbf{f}}$. We refer here also to $\left[\right.$ Ran15] for an extension $\operatorname{TID}_{1}^{+}$of TID with proof-theoretic ordinal $\vartheta \Omega^{\Omega}$, i.e., the large Veblen ordinal. 


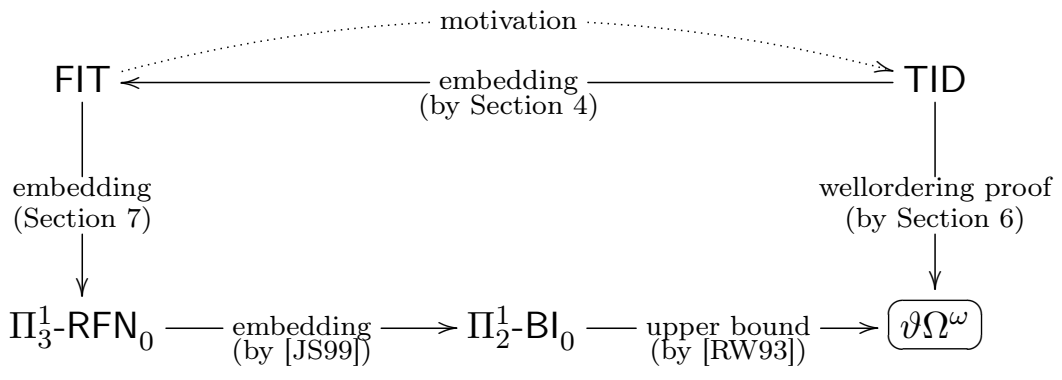

Figure 1: Strategy to determine the proof-theoretic ordinal $\vartheta \Omega^{\omega}$ of FIT

\section{General Notational Framework}

We work with three conceptually different kinds of logical frameworks: In Section 3 with the two-sorted theory FIT (with language $\mathcal{L}_{\mathrm{FIT}}$ ) that is an applicative theory enhanced by a type system; in Section 4 with the first-order theory TID (with language $\mathcal{L}_{\text {TID }}$ ) that is an extension of Peano arithmetic PA (with language $\mathcal{L}_{\mathrm{PA}}$ ) by new predicates; and in Section 7 with subsystems of second order arithmetic (with language $\mathcal{L}_{\mathrm{PA}}^{2}$ ). Hence, we work with up to two sorts of (countably many) variables and use

$$
a, b, c, d, u, v, w, x, y, z
$$

as syntactic variables for the first sort,

$$
U, V, W, X, Y, Z
$$

as syntactic variables for the second sort, and

$$
=, \neg, \rightarrow, \vee, \wedge, \exists, \forall
$$

as basic logical symbols.

Assume for now that the notion of $\mathcal{L}$ terms and $\mathcal{L}$ formulas has been already introduced for $\mathcal{L}$ being one of the languages $\mathcal{L}_{\mathrm{FIT}}, \mathcal{L}_{\mathrm{TID}}, \mathcal{L}_{\mathrm{PA}}$, or $\mathcal{L}_{\mathrm{PA}}^{2}$. IF $\mathcal{L}$ is clear from the context, we may drop the reference to $\mathcal{L}$ by just using the notions term and formula. We use $s, t, r$ as syntactic variables for $\mathcal{L}$ terms and $A, B, C, D, E, F$ as syntactic variables to denote $\mathcal{L}$ formulas. Literals are atomic $\mathcal{L}$ formulas or their negated version. Introducing an $\mathcal{L}$ formula as $A(a)$ means $A$ denotes this formula and that the variable $a$ may occur freely in $A$. $\mathrm{FV}(A)$ denotes the set of free variables of the first sort of $A$. We shall use the following notational conventions that $a, b, c, d, u, v, w$ denote free variables of the first sort within an $\mathcal{L}$ formula and that $k, l, m, n, p, q$ denote variables in our meta-theory, i.e., as ranging there over the natural numbers. Parentheses may be used to make expressions unambiguous or more readable. We prefer infix notation rather than prefix notation when dealing with binary function and relation symbols. For $\rightarrow$, we follow the usual convention of right-associativity, e.g., $A \rightarrow B \rightarrow C$ denotes $A \rightarrow(B \rightarrow C)$. We write $A \leftrightarrow B$ to denote $(A \rightarrow B) \wedge(B \rightarrow A)$. Moreover, $\wedge$ binds stronger than $\rightarrow$. 


\section{Vector Notations}

If $*$ denotes one of the syntactic variables that will be introduced in this article, then we allow the usual annotations such as $*^{\prime}, \tilde{*}$, or subscripts $*_{i}$ (for $i \in \mathbb{N}$, i.e., for natural numbers $i$ ).

For subscripts, we also use the vector notation $\vec{*}$ to denote lists of the form $*_{1}, \ldots, *_{n}$ for some $n \in \mathbb{N}$. Moreover, when working with ordinal notations based on the finitary Veblen functions, the following notation will come in handy. We write

$$
\bar{*}^{(n)}
$$

for $n \in \mathbb{N}$ to denote the list $*_{1}, \ldots, *_{n}$, and we may write $\bar{*}^{(k)}$ for any $k \in \mathbb{N}$ in order to denote $*_{1}, \ldots, *_{\min (k, n)}$. In rare cases we write for specific constants $c$ (e.g., for 0$)$ the expression $\bar{c}^{(n)}$ to denote the list $c, \ldots, c$ of length $n$, and hence we read $\vec{c}$ analogously. If $n=0$, then $\bar{*}^{(n)}$ and $\vec{*}$ denote the empty list.

Applications of all these notations will be obvious, following common conventions - for instance $\forall \bar{x}^{(3)} A$ shall abbreviate $\forall x_{1} \forall x_{2} \forall x_{3} A$ as usual, and $\forall \vec{x} A$ is just $A$ if $\vec{x}$ is the empty list. Also when writing $f \bar{t}^{(n)}$ for a list of terms $\bar{t}^{(n)}$ and an $n$-ary function symbol $f$, it is usually meant to abbreviate $f t_{1} \ldots t_{n}$ rather than $f t_{1}, \ldots, t_{n}$.

\section{Class Terms and Substitution}

$\mathcal{L}$ class terms are objects of the form

$$
\Lambda a . A
$$

for any $\mathcal{L}$ formula $A$ and we use $\mathcal{A}, \mathcal{B}, \mathcal{C}, \mathcal{D}$ as syntactic variables for $\mathcal{L}$ class terms. Sometimes, class terms are also called comprehension terms, and we do not use the more common notations $\{a: A\}$ or $\lambda a . A$ because these notions are already reserved in our setting of the applicative theory FIT.

Substitution of a variable $a$ in an $\mathcal{L}$ formula $A$ by an $\mathcal{L}$ term $t$ is denoted by $A(t / a)$ and $A_{a}(t)$, or just by $A(t)$ in case $A$ has been introduced in the form $A(a)$, and as usual we assume (if necessary) an appropriate renaming of bound variables in $A$ to avoid a clash of bound variables. Then for $\mathcal{A}$ being $\Lambda a$.A, we set

$$
\mathcal{A}(t):=t \in \mathcal{A}:=A(t / a)
$$

for any $\mathcal{L}$ term $t$ and write ambiguously $\mathcal{A} \in \mathcal{L}$ to stress that $\mathcal{A}$ is an $\mathcal{L}$ class term. Moreover, we also extend this to lists of variables $\vec{a}=a_{1}, \ldots, a_{n}$ and have objects of the form $\Lambda a_{1} \ldots \Lambda a_{n}$.A or $\Lambda \vec{a} . A$ for short with $(\Lambda \vec{a} . A)(\vec{t}):=A(\vec{t} / \vec{a})$ for terms $\vec{t}=t_{1}, \ldots, t_{n}$ and where $A(\vec{t} / \vec{a})$ is obtained by simultaneously replacing in $A$ all free occurrences of the variables $\vec{a}$ by $\vec{t}$, while renaming bound variables may be necessary to avoid a clash of variables. Analogously for $k<n$, we use $\left(\Lambda \bar{a}^{(n)} \cdot A\right)\left(\bar{t}^{(k)}\right)$ to denote $\Lambda a_{k+1} \ldots \Lambda a_{n} \cdot\left(A\left(\bar{t}^{(k)} / \bar{a}^{(k)}\right)\right)$ while tacitly assuming a reasonable variable-renaming to avoid clashes of variables.

In case that $\mathcal{L}$ also embodies variables $X, Y, Z$ of the second sort, we mean by substitution of a variable $X$ in an $\mathcal{L}$ formula $A$ by an $\mathcal{L}$ class term $\mathcal{B}$ the expression $A(\mathcal{B} / X)$ which is obtained from $A$ by substituting any atomic formula $X t$ with $\mathcal{B}(t)$ while a renaming of bound variables may be necessary as usual. If $A$ has been introduced in the form $A(X)$, we may also just write $A(\mathcal{B})$ for $A(\mathcal{B} / X)$. 
In case $R$ is a unary relation symbol in $\mathcal{L}$ or a second sort variable, we also define $A(R / X):=$ $A(\mathcal{B} / X)$ for $\mathcal{B}:=\Lambda a . R a$. Furthermore, if $\mathcal{A}$ is an $\mathcal{L}$ class term $\Lambda a$.A, then we set $\mathcal{A}(\mathcal{B} / X):=$ $\Lambda a \cdot A(\mathcal{B} / X)$. Accordingly, we let substitution for number variables be defined by $\mathcal{A}_{z}(t):=$ $\mathcal{A}(t / z):=\Lambda a \cdot A(t / z)$ assuming a renaming of the variable $a$ in case of clashes of variables.

\section{The Theory FIT for Functions, Inductive Definitions, and Types}

We introduce a basic language that is needed for the applicative part of FIT before defining the full language $\mathcal{L}_{\mathrm{FIT}}$ in Subsection 3.1.

Definition 3.1. The following basic language of FIT is based on a first sort of variables called individual variables and a second sort variables called type variables.

(a) Constants of the first sort:

$\mathrm{k}, \mathrm{s}, \mathrm{p}, \mathrm{p}_{0}, \mathrm{p}_{1}, 0, \mathrm{~s}_{\mathrm{N}}, \mathrm{p}_{\mathrm{N}}, \mathrm{d}_{\mathrm{N}}$, denoting the usual applicative constants.

(b) Constants of the second sort:

$\mathrm{N}, \overline{\mathrm{N}}, \mathrm{U}$, for the natural numbers, its complement, and a symbol without further interpretation but needed for proof-theoretic investigations.

(c) Relation symbols of the first sort:

$=, \downarrow$, denoting equality and definedness for individual terms ${ }^{2}$.

(d) Further symbols:

$\cdot, \in$, denoting a binary function symbol for first sort term application and a binary relation symbol between individual terms and types ${ }^{3}$.

Definition 3.2. Individual terms $s, t, r$ are defined inductively from individual variables and constants by use of the binary function symbol $\cdot$ as usual.

Definition 3.3. The following notions and abbreviations will serve as basic applicative tools.

(a) $t^{\prime}:=\mathrm{s}_{\mathrm{N}} t$ and $1:=0^{\prime}$.

(b) $s t_{1} \ldots t_{n}$ or also $s\left(t_{1}, \ldots, t_{n}\right)$ denotes term application on $n$ inputs and is defined recursively on $n \geq 0$ as $s$ for $n=0$ and $\left(s \cdot t_{1}\right) t_{2} \ldots t_{n}$ otherwise.

(c) $\left\langle s_{0}, \ldots, s_{n-1}\right\rangle^{\mathrm{FIT}}$ denotes general $n$-tupling and is defined recursively on $n \geq 0$ as 0 if $n=0$ and $\mathrm{p} s_{0}\left\langle s_{1}, \ldots, s_{n-1}\right\rangle^{\mathrm{FIT}}$ otherwise. Write also $\left\langle s_{0}, \ldots, s_{n-1}\right\rangle$ if the meaning is clear from the context (e.g., in the proof of Lemma 4.23).

(d) $(s)_{n}^{\mathrm{FIT}}$ denotes $n$-th projection and is defined recursively on $n \geq 0$ as $\mathrm{p}_{0} s$ if $n=0$ and $\left(\mathrm{p}_{1} s\right)_{n-1}^{\mathrm{FIT}}$ otherwise. Write also $(s)_{n}$ if the meaning is clear from the context.

(e) $\lambda x . t$ denotes lambda abstraction of a variable $x$ on a term $t$ and is defined in the usual way by making use of the combinators s and $\mathrm{k}$, while $\lambda x$.t does not contain the variable $x$. In general, $\lambda \vec{x} . t$ for a list of variables $\vec{x}=x_{1}, \ldots, x_{n}$ denotes recursively $\lambda x_{1} .\left(\lambda x_{2} \ldots x_{n} . t\right)$ if $n>0$ and $t$ otherwise. See [Ran15, Definition 5.3.(e)] for details.

\footnotetext{
${ }^{2}$ Individual terms will be defined in Definition 3.2

${ }^{3}$ Types will be defined in Definition 3.4.
} 


\subsection{Syntax of FIT}

Definition 3.4. The language $\mathcal{L}_{\mathrm{FIT}}$ is defined simultaneously and inductively with the notions for formulas (For), positive formulas $\left(\right.$ For $\left.^{+}\right)$, types (Ty), restricted types (Ty $\uparrow$ ), and terms of the second sort:

(a) $\mathcal{L}_{\mathrm{FIT}}$ extends the basic language from Definition 3.1 by the following new (syntactically different) kinds of terms of the second sort while demanding $A \in \mathrm{For}^{+}$and $\mathbb{P}, \mathbb{Q} \in \mathrm{Ty} \uparrow$ :

$$
\{x: A\} \quad \text { and } \quad \mathbb{I}_{\mathbb{P}, \mathbb{Q}}
$$

(b) For denotes the collection of formulas $A, B, C, D$, which consists of the expressions

$$
\begin{array}{rl}
t \in \mathbb{P} \quad t \in U & t \downarrow \quad s=t \\
\neg A \quad A \rightarrow B \quad A \vee B \quad & A \wedge B \quad \exists x A \quad \forall x A \quad \exists X A \quad \forall X A
\end{array}
$$

and we demand here $\mathbb{P} \in \mathrm{Ty}$. We sometimes write $A \in \mathcal{L}_{\mathrm{FIT}}$ ambiguously for $A \in$ For.

(c) $\mathrm{For}^{+}$denotes the collection of positive (elementary) formulas, i.e., $A \in$ For such that

- quantifications of type variables do not occur and

- expressions of the form $t \in \mathbb{P}$ for types $\mathbb{P} \in$ Ty occur at most positively. ${ }^{4}$

(d) Ty denotes the collection of types $\mathbb{P}, \mathbb{Q}, \mathbb{R}$ (also called positive types), i.e., expressions

$$
\begin{aligned}
& X, Y, Z, \ldots \text { (i.e., type variables) } \\
& \begin{array}{lllll}
\mathrm{N} & \overline{\mathrm{N}} & \{x: A\} & \mathbb{I}_{\mathbb{P}, \mathbb{Q}}
\end{array}
\end{aligned}
$$

while demanding $A \in \mathrm{For}^{+}$and $\mathbb{P}, \mathbb{Q} \in \mathrm{Ty} \uparrow$. Note that $\mathrm{U}$ itself is not treated as a type.

(e) Ty $\uparrow$ denotes the collection of restricted types, i.e., types such that

- no type variables and

- no expressions of the form $\mathbb{I}_{\mathbb{P}, \mathbb{Q}}$ occur

Definition 3.5. Let $\rightarrow$ be a new distinguished symbol in our meta-language. The collection FT of function types $\mathbb{F}, \mathbb{G}, \mathbb{H}$ denotes objects in the meta-language that are defined inductively to consist of expressions of the form

$$
\left(\mathbb{P}_{1} \rightarrow\left(\ldots\left(\mathbb{P}_{n-1} \rightarrow \mathbb{P}_{n}\right) \ldots\right)\right)
$$

for any $\mathbb{P}_{1}, \ldots, \mathbb{P}_{n} \in \mathrm{Ty}$. We let $\rightarrow$ be right-associative and we write just $\mathbb{P}_{1} \rightarrow \ldots \mathbb{P}_{n-1} \rightarrow \mathbb{P}_{n}$.

Remark 3.6. Note that function types are defined as objects in the meta-language. Within $\mathcal{L}_{\mathrm{FIT}}$ formulas, these new expressions will only occur in combination with terms in the form $t \in \mathbb{P} \rightarrow \mathbb{F}$, i.e., as abbreviations for proper $\mathcal{L}_{\mathrm{FIT}}$ formulas as in Notation 3.8. For remarks on how to make function types first-class members of $\mathcal{L}_{\mathrm{FIT}}$, see [Ran15, Remark 5.10.(b)] and [Fef92]. Further, we do not need $\mathrm{U}$ to be a type because $t \in \mathrm{U}$ can be obtained from $\{x: x \in \mathrm{U}\}$ and $\left(\mathrm{CA}^{+}\right)$in FIT (see Definition 3.10).

\footnotetext{
${ }^{4}$ Positive is meant in the usual way: $t \in \mathbb{P}$ is called positive in $A \in$ For if it does not occur in negated form $\neg(t \in \mathbb{P})$ in $A^{\prime}$, while $A^{\prime}$ shall be the translation of $A$ where first each subformula of the form $B_{1} \rightarrow B_{2}$ is transformed to $\neg B_{1} \vee B_{2}$ and where we then move the negation symbol $\neg$ next to atomic formulas, while making use of De Morgan's laws and the law of double negation.
} 
Definition 3.7. The notion of $\mathrm{FV}(A)$ is extended to the notion of atomic formulas $t \in \mathbb{P}$ for $\mathbb{P} \in$ Ty recursively in the canonical way as the union of the expressions involved. In particular, $I_{\mathbb{P}^{\prime}, \mathbb{Q}^{\prime}}$ is $\mathrm{FV}\left(\mathbb{P}^{\prime}\right) \cup \mathrm{FV}\left(\mathbb{Q}^{\prime}\right)$. With this extension explained, the substitution of individual and type variables is defined as in Section 2.

Notation 3.8. We have the following abbreviations for some formulas and types:

- $s \simeq t$ is $(s \downarrow \vee t \downarrow) \rightarrow s=t$.

- $s \neq t$ is $s \downarrow \wedge t \downarrow \wedge \neg(s=t)$.

- $t \in \mathbb{P} \rightarrow \mathbb{F}$ is recursively $\forall x(x \in \mathbb{P} \rightarrow t x \in \mathbb{F})$.

- $\mathrm{N}^{n+1} \rightarrow \mathbb{F}$ is recursively $\mathrm{N} \rightarrow\left(\mathrm{N}^{n} \rightarrow \mathbb{F}\right)$ where $\mathrm{N}^{0} \rightarrow \mathbb{F}$ is $\mathbb{F}$.

- $t \notin \mathbb{F}$ is $\neg(t \in \mathbb{F})$.

- $(\exists x \in \mathbb{F}) B$ is $\exists x(x \in \mathbb{F} \wedge B)$.

- $(\forall x \in \mathbb{F}) B$ is $\forall x(x \in \mathbb{F} \rightarrow B)$.

- $\mathrm{Cl}_{\mathbb{P}, \mathbb{Q}}(\mathcal{A})$ is $\forall x((x \in \mathbb{P} \wedge(\forall y \in \mathbb{P})(\langle y, x\rangle \in \mathbb{Q} \rightarrow \mathcal{A}(y))) \rightarrow \mathcal{A}(x))$.

We assume as usual for such notational abbreviations that $x, y$ are supposed to not occur in $\mathcal{A}, \mathbb{P}$, and $\mathbb{Q}$. This shall hold analogously for similar such abbreviations for formulas.

- $A(\mathbb{F} / X)$ for the formula obtained by substituting any occurrence of $t \in X$ in $A$ by $t \in \mathbb{F}$.

\subsection{The Theory FIT}

Definition 3.9. The logic of FIT is a two-sorted logic whose first-order part (i.e., for individual variables) is based on the classical logic of partial terms LPT due to Beeson [Bee85]:

- Propositional axioms and rules. The usual propositional axioms and rules, based on some sound Hilbert calculus for classical propositional logic.

- Quantificational logic for the first sort. For $A$ being an $\mathcal{L}_{\mathrm{FIT}}$ formula and $t$ an individual term, we have

$$
(\forall x A \wedge t \downarrow) \rightarrow A(t / x) \quad(A(t / x) \wedge t \downarrow) \rightarrow \exists x A
$$

and for $A, B$ being $\mathcal{L}_{\mathrm{FIT}}$ formulas and $x \notin \mathrm{FV}(A)$, we have the following figures:

$$
\frac{A \rightarrow B}{A \rightarrow \forall x B} \quad \frac{B \rightarrow A}{\exists x B \rightarrow A}
$$

- Quantificational logic for the second sort. For $A, B$ being $\mathcal{L}_{\mathrm{FIT}}$ formulas and $\mathbb{P}$ a type, we have

$$
\forall X A \rightarrow A(\mathbb{P} / X) \quad A(\mathbb{P} / X) \rightarrow \exists X A
$$

and for $A, B$ being $\mathcal{L}_{\mathrm{FIT}}$ formulas and $X$ not occurring free in $A$, we have the following figures:

$$
\frac{A \rightarrow B}{A \rightarrow \forall X B} \quad \frac{B \rightarrow A}{\exists X B \rightarrow A}
$$


- Equality axioms.

$$
x=x \quad\left(x_{1}=y_{1} \wedge \ldots \wedge x_{n}=y_{n} \wedge A\right) \rightarrow\left(\ldots\left(A\left(y_{1} / x_{1}\right)\right) \ldots\left(y_{n} / x_{n}\right)\right)
$$

- Definedness axioms. For constants $c$ of the first sort of $\mathcal{L}_{\mathrm{FIT}}$, types $\mathbb{P}$, and individual terms $t$, we have

$$
c \downarrow \wedge x \downarrow \quad(s t) \downarrow \rightarrow(s \downarrow \wedge t \downarrow) \quad s=t \rightarrow(s \downarrow \wedge t \downarrow) \quad t \in \mathbb{P} \rightarrow t \downarrow \quad t \in \mathrm{U} \rightarrow t \downarrow
$$

Writing $\vdash A$ for any $\mathcal{L}_{\mathrm{FIT}}$ formula $A$ denotes the derivability of $A$ in the logic of FIT.

Definition 3.10. FIT is the two-sorted applicative theory based on the logic of partial terms LPT (and on [Fef92]). Its non-logical axioms are as follows:

I. Applicative axioms.

I.1. Partial combinatory algebra.

$\mathrm{k} x y=x \quad \mathrm{~s} x y \downarrow \wedge \mathrm{s} x y z \simeq(x z)(y z)$

\section{I.2. Pairing and projection.}

$\mathrm{p}_{0}(\mathrm{p} x y)=x \wedge \mathrm{p}_{1}(\mathrm{p} x y)=y$

I.3. Definition by numerical cases.

$x \in \mathrm{N} \wedge y \in \mathrm{N} \wedge x=y \rightarrow \mathrm{d}_{\mathrm{N}} z_{1} z_{2} x y=z_{1} \quad x \in \mathrm{N} \wedge y \in \mathrm{N} \wedge x \neq y \rightarrow \mathrm{d}_{\mathrm{N}} z_{1} z_{2} x y=z_{2}$

I.4. Axioms about $\mathrm{N}$ and $\bar{N}$.

$$
\begin{array}{ll}
0 \in \mathrm{N} \wedge\left(x \in \mathrm{N} \rightarrow x^{\prime} \in \mathrm{N}\right) & x \in \mathrm{N} \rightarrow\left(x^{\prime} \neq 0 \wedge \mathrm{p}_{\mathrm{N}}\left(x^{\prime}\right)=x\right) \\
(x \in \mathrm{N} \wedge x \neq 0) \rightarrow\left(\mathrm{p}_{\mathrm{N}} x \in \mathrm{N} \wedge\left(\mathrm{p}_{\mathrm{N}} x\right)^{\prime}=x\right) & x \in \overline{\mathrm{N}} \leftrightarrow x \notin \mathrm{N}
\end{array}
$$

II. Induction on $\mathrm{N}$ for $\mathbb{F} \in \mathrm{FT}$.

$($ FT-Ind $) \quad t 0 \in \mathbb{F} \wedge(\forall x \in \mathrm{N})\left(t x \in \mathbb{F} \rightarrow t x^{\prime} \in \mathbb{F}\right) \rightarrow t \in(\mathrm{N} \rightarrow \mathbb{F})$

III. Positive comprehension for $A \in$ For $^{+}$.

$\left(\mathrm{CA}^{+}\right) \quad y \in\{x: A\} \leftrightarrow A(y / x)$

IV. Axioms about $I_{\mathbb{P}, \mathbb{Q}}$ for $\mathbb{F} \in \mathrm{FT}$ and $\mathbb{P}, \mathbb{Q} \in \mathrm{Ty} \uparrow$.

$(\mathrm{FT}-\mathrm{Cl}) \quad \mathrm{Cl}_{\mathbb{P}, \mathbb{Q}}\left(\Lambda z . z \in \mathrm{I}_{\mathbb{P}, \mathbb{Q}}\right)$

$(\mathrm{FT}-\mathrm{ID}) \quad \mathrm{Cl}_{\mathbb{P}, \mathbb{Q}}(\Lambda z . t z \in \mathbb{F}) \rightarrow t \in\left(\mathrm{I}_{\mathbb{P}, \mathbb{Q}} \rightarrow \mathbb{F}\right)$

Writing FIT $\vdash A$ for any $\mathcal{L}_{\mathrm{FIT}}$ formula $A$ denotes the derivability of $A$ from these axioms in the logic of FIT given in Definition 3.9.

\section{Lemma 3.11.}

(a) Lambda abstraction: For all $\mathcal{L}_{\mathrm{FIT}}$ terms $t, s$ and $\vec{s}=s_{1}, \ldots, s_{n}$, and all individual variables $y$ and $\vec{x}=x_{1}, \ldots, x_{n}$ with $y \notin\left\{x_{1}, \ldots, x_{n}\right\}$, we have the following:

1. $\mathrm{FIT} \vdash(\lambda \vec{x} . t) \downarrow \wedge(\lambda \vec{x} . t) \vec{x} \simeq t$. 
2. $\mathrm{FIT} \vdash\left(s_{1} \downarrow \wedge \ldots \wedge s_{n} \downarrow\right) \rightarrow(\lambda \vec{x} . t) \vec{s} \simeq t(\vec{s} / \vec{x})$.

3. FIT $\vdash(\lambda \vec{x} . t)(s / y) x \simeq(\lambda \vec{x} . t(s / y)) x$.

(b) Fixed-point: There exists a closed term fix such that FIT $\vdash$ fix $\downarrow \wedge$ fixy $x \simeq y($ fix $y) x$ holds for all number variables $x, y$.

(c) Pairs and tupling: For all $\mathcal{L}_{\mathrm{FIT}}$ variables $x_{0}, \ldots, x_{n}$ and each $0 \leq i \leq n$, we have FIT $\vdash\left(s_{0} \downarrow \wedge \ldots \wedge s_{n} \downarrow\right) \rightarrow\left(\left\langle s_{0}, \ldots, s_{n}\right\rangle\right)_{i}=s_{i}$.

Proof. The applicative part of FIT corresponds to the standard axioms and constants that appear in applicative theories. For details on (a) and (b), see for instance [Fef79]. In particular for (c), we remark that this follows easily from the axioms of FIT, in particular by making use of I.2. from Definition 3.10 and the definedness axioms.

\subsection{Informal Interpretation of FIT}

Since FIT directly evolved from Feferman's theory $\mathrm{QL}\left(\mathrm{F}_{0}-\mathrm{IR}_{N}\right)$, we refer for a thorough motivation and informal interpretation of FIT to [Fef92, Sections 2 and 5]. Moreover, the special constant $\mathrm{U}$ can be interpreted as a subset of the natural numbers, having no further interpretation. It is needed for proof-theoretic investigations.

Regarding the definition of axiom (FT-ID), we can motivate this as an induction principle with respect to the totality notion of a (type 1) function, while noting hereby the restriction $\mathbb{F} \in \mathrm{FT}$ in the definition of (FT-ID). The restriction $\mathbb{P}, \mathbb{Q} \in \mathrm{Ty} \uparrow$ can be seen as accounting for non-iterated inductive definitions.

\section{The Theory TID for Typed Inductive Definitions}

FIT is a natural theory for specifying the behaviour of an applicative term $t$ by use of types, say by a function type $\mathbb{P}_{1} \rightarrow \ldots \rightarrow \mathbb{P}_{n+1}$ that consists of types. For checking this behaviour, we have the axiom schemes (FT-Ind) and (FT-ID) at hand. The latter allows the discussion of the behaviour of an operation $t$ that acts on the inductively defined accessible part of a given binary relation (e.g., if $\mathbb{P}_{1}$ is $\mathbb{I}_{\mathbb{P}, \mathbb{Q}}$ in the example above). This gives an idea for the following definition of the theory TID for typed inductive definitions as a subtheory of ID $_{1}$. Before turning to the definition of TID, we introduce basic notions that we are going to use in combination with arithmetical theories.

Definition 4.1. $\mathcal{L}_{\mathrm{PA}}$ is the first-order language of Peano arithmetic with just one sort of variables $x$, referred to as (number) variables, a unary relation symbol $\mathrm{U}$ (without further interpretation and that is needed for proof-theoretic investigations), a symbol = for equality, function symbols for all primitive recursive functions (and we denote by $\mathrm{PR}^{n}$ the collection of those function symbols that have arity $n \in \mathbb{N}$ ). Moreover, $\mathcal{L}_{\mathrm{PA}}$ contains for each function symbol $f \in \mathrm{PR}^{n}$ with $n \neq 0$ also relation symbols $R_{f}$ of arity $n$. Let $\mathrm{PR}:=\bigcup_{n \in \mathbb{N}} \mathrm{PR}^{n}$ and see [Ran15, Definition 1.1.] for more details.

The relation symbols $R_{f}$ in $\mathcal{L}_{\mathrm{PA}}$ are needed for technical reasons to ease the embedding from TID into FIT in Section 4.2 (cf., Remark 4.20).

Definition 4.2. The language $\mathcal{L}_{\mathrm{PA}}^{2}$ denotes the language of second-order arithmetic, i.e., the extension of $\mathcal{L}_{\mathrm{PA}}$ by a second sort of variables $X$, referred to as set variables or just sets. 
Definition 4.3. The usual operations on the natural numbers are denoted as follows: $\mathbf{S}$ is the successor function, $0_{\mathbb{N}}$ is the zero constant, $+_{\mathbb{N}}$ is the binary function symbol for addition, $<_{\mathbb{N}}$ is the binary less-than relation. Then, $1_{\mathbb{N}}, 2_{\mathbb{N}}, \ldots$ abbreviate $\mathbf{S} 0_{\mathbb{N}}, \mathbf{S}\left(\mathbf{S} 0_{\mathbb{N}}\right), \ldots$ as usual. If the meaning becomes clear from the context, we may drop the subscript $\mathbb{N}$. Moreover, $s \leq t$ is used in the obvious way to denote $s<t \vee s=t$.

$\left\langle t_{1}, \ldots, t_{k}\right\rangle$ denotes for any $k \in \mathbb{N}$ and terms $t_{1}, \ldots, t_{k}$ the result of \langle\rangle$_{k}\left(t_{1}, \ldots, t_{k}\right)$, while \langle\rangle$_{k}$ shall be the $k$-ary function symbol in $\mathcal{L}_{\mathrm{PA}}$ corresponding to one of the the usual primitive recursive injective functions $\mathbb{N}^{k} \rightarrow \mathbb{N}$ for mapping finite lists of natural numbers of length $k$ into the natural numbers.

Usual primitive recursive functions for projection $(m, n) \mapsto(m)_{n}$, list construction $(m, n) \mapsto$ $\operatorname{cons}(m, n)$, list concatenation $(m, n) \mapsto m * n$, and for computing the length $n \mapsto \operatorname{lh}(n)$ of a list shall be used ambiguously to denote the application of its corresponding function symbol in $\mathcal{L}_{\mathrm{PA}}$ to terms. In particular, we have: $\left\langle n_{1}, \ldots, n_{k}\right\rangle=0$ if and only if $k=0$. If $n \neq 0$ holds, then there is exactly one $k \neq 0$ and natural numbers $n_{1}, \ldots, n_{k}$ such that $n=\left\langle n_{1}, \ldots, n_{k}\right\rangle$ holds. For each $i<\operatorname{lh}(n)$, we have $(n)_{i}<n$. For each $0 \leq i \leq k$, we have $\left(\left\langle n_{0}, \ldots, n_{k}\right\rangle\right)_{i}=n_{i}$. We have also $\operatorname{lh}\left(\left\langle n_{1}, \ldots, n_{k}\right\rangle\right)=k, \operatorname{cons}\left(n,\left\langle n_{1}, \ldots, n_{k}\right\rangle\right)=\left\langle n, n_{1}, \ldots, n_{k}\right\rangle$, and $\left\langle n_{1}, \ldots, n_{k}\right\rangle *\left\langle m_{1}, \ldots, m_{l}\right\rangle=\left\langle n_{1}, \ldots, n_{k}, m_{1}, \ldots, m_{l}\right\rangle$.

Convention 4.4. $\mathcal{L}$ will denote in the following either $\mathcal{L}_{\text {PA }}^{2}$ or any extension of $\mathcal{L}_{\mathrm{PA}}$ by new relation symbols. We will introduce common notions for such languages $\mathcal{L}$.

Definition 4.5. $\mathcal{L}$ terms $s, t, r$ are defined as usual inductively from function symbols and number variables. Since in this section $\mathcal{L}$ extends $\mathcal{L}_{\mathrm{PA}}$ only by relation symbols or variables of the second sort, all such terms are $\mathcal{L}_{\mathrm{PA}}$ terms. A constant is a nullary function symbol. If $f$ is an $n$-ary function symbol of $\mathcal{L}_{\mathrm{PA}}$ and $\vec{t}=t_{1}, \ldots, t_{n}$ is a list of terms, then we set $f(\vec{t}):=f\left(t_{1}, \ldots, t_{n}\right):=f \vec{t}:=f t_{1} \ldots t_{n}$ and this holds analogously for lists introduced by the $\bar{t}^{(n)}$ notation. For closed terms $t$, we mean by $t^{\mathbb{N}}$ the numerical value of $t$, i.e., the canonical valuation of $t$ in the standard model $\mathbb{N}$.

Definition 4.6. $\mathcal{L}$ formulas are defined inductively as usual by use of parentheses and the basic logical symbols and we write ambiguously $A \in \mathcal{L}$ to stress that $A$ is an $\mathcal{L}$ formula. For terms $s, t$, we may sometimes write $s \neq t$ for $\neg(s=t)$. Atomic $\mathcal{L}$ formulas are equations $s=t$ and formulas $R t_{1} \ldots t_{n}$, while $R \in \mathcal{L}$ is an $n$-ary relation symbol and $t_{1}, \ldots, t_{n}$ are terms.

For the case that $\mathcal{L}$ is $\mathcal{L}_{\mathrm{PA}}^{2}$, then also $X t$ is an atomic formula for any set variable $X$ and term $t . \mathcal{L}_{\mathrm{PA}}^{2}$ formulas further allow for quantification over set variables and we call an $\mathcal{L}_{\mathrm{PA}}^{2}$ formula arithmetical if it does not contain such a quantification (but set variables may still occur).

For $n$-ary relation symbols or set variables $R$ of $\mathcal{L}$, a formula $A$ is positive in $R$ if it occurs only positively in the usual sense, i.e., no atomic formula of the form $R\left(t_{1}, \ldots, t_{n}\right)$ occurs negated in the formula which is obtained from $A$ by translating first each subformula of the form $B_{1} \rightarrow B_{2}$ to $\neg B_{1} \vee B_{2}$ and where we then move every negation symbol $\neg$ towards atomic formulas, while making use of De Morgan's laws and the law of double negation. ${ }^{5}$

\section{Definition 4.7.}

(a) For any language $\mathcal{L}$ that is $\mathcal{L}_{\mathrm{PA}}^{2}$ or (possibly) extends $\mathcal{L}_{\mathrm{PA}}$ by new relation or function symbols, a standard derivability notion $\vdash$ shall be given that is based on a Hilbert-style

\footnotetext{
${ }^{5}$ Compare this definition of positive formula with the definition of $\mathrm{For}^{+}$in the setting of FIT.
} 
deduction system for classical logic with equality axioms (in the first sort). In particular for $\mathcal{L}_{\text {PA }}^{2}$, we assume besides modus ponens the usual axioms and rules for quantification over set variables.

(b) We write $\vdash A$ for any $A \in \mathcal{L}$ to denote the derivability of $A$ in this logic. Moreover, if $\mathrm{T}$ is a theory (i.e., a collection of non-logical axioms) with language $\mathcal{L}_{\mathrm{T}}$, then writing $\mathrm{T} \vdash A$ for any $A \in \mathcal{L}_{\mathrm{T}}$ denotes the derivability of $A$ from the axioms of $\mathrm{T}$ and this logic. For any set of formulas $\Gamma$, we write $\vdash \Gamma$ and $\mathrm{T} \vdash \Gamma$ in order to denote that $\vdash A$ and $\mathrm{T} \vdash A$ hold, respectively, for each $A \in \Gamma$.

Notation 4.8. For an $n$-ary relation symbol $R$ with $n \geq 1$ and $\vec{t}=t_{1} \ldots t_{n}$, we write $R(\vec{t})$ for $R t_{1} \ldots t_{n}$. and if $n=1$, we also introduce the following notation: $t \in R:=R t$ and $t \notin R:=\neg R t$. Then $(\forall x \in R) A$ and $(\exists x \in R) A$ stand for $\forall x(R(x) \rightarrow A)$ and $\exists x(R(x) \wedge A)$, respectively. These conventions shall hold analogously also for set variables $X$. If $\triangleleft$ is a binary relation symbol, we use expressions $(\forall x \triangleleft t) A$ and $(\exists x \triangleleft t) A$ to abbreviate $\forall x(x \triangleleft t \rightarrow A)$ and $\exists x(x \triangleleft t \wedge A)$, respectively.

Definition 4.9. The first-order theory PA is based on the language $\mathcal{L}_{\mathrm{PA}}$ and its non-logical axioms are the usual axioms of Peano arithmetic with complete induction, while for each relation symbol $R_{f}$ that stems from a function symbol $f$ of arity $n \geq 1$, we have for $\vec{x}=$ $x_{1}, \ldots, x_{n}$ the axiom $\forall \vec{x}\left(R_{f} \vec{x} \leftrightarrow f \vec{x}=0\right)$. There is no non-logical axiom for the unary relation symbol $\mathrm{U}$ (besides in an instance of complete induction).

Definition 4.10. (Arithmetical) operator forms are objects of the form

\section{$\Lambda X . \mathcal{A}$}

for $\mathcal{L}_{\mathrm{PA}}^{2}$ class terms of the form $\mathcal{A}=\Lambda x$.A such that $A$ is an arithmetical formula with $X$ being the only set variable that may occur in it (compare also with Section 2) and $x$ is the only free number variable that may occur in it. ${ }^{6}$ Note that the unary relation symbol $\mathrm{U}$ may occur in $A$. We use $\mathfrak{A}, \mathfrak{B}, \mathfrak{C}, \mathfrak{D}$ as syntactic variables for operator forms. For each $\mathcal{L}$ class terms $\mathcal{B}$, we set $(\Lambda X . \mathcal{A})(\mathcal{B}):=\mathcal{A}(\mathcal{B} / X)$ while note that the expression $\mathcal{A}(\mathcal{B} / X)$ may yield an $\mathcal{L}$ formula here. We write $\mathfrak{A}(R)$ to denote $\mathfrak{A}(\Lambda x . R x)$ when $R$ is a unary relation symbol in $\mathcal{L}$ or a set variable. Positive operator forms are operator forms $\mathfrak{A}:=\Lambda X . \Lambda x$. A where $X$ occurs only positively in $A$.

Notation 4.11. We have the following abbreviations for some formulas and operator forms:

- $\mathrm{Cl}_{\mathfrak{A}}(\mathcal{A}):=\forall x(\mathfrak{A}(\mathcal{A}, x) \rightarrow \mathcal{A}(x))$ for each operator form $\mathfrak{A}$ and $\mathcal{L}$ class term $\mathcal{A}$.

and for a binary relation symbol $\triangleleft$ in $\mathcal{L}_{\mathrm{PA}}$ and any class term $\mathcal{A}$, we also have

- $\mathrm{Acc}_{\triangleleft}:=\Lambda X . \Lambda x . \forall y \triangleleft x(X y)$,

- $\operatorname{Prog}_{\triangleleft}(\mathcal{A}):=\mathrm{Cl}_{\triangleleft}(\mathcal{A}):=\mathrm{Cl}_{\mathrm{Acc} \triangleleft}(\mathcal{A})$,

- $\mathrm{TI}_{\triangleleft}:=\Lambda X . \Lambda x .\left(\operatorname{Prog}_{\triangleleft}(X) \rightarrow \forall y \triangleleft x(X y)\right)$, and

Note that we shall usually write $\operatorname{Prog}_{\triangleleft}$ instead of $\mathrm{Cl}_{\triangleleft}$. If $\triangleleft$ is clear from the context, we may just write Acc, Cl, Prog, and TI instead of $\mathrm{Acc}_{\triangleleft}, \mathrm{Cl}_{\triangleleft}, \mathrm{Prog}_{\triangleleft}$, and $\mathrm{TI}_{\triangleleft}$, respectively.

\footnotetext{
${ }^{6}$ Recall that $\mathfrak{A}:=\Lambda X . \Lambda x . A$ is intended to define an operator $\Phi_{\mathfrak{A}}: \mathcal{P}(\mathbb{N}) \rightarrow \mathcal{P}(\mathbb{N})$ where $\mathfrak{A}(X, x)$ corresponds to " $x \in \Phi_{\mathfrak{A}}(X)$ " for some interpretation $X \subseteq \mathbb{N}$ and $x \in \mathbb{N}$ of $x$ and $X$.
} 


\subsection{The Accessible Part Theory TID}

\section{Definition 4.12.}

(a) For each operator form $\mathfrak{A}$, let $P_{\mathfrak{A}}$ denote a new unary relation symbol not in $\mathcal{L}_{\mathrm{PA}}$. Then, $P_{\triangleleft}$ abbreviates $P_{\mathrm{Acc} \triangleleft}$ for any binary relation symbol $\triangleleft$ in $\mathcal{L}_{\mathrm{PA}}$.

(b) $\mathcal{L}_{\mathrm{TID}}:=\mathcal{L}_{\mathrm{PA}} \cup\left\{P_{\triangleleft}: \triangleleft\right.$ is a binary relation symbol in $\left.\mathcal{L}_{\mathrm{PA}}\right\}$ defines the language of TID.

Definition 4.13. $\operatorname{Pos}_{0}$ denotes the collection of formulas $A \in \mathcal{L}_{\text {TID }}$ such that $P_{\triangleleft}$ occurs at most positively in $A$ for any binary relation symbol $\triangleleft$ in $\mathcal{L}_{\mathrm{PA}}$. Then define $\operatorname{Pos}_{1}(a)$ for any number variable $a$ as the collection of $\mathcal{L}_{\text {TID }}$ formulas $A$ such that one of the following cases holds:

- $A \in \operatorname{Pos}_{0}$ or

- $A=\forall \vec{x}\left(B_{1} \rightarrow B_{2}\right)$ for $a \notin \mathrm{FV}\left(B_{1}\right)$ and $B_{1}, B_{2} \in \operatorname{Pos}_{0}$,

while $\vec{x}$ is a (possibly empty) list of variables. This is motivated by FT from the setting of FIT. We write $\Lambda a . A \in \operatorname{Pos}_{1}$ in order to denote $A \in \operatorname{Pos}_{1}(a) .{ }^{7}$ Let $\mathrm{Neg}_{0}:=\left\{A \in \mathcal{L}_{\mathrm{TID}}: \neg A \in \operatorname{Pos}_{0}\right\}$.

Remark 4.14. The variable condition $a \notin \mathrm{FV}\left(B_{1}\right)$ in the definition of $\operatorname{Pos}_{1}(a)$ is not necessary (see [Ran15, Section 8.3]). We keep it for a more direct relationship between TID and FIT.

Definition 4.15. TID arises from the axioms of PA without complete induction by adding the following axioms and axiom schemes for binary relation symbols $\triangleleft \in \mathcal{L}_{\mathrm{PA}}$ and $\mathcal{B} \in \mathrm{Pos}_{1}$ :

$$
\begin{aligned}
\text { (Ind) } & \mathcal{B}(0) \wedge \forall x(\mathcal{B}(x) \rightarrow \mathcal{B}(\mathbf{S} x)) \rightarrow \forall x \mathcal{B}(x) \\
\text { (Cl) } & \operatorname{Prog}_{\triangleleft}\left(P_{\triangleleft}\right) \\
\text { (TID) } & \operatorname{Prog}_{\triangleleft}(\mathcal{B}) \rightarrow \forall x\left(P_{\triangleleft} x \rightarrow \mathcal{B}(x)\right)
\end{aligned}
$$

where $(\mathrm{Cl})$ is called closure and (TID) is called typed inductive definition.

Remark 4.16. From (Cl) and (TID), we get a fixed-point principle

$$
\text { (FP) } \forall x\left(P_{\triangleleft} x \leftrightarrow \operatorname{Acc}_{\triangleleft}\left(P_{\triangleleft}, x\right)\right)
$$

for each binary relation symbol $\triangleleft$ in $\mathcal{L}_{\mathrm{PA}}$. We write $(\mathrm{Cl})$ ambiguously for $(\mathrm{FP})$.

Remark 4.17. Instead of (Ind), we have also a course-of-value variant of complete induction $\forall x\left(\forall x_{0}<_{\mathbb{N}} x \mathcal{B}\left(x_{0}\right) \rightarrow \mathcal{B}(x)\right) \rightarrow \forall x \mathcal{B}(x)$ at hand for all $\mathcal{B} \in$ Pos $_{1}$ which we shall tacitly use.

\subsection{Embedding TID into FIT}

Theorem 4.18. For each $n$-ary function symbol $f \in \mathcal{L}_{\mathrm{PA}}$, there is a closed $\mathcal{L}_{\mathrm{FIT}}$ term $\mathrm{pr}_{f}$ such that the following holds:

(a) FIT proves the reformulation of every defining equation of $f$ from PA with respect to $\mathrm{pr}_{f}$, while interpreting number variables $x$ as individual variables $x$ with $x \in \mathrm{N}$.

(b) $\mathrm{FIT} \vdash \operatorname{pr}_{f} \in \mathrm{N}^{n} \rightarrow \mathrm{N}$.

\footnotetext{
${ }^{7}$ As an example, let $A:=\forall y\left(P_{\triangleleft} y \rightarrow \forall x \triangleleft a\left(P_{\triangleleft} f x y\right)\right)$ for some binary function symbol $f$ in $\mathcal{L}_{\mathrm{PA}}$ and $a$ a number variable. Then we have $A \in \operatorname{Pos}_{1}(a)$ and $\Lambda a . A \in \operatorname{Pos}_{1}$.
} 
Proof. $\mathrm{pr}_{f}$ can be obtained by making use of fix from Lemma 3.11.(b) and the induction principle (FT-Ind). See [Ran15, Section 6.2] for more details.

Definition 4.19. Based on $\operatorname{pr}_{f}$ from Theorem 4.18, we define for each $\mathcal{L}_{\mathrm{PA}}$ term $t$ the translation $t^{\bullet}$ to an $\mathcal{L}_{\mathrm{FIT}}$ term recursively on the build-up of $t: x$ if $t$ is a variable $x, \mathrm{pr}_{c}$ if $t$ is a constant $c$, and $\operatorname{pr}_{f} t_{1}^{\bullet} \ldots t_{n}^{\bullet}$ if $t$ is $f t_{1} \ldots t_{n}$ with $f \in \mathrm{PR}^{n}$ and $n \geq 1$.

The translation on terms is now extended to $\mathcal{L}_{\text {TID }}$ formulas $A$. We define the $\mathcal{L}_{\text {FIT }}$ formula $A^{\bullet}$ recursively on the build-up of an $\mathcal{L}_{\mathrm{TID}}$ formula $A$ (and where $\mathbb{Q}_{\triangleleft}:=\left\{\langle x, y\rangle:(x \triangleleft y)^{\bullet}\right\}$ ):

$$
\begin{aligned}
& (s=t)^{\bullet}:=\left(s^{\bullet}=t^{\bullet}\right) \quad\left(R_{f} t_{1} \ldots t_{n}\right)^{\bullet}:=\left(\operatorname{pr}_{f} t_{1}^{\bullet} \ldots t_{n}^{\bullet}=0\right) \\
& (\mathrm{U} t)^{\bullet}:=t^{\bullet} \in \mathrm{U} \quad\left(P_{\triangleleft} t\right)^{\bullet}:=t^{\bullet} \in \mathrm{I}_{\mathrm{N}, \mathbb{Q}_{\triangleleft}} \\
& (\neg B)^{\bullet}:=\neg\left(B^{\bullet}\right) \quad(B \circ C)^{\bullet}:=B^{\bullet} \circ C^{\bullet} \quad(\text { for } \circ \in\{\vee, \wedge, \rightarrow\}) \\
& (\forall x B)^{\bullet}:=\forall x\left(x \in \overline{\mathrm{N}} \vee B^{\bullet}\right) \quad(\exists x B)^{\bullet}:=\exists x\left(x \in \mathrm{N} \wedge B^{\bullet}\right)
\end{aligned}
$$

The expression $\left\{\langle x, y\rangle:(x \triangleleft y)^{\bullet}\right\}$ for $\mathbb{Q}_{\triangleleft}$ abbreviates the expression $\left\{z: z=\left\langle(z)_{0},(z)_{1}\right\rangle \wedge(x \triangleleft\right.$ $\left.y)^{\bullet}\left((z)_{0} / x,(z)_{1}\right) / y\right\}$, i.e., $\left\{z: z=\left\langle(z)_{0},(z)_{1}\right\rangle \wedge R_{f}(z)_{0}(z)_{1}\right\}$ where $f$ is such that $\triangleleft$ is $R_{f}$.

Remark 4.20 .

(a) Note that we defined $A^{\bullet}$ in case of $A$ being $\forall x B$ in such a way that we can show that $A \in \mathrm{For}^{+}$implies $A^{\bullet} \in \operatorname{Pos}_{0}$ in Lemma 4.21. If we would have defined it to be $\forall x\left(x \in \mathrm{N} \rightarrow B^{\bullet}\right)$, then apparently $\mathrm{N}$ would occur negatively in $A^{\bullet}$.

(b) It can be readily checked that $A^{\bullet}$ is indeed a $\mathcal{L}_{\mathrm{FIT}}$ formula. Moreover, $A$ and $A^{\bullet}$ have the same free variables. In particular, note that $\left.(x \triangleleft y)^{\bullet}\left((z)_{0} / x,(z)_{1}\right) / y\right\}$ contains only $z$ as a free variable.

(c) We will use the expression $\mathbb{Q}_{\triangleleft}$ without further mentioning in order to denote the type that we introduced in the definition of $\left(P_{\triangleleft} t\right)^{\bullet}$. Recall also that $(x \triangleleft y)^{\bullet}$ equals $\operatorname{pr}_{f} x y=0$ for some binary function symbol $f \in \mathcal{L}_{\mathrm{PA}}$ because the binary relation symbol $\triangleleft \in \mathcal{L}_{\mathrm{PA}}$ is of the form $R_{f}$ for such an $f$.

\section{Lemma 4.21.}

(a) For each $A \in \operatorname{Pos}_{0}$ there exists some $A^{\prime} \in \mathrm{For}^{+}$such that $\mathrm{FV}\left(A^{\bullet}\right)=\mathrm{FV}\left(A^{\prime}\right)$ and FIT $\vdash A^{\bullet} \leftrightarrow A^{\prime}$ hold.

(b) For each $A \in \mathrm{Neg}_{0}$ there exists some $A^{\prime} \in \mathrm{For}^{+}$such that $\mathrm{FV}\left(A^{\bullet}\right)=\mathrm{FV}\left(A^{\prime}\right)$ and FIT $\vdash A^{\bullet} \leftrightarrow \neg A^{\prime}$ hold.

Proof. By simultaneous induction on the build-up of $A$.

Definition 4.22. For every $A \in \mathcal{L}_{\mathrm{TID}}$, we define $A_{\mathrm{N}}^{\bullet}$ as $A^{\bullet}$ if $\mathrm{FV}(A)=\emptyset$ and otherwise as $x_{1} \in \mathrm{N} \rightarrow \ldots \rightarrow x_{n} \in \mathrm{N} \rightarrow A^{\bullet}$, given $\mathrm{FV}(A)=\left\{x_{1}, \ldots, x_{n}\right\}$ for some $n \neq 0$.

Lemma 4.23. For each $B \in \operatorname{Pos}_{1}(a)$, there is an $\mathcal{L}_{\mathrm{FIT}}$-term $t$ and a function type $\mathbb{F} \in \mathrm{FT}$ such that $\mathrm{FIT} \vdash \forall x\left(t x \in \mathbb{F} \leftrightarrow B^{\bullet}(x / a)\right)$ holds.

Proof. We distinguish the following cases on $B \in \operatorname{Pos}_{1}(a)$ :

1. If $B \in \operatorname{Pos}_{0}$, then Lemma 4.21 provides some $B^{\prime} \in$ For $^{+}$such that FIT $\vdash B^{\bullet} \leftrightarrow B^{\prime}$ holds, so for $\mathbb{F}:=\left\{a: B^{\prime}\right\}$ we have $\mathbb{F} \in \mathrm{FT}$. Moreover, with $t:=\lambda x . x$, we get the claim. 
2. If $B$ is of the form $\forall \vec{y}\left(B_{1} \rightarrow B_{2}\right)$ with $a \notin \mathrm{FV}\left(B_{1}\right), \vec{y}=y_{1}, \ldots, y_{n}$, and $B_{1}, B_{2} \in \mathrm{Pos}_{0}$, we first get $B_{1}^{\prime}, B_{2}^{\prime} \in$ For $^{+}$from Lemma 4.21 such that $B_{i}^{\bullet} \leftrightarrow B_{i}^{\prime}$ and $\mathrm{FV}\left(B_{i}^{\bullet}\right)=\mathrm{FV}\left(B_{i}^{\prime}\right)$ holds for $i=1,2$. Then we set $\mathbb{Q}_{1}:=\left\{z: z=\left\langle(z)_{0}, \ldots,(z)_{n-1}\right\rangle \wedge B_{1}^{\prime}\left((z)_{0} / y_{1}, \ldots,(z)_{n-1} / y_{n}\right)\right\}$, $\mathbb{Q}_{2}:=\left\{z: z=\left\langle(z)_{0}, \ldots,(z)_{n}\right\rangle \wedge B_{2}^{\prime}\left((z)_{n} / a,(z)_{0} / y_{1}, \ldots,(z)_{n-1} / y_{n}\right)\right\}, \mathbb{F}:=\mathbb{Q}_{1} \rightarrow \mathbb{Q}_{2}$, and $t:=\lambda x, z \cdot\left\langle x,(z)_{0}, \ldots,(z)_{n-1}\right\rangle$. Obviously $\mathbb{F} \in \mathrm{FT}$ holds and then similar as in [Fef92, 6.3], we have over FIT and for any $x$ that $t x \in \mathbb{F}$ is equivalent to $\forall z\left(z \in \mathbb{Q}_{1} \rightarrow t x z \in \mathbb{Q}_{2}\right)$ which is equivalent to $\forall \vec{y}\left(B_{1}^{\bullet} \rightarrow t x\left\langle y_{1}, \ldots, y_{n}\right\rangle \in \mathbb{Q}_{2}\right)$ which is equivalent to $\forall \vec{y}\left(B_{1}^{\bullet} \rightarrow\left\langle x, y_{1}, \ldots, y_{n}\right\rangle \in\right.$ $\left.\mathbb{Q}_{2}\right)$ which is equivalent to $\forall \vec{y}\left(B_{1}^{\bullet} \rightarrow B_{2}^{\bullet}(x / a)\right)$ which is equivalent to $B^{\bullet}(x / a)$. Hence, we get the claim. Note that $n=0$ is possible, so $\forall \vec{y}\left(B_{1}^{\bullet} \rightarrow t x\left\langle y_{1}, \ldots, y_{n}\right\rangle \in \mathbb{Q}_{2}\right)$ denotes then $B_{1}^{\bullet} \rightarrow\left(t x\langle\rangle \in \mathbb{Q}_{2}\right)$.

Theorem 4.24. If $A$ is an instance of (Ind), (Cl), or (TID), then we have FIT $\vdash A^{\bullet}$.

Proof. Let $A$ be an instance of (Ind), (Cl), or (TID). We have to show FIT $\vdash A^{\bullet}$.

1. For $(\mathrm{Cl})$ : If $A=\operatorname{Prog}_{\triangleleft}\left(P_{\triangleleft}\right)$ holds for some $\triangleleft$, then we have that $A^{\bullet}$ is logically equivalent over FIT to $\mathrm{Cl}_{\mathrm{N}, \mathbb{Q}_{\triangleleft}}\left(\Lambda z . z \in \mathrm{I}_{\mathrm{N}, \mathbb{Q}_{\triangleleft}}\right)$, and this is an instance of $(\mathrm{FT}-\mathrm{Cl})$. We have over FIT:

$$
\begin{aligned}
& \left(\operatorname{Prog}_{\triangleleft}\left(P_{\triangleleft}\right)\right)^{\bullet} \\
& \leftrightarrow\left(\forall x\left(\operatorname{Acc}_{\triangleleft}\left(P_{\triangleleft}, x\right) \rightarrow P_{\triangleleft} x\right)\right)^{\bullet} \\
& \leftrightarrow \forall x\left(x \in \overline{\mathrm{N}} \vee\left(\left(\operatorname{Acc}_{\triangleleft}\left(P_{\triangleleft}, x\right)\right)^{\bullet} \rightarrow x \in \mathrm{I}_{\mathrm{N}, \mathbb{Q}_{\triangleleft}}\right)\right) \\
& \leftrightarrow \forall x\left(x \in \overline{\mathrm{N}} \vee\left(\forall y\left(y \in \overline{\mathrm{N}} \vee\left((y \triangleleft x)^{\bullet} \rightarrow y \in \mathrm{I}_{\mathrm{N}, \mathbb{Q}_{\triangleleft}}\right)\right) \rightarrow x \in \mathrm{I}_{\mathrm{N}, \mathbb{Q}_{\triangleleft}}\right)\right) \\
& \leftrightarrow \forall x\left(x \in \overline{\mathrm{N}} \vee\left(\forall y\left(y \in \overline{\mathrm{N}} \vee\left(\langle y, x\rangle \in \mathbb{Q}_{\triangleleft} \rightarrow y \in \mathrm{I}_{\mathrm{N}, \mathbb{Q}_{\triangleleft}}\right)\right) \rightarrow x \in \mathrm{I}_{\mathrm{N}, \mathbb{Q}_{\triangleleft}}\right)\right) \\
& \leftrightarrow(\forall x \in \mathrm{N})\left((\forall y \in \mathrm{N})\left(\langle y, x\rangle \in \mathbb{Q}_{\triangleleft} \rightarrow y \in \mathrm{I}_{\mathrm{N}, \mathbb{Q}_{\triangleleft}}\right) \rightarrow x \in \mathrm{I}_{\mathrm{N}, \mathbb{Q}_{\triangleleft}}\right) \\
& \leftrightarrow \mathrm{Cl}_{\mathrm{N}, \mathbb{Q}_{\triangleleft}}\left(\Lambda z . z \in \mathrm{I}_{\mathrm{N}, \mathbb{Q}_{\triangleleft}}\right)
\end{aligned}
$$

2. For (Ind) and (TID): Let $B \in \operatorname{Pos}_{1}(a)$ be arbitrary. By Lemma 4.23 some $\mathcal{L}_{\text {FIT }}$-term $t$ and function type $\mathbb{F} \in \mathrm{FT}$ exist such that we have

$$
\text { FIT } \vdash \forall x\left(t x \in \mathbb{F} \leftrightarrow B^{\bullet}(x / a)\right)
$$

2.1. If $A=\mathcal{B}(0) \wedge \forall x(\mathcal{B}(x) \rightarrow \mathcal{B}(\mathbf{S} x)) \rightarrow \forall x \mathcal{B}(x)$ holds for $\mathcal{B}=\Lambda a$. B: We note that for $B_{1}:=B(a / \mathbf{S} a)$ one can prove (by induction on the build-up of $B$ ) that $B_{1}^{\bullet}$ is $B^{\bullet}\left(a / \mathrm{s}_{\mathbf{N}} a\right)$. So, with $\mathcal{B}(\mathbf{S} x)^{\bullet}$ being $(B(a / \mathbf{S} x))^{\bullet}$ this becomes $\left(B_{1}(x)\right)^{\bullet}$, i.e., we get $B_{1}^{\bullet}(a / x)$ and hence $\left(B^{\bullet}\left(a / \mathrm{s}_{\mathrm{N}} a\right)\right)(a / x)$. So, we obtain that $\mathcal{B}(\mathbf{S} x)^{\bullet}$ is $B^{\bullet}\left(a / \mathrm{s}_{\mathrm{N}} x\right)$, while note that for any $B^{\prime} \in \mathcal{L}_{\mathrm{TID}}$, we have that $B^{\prime}$ and $B^{\prime \bullet}$ share the same first-order variables. For proving $A^{\bullet}$, we can therefore assume that

$$
\begin{aligned}
& B^{\bullet}(0 / a) \\
& \forall x\left(x \in \overline{\mathrm{N}} \vee\left(B^{\bullet}(x / a) \rightarrow B^{\bullet}\left(\mathrm{s}_{\mathrm{N}} x / a\right)\right)\right)
\end{aligned}
$$

holds, and we have to show $\forall x\left(x \in \overline{\mathrm{N}} \vee B^{\bullet}(x / a)\right)$, while this is equivalent to $t \in \mathrm{N} \rightarrow \mathbb{F}$ due to (1). Now we can directly apply (FT-Ind) because (2) is equivalent to $t 0 \in \mathbb{F}$ and (3) is equivalent to $(\forall x \in \mathrm{N})\left(t x \in \mathbb{F} \rightarrow t\left(\mathrm{~s}_{\mathrm{N}}\right) \in \mathbb{F}\right)$.

2.2. If $A=\operatorname{Prog}_{\triangleleft}(\mathcal{B}) \rightarrow \forall x\left(P_{\triangleleft} x \rightarrow \mathcal{B}(x)\right)$ holds for $\mathcal{B}=\Lambda a$.B: With $\left(\operatorname{Prog}_{\triangleleft}(\mathcal{B})\right)^{\bullet}$ being $(\forall x \in \mathrm{N})(\forall y \in \mathrm{N})\left(\langle y, x\rangle \in \mathbb{Q}_{\triangleleft} \rightarrow(\mathcal{B}(y))^{\bullet} \rightarrow(\mathcal{B}(x))^{\bullet}\right)$, we get that FIT proves the following:

$$
\left.\begin{array}{rl}
\left(\operatorname{Prog}_{\triangleleft}(\mathcal{B})\right)^{\bullet} & \leftrightarrow(\forall x \in \mathrm{N})(\forall y \in \mathrm{N})\left(\langle y, x\rangle \in \mathbb{Q}_{\triangleleft} \rightarrow t y \in \mathbb{F} \rightarrow t x \in \mathbb{F}\right) \\
& \leftrightarrow \mathrm{Cl}_{\mathrm{N}, \mathbb{Q}_{\triangleleft}}(\Lambda z . t z \in \mathbb{F})
\end{array}\right\}
$$


This accumulates in the provability of $A^{\bullet}$. Namely, assume $\left(\operatorname{Prog}_{\triangleleft}(\mathcal{B})\right)^{\bullet}$ and get $t \in\left(\mathrm{I}_{\mathbb{N}, \mathbb{Q}_{\triangleleft}} \rightarrow\right.$ $\mathbb{F}$ ) from (4) and (FT-ID), hence (1) yields that $\forall x\left(x \in \mathrm{I}_{\mathrm{N}, \mathbb{Q}_{\triangleleft}} \rightarrow t x \in \mathbb{F}\right)$ is equivalent to $\forall x\left(x \in \mathrm{I}_{\mathbb{N}, \mathbb{Q}_{\triangleleft}} \rightarrow B^{\bullet}(x / a)\right)$. The latter implies $(\forall x \in \mathrm{N})\left(x \in \mathrm{I}_{\mathbf{N}, \mathbb{Q}_{\triangleleft}} \rightarrow B^{\bullet}(x / a)\right)$, so we are done because $\left(\forall x\left(P_{\triangleleft} x \rightarrow \mathcal{B}(x)\right)\right)^{\bullet}$ is $(\forall x \in \mathrm{N})\left(x \in \mathrm{I}_{\mathrm{N}, \mathbb{Q}_{\triangleleft}} \rightarrow B^{\bullet}(x / a)\right)$.

Corollary 4.25. TID $\vdash A$ implies $\mathrm{FIT} \vdash A_{\mathrm{N}}^{\bullet}$ for each $A \in \mathcal{L}_{\mathrm{TID}}$.

Proof. The claim follows essentially from Theorems 4.18 and 4.24. In particular, we remark that for FIT the propositional logical rules and axioms and the quantificational logic for individual variables correspond (under the translation of Definition 4.19) to first-order predicate logic in the setting of TID.

\section{Ordinals}

The lower bounds for the proof-theoretic ordinal of both FIT and TID are determined in

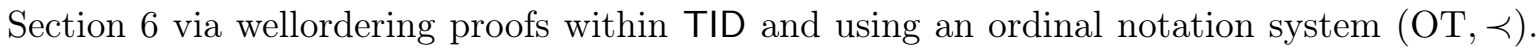
For carrying out these wellordering proofs in all details, we would need to provide a full definition of $(\mathrm{OT}, \prec)$ and verify that all needed properties are provable within TID. Due to constraints on the length of this article, though, we refer the interested reader to [Ran15, Chapter 3] for further details and provide in the appendix hints on the definition and properties of $(\mathrm{OT}, \prec)$.

The ordinal notation system $(\mathrm{OT}, \prec)$ is based on a framework for representing ordinals that we shall introduce in this section. We shift to the literature most of the preparatory work that is needed to formulate the ordinals that are involved here and explain only as much as to make this article sufficiently self-contained and provide a structural impression on which properties we demand from $(\mathrm{OT}, \prec)$. In particular, we use [Sch54] and the treatment of Klammersymbols (that extends the concept of finitary Veblen functions to the transfinite) as the main source for the finitary Veblen functions because it is the most elaborated source for representation and recursion properties.

\subsection{Ordinal Notations}

Working in the broad set-theoretic framework of Zermelo-Fraenkel set theory ZFC with the axiom of choice, let On denote the class of ordinals and use $\alpha, \beta, \gamma, \delta, \ldots$ (i.e., small Greek letters in general) to denote elements of On. By Lim, we denote the class of limit ordinals, while $\omega$ denotes the first limit ordinal. Moreover, we write 0 for $\emptyset, \alpha<$ on $\beta$ (or just $\alpha<\beta$ ) for $\alpha \in \beta$, and $\alpha \leq$ On $\beta$ (or just $\alpha \leq \beta$ ) for $\alpha \subseteq \beta$. For $\alpha>0$, we let $\Omega_{\alpha}$ denote $\aleph_{\alpha}$, i.e., $\left\{\Omega_{\alpha}: \alpha \in \mathrm{On}\right\}$ is the class of all uncountable initial ordinals, and we write $\Omega$ for $\Omega_{1}$ and $\Omega_{0}$ for 0 . Over ZFC, we have that $\Omega_{\alpha+1}$ is regular. A normal function is a (with respect to $<$ ) strictly increasing continuous function $f$ : On $\rightarrow$ On. Demanding a knowledge about this broad set-theoretic framework, we use common notions and well-known properties of those tacitly, e.g.,

- the notion of $c l u b$ classes $C$ with $C \subseteq$ On and its correspondence to normal functions ${ }^{8}$,

- the existence of the derivative $\operatorname{fix}(f):=\{\alpha \in$ On: $f(\alpha)=\alpha\}$ of a normal function $f$, being a club class itself,

\footnotetext{
${ }^{8}$ Each club class $C$ induces a normal function enum $C$ that enumerates the elements of $C$ in increasing order.
} 
- basic ordinal arithmetic for $\alpha, \beta \in$ On with (ordinal) addition $\alpha+$ On $\beta$ (or just $\alpha+\beta$ ), (ordinal) multiplication $\alpha \cdot$ on $\beta$ (or just $\alpha \cdot \beta$ or $\alpha \beta$ ), and (ordinal) exponentiation $\exp _{\text {On }}(\alpha, \beta)$ (or just $\alpha^{\beta}$ ),

- the usual representation of natural numbers as von Neumann ordinals $(0)_{\text {On }}:=\emptyset$ and $(n+1)_{\text {On }}:=\left\{(n)_{\text {On }}\right\} \cup(n)_{\text {On }}$ for each $n \in \mathbb{N}$ within On, while we identify $(n)_{\text {On }}$ with $n$.

See [Buc16] for more details on the relationship between different approaches to ordinal notations. It shall be clear from the context whether $<$ means $<_{\mathbb{N}}$ or $<_{\text {On }}$ (and similar for the other mentioned expressions).

Definition 5.1. Let $\mathbf{P}:=\left\{\omega^{\eta}: \eta \in \mathrm{On}\right\}$ denote the class of additive principal numbers.

Remark 5.2. For $\alpha \in \mathbf{P}$ and $\beta, \gamma<\alpha$, we have $\beta+\gamma<\alpha$ and $\beta+\alpha=\alpha$.

\section{The finitary Veblen functions}

Definition 5.3. The $n+1$-ary Veblen function $\varphi_{n+1}: \mathrm{On}^{n+1} \rightarrow$ On is obtained for each $n \in \mathbb{N}$ from the $\omega$-exponential function and the binary Veblen function $\varphi_{2}$ by generalizing its definition principle, i.e., we let $\varphi_{1}(\gamma):=\omega^{\gamma}$ for each $\gamma \in$ On and define $\varphi_{n+2}$ for $n \geq 0$ as follows:

- $\varphi_{n+2}\left(0, \bar{\alpha}^{(n)}, \gamma\right):=\varphi_{n+1}\left(\bar{\alpha}^{(n)}, \gamma\right)$.

- If $\alpha_{1}, \alpha_{k}>0$ holds for some $1 \leq k \leq n+1$ with $\alpha_{k+1}=\cdots=\alpha_{n+1}=0$, then $\varphi_{n+2}\left(\bar{\alpha}^{(n+1)}, \gamma\right)$ denotes the $\gamma$-th common fixed-point of the functions

$$
\xi \mapsto \varphi_{n+2}\left(\bar{\alpha}^{(k-1)}, \beta, \xi, \overline{0}^{(n-k+1)}\right)
$$

that are defined on On and for each $\beta<\alpha_{k}$.

Definition 5.4. The small Veblen ordinal is the least ordinal $\alpha>0$ not expressible from ordinals smaller than $\alpha$ and by means of ordinal addition and the finitary Veblen functions.

Notation 5.5. We often write $\varphi$ instead of $\varphi_{n+1}$ if the arity $n+1$ is clear from the context.

Remark 5.6. We have $\Gamma_{0}=\varphi(1,0,0)$ (the Feferman-Schütte ordinal) and $\varepsilon_{0}=\varphi(0,1,0)=$ $\varphi(1,0)$.

Lemma 5.7. Let $k, l \in \mathbb{N}$ and $\alpha_{1}, \ldots, \alpha_{k} \in \mathrm{On}$ be given with $\alpha_{1} \neq 0$ and $\alpha_{k} \neq 0$. Then $\beta<\alpha_{k}$ and $\xi=\varphi\left(\bar{\alpha}^{(k)}, \overline{0}^{(l+1)}\right)$ imply $\varphi\left(\bar{\alpha}^{(k-1)}, \beta, \overline{0}^{(i)}, \xi, \overline{0}^{(j)}\right)=\xi$ for every $\beta, \xi$ and $i, j \in \mathbb{N}$ with $i+j=l$.

Proof. This follows easily from Definition 5.3.

\section{Klammersymbols}

The following introduction to Klammersymbols and related results are included here because Klammersymbols provide the most elaborated source to derive the major properties of the finitary Veblen functions. 
Definition 5.8. Following [Sch54], we introduce Schütte's Klammersymbols ${ }^{9}$ which are a generalization of the finitary Veblen functions to the transfinite by allowing arguments to be indexed by ordinals. A Klammersymbol $K$ is an expression of the form

$$
\left(\begin{array}{lll}
\alpha_{1} & \cdots & \alpha_{n+1} \\
\beta_{1} & \cdots & \beta_{n+1}
\end{array}\right)
$$

for $\alpha_{1}, \ldots, \alpha_{n+1}, \beta_{1}, \ldots, \beta_{n+1} \in \mathrm{On}$ and with the condition

$$
0 \leq \beta_{1}<\beta_{2}<\cdots<\beta_{n+1}
$$

We use $K$ as a syntactic variable to denote a Klammersymbol. Two Klammersymbols $K_{1}$ and $K_{2}$ are defined to be equal, denoted $K_{1}=K_{2}$, in case $K_{1}$ and $K_{2}$ can be transformed into the same Klammersymbol by adding or dropping of columns of the form ${ }_{\beta}^{0}$. We write $K_{1} \neq K_{1}$ in case that $K_{1}$ and $K_{2}$ are not equal.

Given a normal function $f:$ On $\rightarrow$ On such that $f(0)>0$, the value $f K$ of a Klammersymbol $K$ (under $f$ ) is defined as follows:

1. If $K$ is $\left(\begin{array}{l}\gamma \\ 0\end{array}\right)$, then $f K$ is $f(\gamma)$.

2. If $K$ is $\left(\begin{array}{cccc}\gamma & \alpha_{1} & \ldots & \alpha_{n+1} \\ 0 & \beta_{1} & \ldots & \beta_{n+1}\end{array}\right)$ and $\alpha_{i}=0$ for some $i \in\{1, \ldots, n+1\}$, then $f K$ is $f K^{\prime}$ where $K^{\prime}$ is obtained from $K$ by deleting the column $\underset{\beta_{i}}{0}$.

3. If $K$ is $\left(\begin{array}{cccc}\gamma & \alpha_{1} & \ldots & \alpha_{n+1} \\ 0 & \beta_{1} & \ldots & \beta_{n+1}\end{array}\right)$ and $\alpha_{i} \neq 0$ for all $i \in\{1, \ldots, n+1\}$, then $f K$ is the $\gamma$-th common solution $\eta$ for the following equations and for all $\alpha^{\prime}<\alpha_{1}$ and $\beta^{\prime}<\beta_{1}$ :

$$
f\left(\begin{array}{ccccc}
\eta & \alpha^{\prime} & \alpha_{2} & \ldots & \alpha_{n+1} \\
\beta^{\prime} & \beta_{1} & \beta_{2} & \ldots & \beta_{n+1}
\end{array}\right)=\eta
$$

In case of $n=0$, we read $f\left(\begin{array}{ccccc}\eta & \alpha^{\prime} & \alpha_{2} & \ldots & \alpha_{n+1} \\ \beta^{\prime} & \beta_{1} & \beta_{2} & \ldots & \beta_{n+1}\end{array}\right)$ as $f\left(\begin{array}{cc}\eta & \alpha^{\prime} \\ \beta^{\prime} & \beta_{1}\end{array}\right)$.

Definition 5.9. The large Veblen ordinal is the least ordinal $\alpha>0$ not expressible from ordinals smaller than $\alpha$ and by means of ordinal addition and the use of the value of Klammersymbols under the $\omega$-exponential function $\varphi_{1} \cdot{ }^{10}$

Remark 5.10. For all Klammersymbols $K_{1}$ and $K_{2}$, there are ordinals $\gamma_{1}, \ldots, \gamma_{n+1}$ with $\gamma_{1}<$ $\cdots<\gamma_{n+1}$ and ordinals $\alpha_{1}, \ldots, \alpha_{n+1}, \beta_{1}, \ldots, \beta_{n+1}$ such that $K_{1}=\left(\begin{array}{ccc}\alpha_{1} & \ldots & \alpha_{n+1} \\ \gamma_{1} & \ldots & \gamma_{n+1}\end{array}\right)$ and $K_{2}=$ $\left(\begin{array}{lll}\beta_{1} & \ldots & \beta_{n+1} \\ \gamma_{1} & \ldots & \gamma_{n+1}\end{array}\right)$, simply by adding or removing of columns of the form ${ }_{\gamma_{i}}^{0}$ where necessary.

Definition 5.11. A lexicographic order $<$ on Klammersymbols is defined for Klammersymbols $K_{1}$ and $K_{2}$ with $K_{1} \neq K_{2}$ as follows:

1. If $K_{1}=\left(\begin{array}{ccc}\alpha_{1} & \ldots & \alpha_{n+1} \\ \gamma_{1} & \ldots & \gamma_{n+1}\end{array}\right)$ and $K_{2}=\left(\begin{array}{ccc}\beta_{1} & \ldots & \beta_{n+1} \\ \gamma_{1} & \ldots & \gamma_{n+1}\end{array}\right)$ and $i \in\{1, \ldots, n+1\}$ is the largest index with $\alpha_{i} \neq \beta_{i}$, then we have $K_{1}<K_{2}$ in case of $\alpha_{i}<\beta_{i}$ and $K_{2}<K_{1}$ otherwise.

2. If $K_{1}=K_{1}^{\prime}, K_{2}=K_{2}^{\prime}$, and $K_{1}<K_{2}$, then also $K_{1}^{\prime}<K_{2}^{\prime}$.

Proposition 5.12. Let $f:$ On $\rightarrow$ On, $\xi \mapsto \omega^{\xi}$. Then $\xi \mapsto f\left(\begin{array}{l}1 \\ \xi\end{array}\right)$ is a normal function. In particular, we have $f\left(\begin{array}{l}1 \\ \lambda\end{array}\right)=\sup _{\xi<\lambda} f\left(\begin{array}{l}1 \\ \xi\end{array}\right)$ for each $\lambda \in \operatorname{Lim}$.

\footnotetext{
${ }^{9}$ Klammer is the German word for bracket, so Klammersymbols can be read as "bracket symbols".

${ }^{10}$ In Section 8, the conclusion of this article, we shall mention a theory $\operatorname{TID}_{1}^{+}$which has the large Veblen ordinal as its proof-theoretic ordinal.
} 
Proof. See [Sch54, (4.1)-(4.3)].

Proposition 5.13. Let $f: \mathrm{On} \rightarrow \mathrm{On}, \xi \mapsto \omega^{\xi}$ and $K:=\left(\begin{array}{ccc}\alpha_{1} & \ldots & \alpha_{n+1} \\ \gamma_{1} & \ldots & \gamma_{n+1}\end{array}\right)$ be a Klammersymbol. For each Klammersymbol $K^{\prime}$ with $K<K^{\prime}$, the following holds:

(a) $f K=f K^{\prime}$ holds if and only if $k \in\{1, \ldots, n+1\}$ exists such that $\alpha_{k}=f K^{\prime}$ and the following holds:

- $\alpha_{i}=0$ for each $i$ with $1 \leq i<k$, and

- $\alpha_{i}<f K^{\prime}$ for each $i$ with $k<i \leq n+1$.

(b) $f K<f K^{\prime}$ holds if $\alpha_{i}<f K^{\prime}$ holds for all $i \in\{1, \ldots, n+1\}$.

(c) $f K^{\prime}<f K$ holds if

- either $f K^{\prime}<\alpha_{k}$ holds for some $k \in\{1, \ldots, n+1\}$, or

- $n \geq 1$ and $j, k \in\{1, \ldots, n+1\}$ exist such that $j<k, \alpha_{j} \neq 0$, and $\alpha_{k}=f K^{\prime}$.

Proof. See (7.1)-(7.4) in [Sch54]. Note that the negation of the condition given in (a) yields the conditions stated in (b) and (c). For this, note in particular that $\alpha_{k}=f K^{\prime}$ implies $\alpha_{k} \neq 0$ and hence if $\alpha_{i}=0$ holds for each $i$ with $1 \leq i<k$ and the condition of (a) does not hold, then $k<n+1$ holds and $i$ exists with $\alpha_{i} \geq f K^{\prime}$ and $k<i \leq n+1$, leading to one of the conditions in (c).

Lemma 5.14. Let $f:$ On $\rightarrow$ On, $\xi \mapsto \omega^{\xi}$. Then we have

$$
\varphi\left(\alpha_{1}, \ldots, \alpha_{n+1}\right)=f\left(\begin{array}{cccc}
\alpha_{n+1} & \alpha_{n} & \ldots & \alpha_{1} \\
0 & 1 & \ldots & n
\end{array}\right)
$$

where $0,1, \ldots, n$ in the Klammersymbol's second row denote finite ordinals.

Proof. If $n=0$ or $\alpha_{1}=\cdots=\alpha_{n+1}=0$ holds, then the claim is clear. Otherwise, assume $n \neq 0$ and without loss of generality that $\alpha_{1} \neq 0$ holds. Further, let $k \in\{1, \ldots, n\}$ with $\alpha_{k} \neq 0$ and $\alpha_{k+1}=\cdots=\alpha_{n}=0$. The claim now follows by transfinite induction on $\alpha_{k}$ since $\varphi\left(\bar{\alpha}^{(n+1)}\right)$ is the $\alpha_{n+1}$-th common fixed point of the functions

$$
\xi \mapsto \varphi\left(\bar{\alpha}^{(k-1)}, \beta, \xi, \overline{0}^{(n-k)}\right)
$$

given for each $\beta<\alpha_{k}$. Now, we get

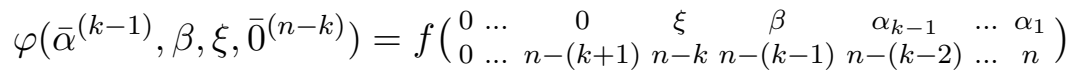

$$
\begin{aligned}
& =f\left(\begin{array}{ccccc}
\xi & \beta & \alpha_{k-1} & \ldots & \alpha_{1} \\
n-k & n-(k-1) & n-(k-2) & \ldots & n
\end{array}\right)
\end{aligned}
$$

from the induction hypothesis and for each $\xi \in$ On. Hence the claim follows from Definition 5.8 and Lemma 5.7.

Corollary 5.15. Let $n \geq 1$ and ordinals $\alpha_{1}, \ldots, \alpha_{n}$ be given, then we have the following:

(a) $\alpha_{i} \leq \varphi\left(\bar{\alpha}^{(n)}\right)$ for all $i \in\{1, \ldots, n\}$.

(b) If $\alpha_{k} \neq 0$ for some $k \in\{1, \ldots, n\}$, then $\alpha_{i}<\varphi\left(\bar{\alpha}^{(n)}\right)$ holds for all $i \in\{1, \ldots, k-1\}$.

Proof. This follows from (3.3) and (6.1) in [Sch54] by making use of Lemma 5.14. 
Corollary 5.16. Let $n \geq 1$ and ordinals $\alpha_{1}, \ldots, \alpha_{n}, \beta_{1}, \ldots, \beta_{n}$ be given. Then $\varphi\left(\bar{\alpha}^{(n)}\right)<$ $\varphi\left(\bar{\beta}^{(n)}\right)$ holds if and only if some $r \in\{1, \ldots, n\}$ exists such that $\alpha_{r} \neq \beta_{r}$ holds with $\alpha_{i}=\beta_{i}$ for all $i \in\{1, \ldots, r-1\}$, and such that one of the following holds:

1. $\alpha_{r}<\beta_{r}$ and $\alpha_{i}<\varphi\left(\bar{\beta}^{(n)}\right)$ for all $i \in\{r+1, \ldots, n\}$, or

2. $\beta_{r}<\alpha_{r}$ and

- either $\varphi\left(\bar{\alpha}^{(n)}\right)<\beta_{k}$ holds for some $k \in\{1, \ldots, n\}$, or

- $\varphi\left(\bar{\alpha}^{(n)}\right)=\beta_{k}$ and $\beta_{i} \neq 0$ for some $i, k \in\{1, \ldots, n\}$ with $k<i$.

Proof. This follows immediately from Proposition 5.13 and Lemma 5.14. For the first case $\alpha_{r}<\beta_{r}$, note that $\alpha_{i}<\varphi\left(\bar{\beta}^{(n)}\right)$ holds anyway for $i \in\{1, \ldots, r\}$ by Corollary 5.15: On the one hand, we have $\beta_{r} \leq \varphi\left(\bar{\beta}^{(n)}\right)$ and so $\alpha_{r}<\varphi\left(\bar{\beta}^{(n)}\right)$, and on the other hand, $\alpha_{r}<\beta_{r}$ also implies $\beta_{r} \neq 0$ which by Corollary 5.15.(b) gives $\alpha_{i}=\beta_{i}<\varphi\left(\bar{\beta}^{(n)}\right)$ for $i \in\{1, \ldots, r-1\}$.

Definition 5.17. Let $n \geq 1$.

(a) $\mathrm{NF}_{n}^{\varphi}\left(\bar{\alpha}^{(n)}\right)$ holds if and only if we have $\alpha_{i}<\varphi\left(\bar{\alpha}^{(n)}\right)$ for each $1 \leq i \leq n$. We read $\mathrm{NF}_{n}^{\varphi}\left(\bar{\alpha}^{(n)}\right)$ as " $\alpha_{1}, \ldots, \alpha_{n}$ is in normal form with respect to $\varphi_{n}$ ".

(b) $\beta={ }_{\mathrm{NF}} \varphi\left(\bar{\alpha}^{(n)}\right)$ denotes $\beta=\varphi\left(\bar{\alpha}^{(n)}\right)$ and $\mathrm{NF}_{n}^{\varphi}\left(\bar{\alpha}^{(n)}\right)$.

Remark 5.18. The following lemma describes an important recursion property that is needed for the definition of the ordinal notation system $(\mathrm{OT}, \prec)$ in [Ran15, Chapter 3]. It explains how to obtain an expression $\varphi_{n}\left(\bar{\beta}^{(n)}\right)$ with $\operatorname{NF}_{n}^{\varphi}\left(\bar{\beta}^{(n)}\right)$, i.e., such that $\beta_{i} \neq \varphi_{n}\left(\bar{\beta}^{(n)}\right)$ holds for each $1 \leq i \leq n$.

Lemma 5.19. Let $n \geq 1, k \in\{1, \ldots, n\}$, and ordinals $\beta_{1}, \ldots, \beta_{n}, \alpha_{1}, \ldots, \alpha_{n}$ be given with $\beta_{k}={ }_{\mathrm{NF}} \varphi\left(\bar{\alpha}^{(n)}\right)$ and $\beta_{k+1}=\cdots=\beta_{n}=0$. Then $\operatorname{NF}_{n}^{\varphi}\left(\bar{\beta}^{(n)}\right)$ holds if and only if $\alpha_{r} \neq \beta_{r}$ holds for some $r \in\{1, \ldots, k\}$ with $\alpha_{i}=\beta_{i}$ for all $i \in\{1, \ldots, r-1\}$ and one of the following holds:

1. $\alpha_{r}<\beta_{r}$, or

2. $\beta_{r}<\alpha_{r}$ and for some $i \in\{1, \ldots, k-1\}$, we have $\beta_{k} \leq \beta_{i}$.

We then get $i \in\{r+1, \ldots, k-1\}$.

Proof. Note that we have $\beta_{k} \neq 0$ due to $\varphi\left(\bar{\alpha}^{(n)}\right) \neq 0$ and our assumption $\beta_{k}={ }_{\mathrm{NF}} \varphi\left(\bar{\alpha}^{(n)}\right)$, and hence by Corollary 5.15 and $\operatorname{NF}_{n}^{\varphi}\left(\bar{a}^{(n)}\right)$, we have

$$
\alpha_{i}<\beta_{k} \leq \varphi\left(\bar{\beta}^{(n)}\right)
$$

for all $i \in\{1, \ldots, n\}$ and also $\beta_{i}<\varphi\left(\bar{\beta}^{(n)}\right)$ for all $i \in\{1, \ldots, k-1\}$. Furthermore, there has to be some $r \in\{1, \ldots, k\}$ such that $\alpha_{r} \neq \beta_{r}$ and $\alpha_{i}=\beta_{i}$ holds for all $i \in\{1, \ldots, r-1\}$ since we have $\alpha_{k}<\beta_{k}$. Recalling the assumption $\beta_{k+1}=\cdots=\beta_{n}=0$, we thus have that $\operatorname{NF}_{n}^{\varphi}\left(\bar{\beta}^{(n)}\right)$ holds if and only if $\beta_{k}<\varphi\left(\bar{\beta}^{(n)}\right)$ holds, i.e., $\varphi\left(\bar{\alpha}^{(n)}\right)<\varphi\left(\bar{\beta}^{(n)}\right)$. Due to Corollary 5.16, it suffices to treat the following three situations:

1. Case $\alpha_{r}<\beta_{r}$ : We have $\alpha_{i}<\varphi\left(\bar{\beta}^{(n)}\right)$ for each $i \in\{r+1, \ldots, n\}$ due to (6) and can use Corollary 5.16 directly to finish the proof for this case.

2. Case $\beta_{r}<\alpha_{r}$ and $\varphi\left(\bar{\alpha}^{(n)}\right)<\beta_{i}$ for some $i \in\{1, \ldots, n\}$ : Since $\beta_{l}=0$ holds for all $l>k$ and $\beta_{k}=\varphi\left(\bar{\alpha}^{(n)}\right)$, this case is equivalent to $\beta_{r}<\alpha_{r}$ and $\beta_{k}<\beta_{i}$ for some $i \in\{1, \ldots, k-1\}$. Furthermore, we have $i \in\{r+1, \ldots, k-1\}$ due to $\beta_{i}=\alpha_{i}<\beta_{k}$ for $i<r$ and $\beta_{r}<\alpha_{r}<\beta_{k}$. 
3. Case $\beta_{r}<\alpha_{r}$ and $\varphi\left(\bar{\alpha}^{(n)}\right)=\beta_{i}$ holds with $\beta_{j} \neq 0$ for some $i, j \in\{1, \ldots, n\}$ with $i<j$ : Since $\beta_{l}=0$ holds for all $l>k$, we have that $i<k$ holds. With this and since $\varphi\left(\bar{\alpha}^{(n)}\right)=\beta_{k} \neq 0$ holds, we can always choose $j=k$ and get that the current case is equivalent to $\beta_{r}<\alpha_{r}$ and $\beta_{k}=\beta_{i}$ for some $i \in\{1, \ldots, k-1\}$. Furthermore, we have $i \in\{r+1, \ldots, k-1\}$ due to $\beta_{i}=\alpha_{i}<\beta_{k}$ for $i<r$ and $\beta_{r}<\alpha_{r}<\beta_{k}$.

Remark 5.20. In order to simplify the formulation and proof of Lemma 5.19, we took the lists of ordinals $\alpha_{1}, \ldots, \alpha_{n}$ and $\beta_{1}, \ldots, \beta_{n}$ to have the same length $n \geq 2$. Clearly, the lemma holds analogously for lists of ordinals with different length $\geq 2$ (just add ordinals of the form 0 to the front of the shorter list to make them the same length).

\section{The $\vartheta$-function}

See [RW93] and [Buc16] for details on the definition and properties of the function $\vartheta:$ On $\rightarrow$ On. We state only the notation $\Omega(n, x)$ that is defined for each $x \in$ On and each $n \in \mathbb{N}$ by

$$
\Omega(0, x):=\Omega \cdot x \quad \Omega(1, x):=\Omega^{x} \quad \Omega(n+1, x):=\Omega^{\Omega(n, x)}
$$

and cite the following properties in order to relate the $\vartheta$-function to the setting of Klammersymbols when we present results from the literature in Section 7. By using the Buchholz $\psi$-functions from [BS88] or the Feferman-Aczel $\theta$-functions from [Bri75], we also have the following correspondence $\vartheta \Omega^{\omega}=\psi_{0} \Omega^{\Omega^{\omega}}=\theta \Omega^{\omega} 0$. See also the last paragraph in [Rat92].

Proposition 5.21. Let $f:$ On $\rightarrow$ On, $\xi \mapsto \omega^{\xi}$.

(a) $f\left(\begin{array}{c}1 \\ \omega\end{array}\right)$ denotes the small Veblen ordinal.

(b) $f\left(\begin{array}{c}1 \\ \omega\end{array}\right)=\vartheta \Omega^{\omega}$ and $\varphi(\omega, 0)=f\left(\begin{array}{c}\omega \\ 1\end{array}\right)=\vartheta(\Omega \cdot \omega)$.

Proof. (a) follows essentially from Definition 5.4, Lemma 5.14, and Proposition 5.13. (b) is due to [Sch92], see also [Buc16] or [Ran15, Chapter 2].

\subsection{Fundamental Sequences and Ordinal Notations}

As described in the appendix of this article (see Section A) and carried out in [Ran15, Chapter $3]$ with proofs and descriptions in detail, it is possible to define a primitive recursive ordinal notation system $(\mathrm{OT}, \prec)$ for the small Veblen ordinal that is based on the finitary Veblen functions (e.g., by using codes of the form $\phi \bar{a}^{(n+1)}$ ) and whose basic properties (such as being a linear ordering) can be formalized and verified within TID. Moreover, it is possible to define a sensible semantics for ordinal notations $a \in \mathrm{OT}$ by means of a function o: OT $\rightarrow$ On such that in particular o $\left(\phi \bar{a}^{(n+1)}\right)=\varphi\left(\mathrm{o}\left(a_{1}\right), \ldots, \mathrm{o}\left(a_{n+1}\right)\right)$ holds for $\phi \bar{a}^{(n+1)} \in \mathrm{OT}$. See [Ran15, Definition 3.20] for all details.

Given this interpretation, we can define primitive recursive functions on the natural numbers $\mathbb{N}$ such as $\tilde{\varphi}, \tilde{\omega}, \tilde{\cdot}, \tilde{+}$ that act as proof-theoretic counterparts to the set-theoretic functions $\varphi$, $\omega, \cdot,+$ on the ordinals On. They simulate the ordinal arithmetic on the codes based on OT. For instance for $\varphi$ or to be more precise for $\varphi_{n+1}$ and $n \in \mathbb{N}$, we have that

- $\varphi_{n+1}: \mathrm{On}^{n+1} \rightarrow$ On is the $n+1$-ary ordinal-theoretic Veblen function. 
- $\phi: \mathbb{N} \rightarrow \mathbb{N}$ is a unary primitive recursive function whose purpose is to produce Gödel numbers $\phi(x)$ for (codes of) lists of notations $x=\left\langle a_{1}, \ldots, a_{n+1}\right\rangle$ with $a_{1}, \ldots, a_{n+1} \in$ OT. When formally defining OT, only certain codes $\phi(x)$ are allowed to be in OT. We write $\phi a_{1} \ldots a_{n+1}$ to denote $\phi\left(\left\langle a_{1}, \ldots, a_{n+1}\right\rangle\right)$. See the appendix for more details and see [Ran15] for a full definition and more explanations.

- $\tilde{\varphi}_{n+1}: \mathbb{N}^{n+1} \rightarrow \mathbb{N}$ is a primitive recursive function that simulates certain behaviors of the order-theoretic function $\varphi$ and where we have o $\left(\tilde{\varphi}\left(\bar{a}^{(n+1)}\right)\right)=\varphi\left(\mathrm{o}\left(a_{1}\right), \ldots, \mathrm{o}\left(a_{n+1}\right)\right)$ for each $a_{1}, \ldots, a_{n+1} \in \mathrm{OT}$.

Given such a setup, fundamental sequences $a[x]$ for ordinal notations $a \in \mathrm{OT}$ and natural numbers $x \in \mathbb{N}$ can be defined with the following properties (see also Definition A.1 in the appendix). The notions Suc and Lim in Proposition 5.22 below are primitive recursively defined from OT and consist of successor codes and limit codes, respectively.

More precisely, the fundamental sequences' definition as given in Definition A.1 in the appendix can be motivated from the set-theoretic perspective of Subsection 5.1. As a consequence, the following interplay of the finitary Veblen functions with the given fundamental sequences can be intuitively understood without relying on the intrinsic properties of the ordinal notation system $(\mathrm{OT}, \prec)$.

Proposition 5.22 ([Ran15, Section 3.4]).

(a) $\mathrm{TID} \vdash \forall d, x(d \in \mathrm{Suc} \rightarrow d[x] \prec d)$.

(b) $\operatorname{TID} \vdash \forall d, x(d \in \operatorname{Lim} \rightarrow(0 \prec d[x] \wedge d[x] \prec d[x+\mathbb{N} 1] \wedge d[x] \prec d))$.

(c) $\operatorname{TID} \vdash \forall d, d_{0}\left(d \in \operatorname{Lim} \wedge d_{0} \prec d \rightarrow \exists x\left(d_{0} \prec d[x]\right)\right)$.

Proposition 5.23 ([Ran15, Section 3.4]). Assume that $d_{0} \prec \tilde{\varphi}\left(\bar{a}^{(m+1)}, \overline{0}^{(k)}, b\right)$ holds for given $\bar{a}^{(m)}, b, d_{0} \in \mathrm{OT}$ and $k, m \in \mathbb{N}$. Then TID proves the following:

(a) $b \in \operatorname{Lim} \rightarrow \exists x\left(d_{0} \prec \tilde{\varphi}\left(\bar{a}^{(m+1)}, \overline{0}^{(k)}, b[x]\right)\right)$.

(b) $\left(b \notin \operatorname{Lim} \wedge a_{1}=0 \wedge \ldots \wedge a_{m+1}=0\right) \rightarrow \exists x\left(d_{0} \prec \tilde{\omega}^{b[x]} \sim(x+\mathbb{N} 1)\right)$.

(c) $\left(b=0 \wedge a_{m+1} \in \operatorname{Lim}\right) \rightarrow \exists x\left(d_{0} \prec \tilde{\varphi}\left(\bar{a}^{(m)}, a_{m+1}[x], \overline{0}^{(k+1)}\right)\right)$.

Lemma $5.24\left(\left[\operatorname{Ran} 15\right.\right.$, Section 3.2]). For $a, b, a_{1}, \ldots, a_{n+1}, c \in \mathrm{OT}$ and $n, m \in \mathbb{N}$, we have within TID:

(a) If $a \preceq c$ and $c \prec a \tilde{+} b$ hold, then $c=a \tilde{+} d$ holds for some $d \in \mathrm{OT}$ with $d \prec b$.

(b) $\tilde{\varphi}\left(\bar{x}^{(n+1)}\right) \in \mathrm{OT}$ and $x_{1}, \ldots, x_{n+1} \in \mathrm{OT}$ are equivalent for each $x_{1}, \ldots, x_{n+1} \in \mathbb{N}$.

(c) $x \tilde{+} y \in \mathrm{OT}$ and $x, y \in \mathrm{OT}$ are equivalent for each $x, y \in \mathbb{N}$.

(d) If $a_{1} \neq 0$ and $a_{m+1} \notin\{0\} \cup\left\{\phi \bar{a}^{(n+1)}: a_{1} \neq 0 \quad \& \quad a_{1}, \ldots, a_{n+1} \in \mathbb{N}\right\}$ hold, then $\tilde{\varphi}\left(\bar{a}^{(m+1)}, \overline{0}^{(k)}\right)=\phi \bar{a}^{(m+1)} \overline{0}^{(k)}$ holds.

However, having a more accessible approach to the wellordering proofs by using fundamental sequences instead of working directly with the underlying ordinal notation system has the cost that one has to verify the fundamental sequences' adequate behaviour in the background (compare [Ran15, Sections 3.4 and 11.1.2]). 


\section{Lower Bound $\vartheta \Omega^{\omega}$ for FIT and TID}

In this section, we obtain a lower bound for the proof-theoretic ordinal of the theory TID by means of wellordering proofs. Hence, together with the embedding of TID into FIT from Subsection 4.2, we automatically get a lower bound for FIT as well. We rely for the following proofs on the availability of fundamental sequences for the underlying ordinal notation system $(\mathrm{OT}, \prec)$ as described in Subsection 5.2. This allows us to shift the dependence on the specific implementation of $(\mathrm{OT}, \prec)$ to the properties of the fundamental sequences.

Alternatively, we could implement the following proofs directly within the setting of the ordinal notation system $(\mathrm{OT}, \prec)$, avoiding the introduction of fundamental sequences but for the cost of then having the ordinal notation system (OT, $\prec)$ appear more prominently in the wellordering proofs. In this case, it would be technically more sensible to work with fixed-point free variants $\bar{\varphi}_{n+1}$ of the finitary Veblen functions and base (OT, $\left.\prec\right)$ on those (see also [Sch54, $\S 3])$. We did not choose this approach for the sake of a better motivation and understanding of the main methods of the wellordering proof. For this section, we fix the following conventions:

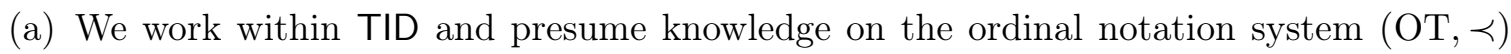
that is mentioned in the appendix and given in [Ran15, Chapter 3].

(b) The notion ordinal denotes terms that are given according to the ordinal notation system $(\mathrm{OT}, \prec)$. Small Greek letters $\alpha, \beta, \gamma, \delta, \ldots$ denote explicit terms for ordinal notations in the sense of OT and which are given externally in the meta-theory. Furthermore, we shall simplify the rigid notation that appears in the appendix and Subsection 5.2 by dropping the tilde in the expressions such as $\tilde{\varphi}, \tilde{\omega}, \tilde{+}$ and writing instead $\varphi, \omega,+$, respectively.

(c) $P$, Acc, Prog, and TI shall denote $P_{\prec}, \mathrm{Acc}_{\prec}, \operatorname{Prog}_{\prec}$, and $\mathrm{TI}_{\prec}$, respectively.

\section{Proposition 6.1.}

(a) $\mathrm{TID} \vdash \forall x(x \notin \mathrm{OT} \rightarrow P x)$.

(b) $\operatorname{TID} \vdash \forall x(P x \rightarrow \operatorname{TI}(\mathcal{A}, x))$ for all $\mathcal{A} \in \mathcal{L}_{\mathrm{PA}}$.

(c) $\operatorname{TID} \vdash \operatorname{TI}(\mathcal{A}, \alpha)$ holds for each $\alpha \prec \omega$ and $\mathcal{A} \in \mathcal{L}_{\mathrm{TID}}$.

Proof (Sketch). (a) holds immediately by $(\mathrm{Cl})$, using that $a \notin \mathrm{OT}$ implies $b \nprec a$ for all $b$. For (b), assume $P a, \operatorname{Prog}(\mathcal{A})$, and $b \prec a$. We get $P b$ by (FP) from Remark 4.16, and since $\mathcal{A} \in \operatorname{Pos}_{1}$ holds, we then get $\mathcal{A}(b)$ by (TID). For $(\mathrm{c})$, note that we can show $\operatorname{TI}\left(\mathcal{A}, \mathfrak{n}_{k}\right)$ for all $k \in \mathbb{N}$ by (meta-)induction on $k$, while we set $\mathfrak{n}_{0}:=0$ and $\mathfrak{n}_{m+1}:=\mathfrak{n}_{m} \tilde{+} \tilde{1}$ for each $m \in \mathbb{N}$.

Remark 6.2. Dropping the restriction on the induction formula used in (Ind) yields TID $\vdash$ $\operatorname{TI}(\mathcal{A}, \alpha)$ for each $\alpha \prec \varepsilon_{0}$ and $\mathcal{A} \in \mathcal{L}_{\mathrm{TID}}$. This is since TID would extend PA in this case with complete induction for the full language $\mathcal{L}_{\text {TID }}$, and we could then follow the usual wellordering proofs for PA, adapting them to the representation of ordinals below $\varepsilon_{0}$ as given here.

Remark 6.3. Due to Proposition 6.1.(a), we can assume from now on without loss of generality that $a \in$ OT holds whenever we try to show $P a$ for some $a$ within TID. In particular, if we aim to prove $P(a+b)$ or $P \varphi\left(\bar{a}^{(n)}\right)$ for some $a, b, a_{1}, \ldots, a_{n}$ (with $n \geq 1$ ), we tacitly assume that $a+b \in \mathrm{OT}$ and $\varphi\left(\bar{a}^{(n)}\right) \in \mathrm{OT}$ hold, respectively. Due to Lemma 5.24, we also get $a, b, a_{1}, \ldots, a_{n} \in$ OT. Since $x \prec y$ implies $x, y \in$ OT for all $x, y$ by the definition of $\prec$, statements such as $c \prec a+b$ or $c \prec \varphi\left(\bar{a}^{(n)}\right)$ imply $a+b \in$ OT and $\varphi\left(\bar{a}^{(n)}\right) \in$ OT, respectively. 
Lemma 6.4. TID $\vdash \forall x, y(P x \wedge P y \rightarrow P(x+y))$.

Proof. Assume $a_{1}, a_{2}$ with $P a_{1}$ and $P a_{2}$, so we have to show $P\left(a_{1}+a_{2}\right)$. By showing $\operatorname{Prog}(\mathcal{B})$ for $\mathcal{B}:=\Lambda b . P\left(a_{1}+b\right)$, we can use (TID) together with $P a_{2}$ to get the claim. Now, $\operatorname{Prog}(\mathcal{B})$ is $\forall z(\operatorname{Acc}(\mathcal{B}, z) \rightarrow \mathcal{B}(z))$, so assume $c$ and $\operatorname{Acc}(\mathcal{B}, c)$, i.e., $\forall z \prec c\left(P\left(a_{1}+z\right)\right)$. Due to $(\mathrm{Cl})$, it suffices to show $\left(\forall z \prec a_{1}+c\right)(P z)$. Let now $d \prec a_{1}+c$. We have either $d \prec a_{1}$ and can use (FP) on assumption $P a_{1}$ to get $P d$, or otherwise, we have $a_{1} \preceq d \prec a_{1}+c$ by Lemma 5.24. In the latter case, we have $d=a_{1}+c_{0}$ for some $c_{0} \prec c$, so our assumptions yield the claim.

\subsection{The Simple Case for the Binary Veblen Function}

We treat the case for the binary Veblen function separately in order to give a more transparent proof that avoids the technicalities that appear in the treatment of the general case in Subsection 6.2 (such as the use of auxiliary class terms of the form $\operatorname{Part}_{n}^{k}$ for $1 \leq k \leq n$ ).

Lemma 6.5. TID $\vdash \forall x, y(P x \wedge P y \rightarrow P \varphi(x, y))$.

Proof. Note that $P 0$ and hence $P 1$ hold due to $(\mathrm{Cl})$. Now, we assume $a_{1}, a_{2}$ with $P a_{1}, P a_{2}$. We use the class term $\mathcal{B}:=\Lambda a \cdot \forall y(P y \rightarrow P \varphi(a, y))$ with $\mathcal{B} \in \operatorname{Pos}_{1}$ and show $\operatorname{Prog}(\mathcal{B})$. Then we can use (TID) with $P a_{1}$ and $P a_{2}$. Now, in order to bring the proof of this lemma closer to the proof of Theorem 6.12 that deals with the general case of a finitary Veblen function, we note that $\operatorname{Prog}(\mathcal{B})$ is $\forall z(\forall x \prec z(\mathcal{B}(x)) \rightarrow \mathcal{B}(z))$ Now, using the class term $\mathcal{A}_{2}^{1}:=\Lambda a . \forall y(P y \rightarrow \forall x \prec a(P \varphi(x, y)))$ and that $\mathcal{A}_{2}^{1}(a)$ is logically equivalent to $\forall x \prec a(\mathcal{B}(x))$, we get that $\operatorname{Prog}(\mathcal{B})$ is logically equivalent to $\forall z\left(\mathcal{A}_{2}^{1}(z) \rightarrow \mathcal{B}(z)\right)$ So, in order ot show the latter, assume $a$ with

$$
\mathcal{A}_{2}^{1}(a)
$$

and show $\mathcal{B}(a)$, while for proving $\mathcal{B}(a)$, assume $b$ with $P b$ and show $P \varphi(a, b)$. Once more, we can use (TID), namely with $\mathcal{A}_{2}^{2}:=\Lambda d . P \varphi(a, d)$ on assumption $P b$ since $\mathcal{A}_{2}^{2} \in \mathrm{Pos}_{1}$ holds, while we have to show $\operatorname{Prog}\left(\mathcal{A}_{2}^{2}\right) .{ }^{11}$ Now, for proving $\operatorname{Prog}\left(\mathcal{A}_{2}^{2}\right)$, we assume $d$ and $z$ with

$$
\forall z_{0} \prec d\left(\mathcal{A}_{2}^{2}\left(z_{0}\right)\right) \quad\left(\text { i.e., } \forall z_{0} \prec d\left(P \varphi\left(a, z_{0}\right)\right)\right)
$$

and $z \prec \varphi(a, d)$, having to show $P z$. This yields $P \varphi(a, d)$ by $(\mathrm{Cl})$ because $z$ is arbitrary. We consider now the following case distinction.

1. If $d \in$ Lim: We get that $z \prec \varphi(a, d[x])$ holds for some $x$ by Proposition 5.23. Since we have $d[x] \prec d$ by Proposition 5.22 , we get $P \varphi(a, d[x])$ by (8), implying $P z$ by (FP).

2. If $d \notin \mathrm{Lim}$ :

2.1. If $a=0$ : Since $d \notin$ Lim holds, we get $z \prec t(x)$ for some $x$ by Proposition 5.23, where we let $t(x):=\omega^{d[x]} \cdot(x+\mathbb{N} 1)$ We show $\forall x(P(t(x)))$ by induction on $x$ and note that (Ind) is applicable here because of $\Lambda x . P(t(x)) \in \operatorname{Pos}_{1}$. For $x=0$, we can argue as for the case $d \in \operatorname{Lim}$ and get $P\left(\omega^{d[0]}\right)$. For $x=x_{0}+\mathbb{N} 1$, the claim follows from $P(t(0))$, the induction hypothesis, and Lemma 6.4, noting that $d[0]=d\left[x_{0}\right]$ holds by definition and because of $d \notin \mathrm{Lim}$.

\footnotetext{
${ }^{11}$ Noting our current assumption (7) and our current goal, we remark that we actually show$$
\mathcal{A}_{2}^{1}(a) \rightarrow \operatorname{Prog}\left(\mathcal{A}_{2}^{2}\right)
$$

which is a special case of Theorem 6.10 , and also note that $\mathcal{A}_{2}^{1} \in \operatorname{Pos}_{1}$ holds with $\mathcal{A}_{2}^{1} \notin \operatorname{Pos}_{0}$, while we have $\mathcal{A}_{2}^{2} \in \mathrm{Pos}_{0}$.
} 
2.2. If $a \in \operatorname{Lim}$ and $d=0$ : We have by Proposition 5.23 that $z \prec \varphi(a[x], 0)$ holds for some $x$. Since we have $a[x] \prec a$ by Proposition 5.22 , we get $P \varphi(a[x], 0)$ with $(7)$.

2.3. Otherwise, i.e., either $d=0$ with $a \in$ Suc or $d \in$ Suc with $a \neq 0$ : Letting $t:=\varphi(a, d)$, we have by Proposition 5.22 some $x$ such that $z \prec t[x]$ holds. Proving $\forall x(P(t[x]))$ by induction on $x$ suffices now. Note again that (Ind) is applicable due to $\Lambda x . P(t[x]) \in \operatorname{Pos}_{1}$, and also note for the following computations of $t[x]$ according to Definition A.1 in the appendix that we have $\varphi(a, d)=\phi a d$ by Lemma 5.24 , bearing in mind that $\phi$ is from the ordinal notation system $(\mathrm{OT}, \prec)$.

2.3.1. If $x=0$ : If $d=0$ holds, then we have $t[0]=1$ and are done since we have $P 1$. If

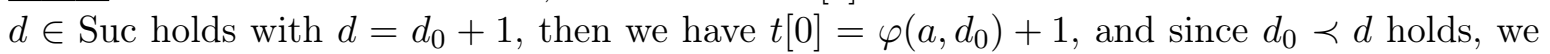
get $P(t[0])$ from (8) and Lemma 6.4 by using $P 1$.

2.3.2. If $x=x_{0}+_{\mathbb{N}} 1$ : We have $t\left[x_{0}+_{\mathbb{N}} 1\right]=\varphi\left(a\left[x_{0}\right], t\left[x_{0}\right]\right)$, so the claim follows with $a\left[x_{0}\right] \prec a$ from Proposition 5.22, the induction hypothesis $P\left(t\left[x_{0}\right]\right)$, and (7).

Corollary 6.6. TID $\vdash P \varphi(\alpha, 0)$ holds for each $\alpha \prec \omega$.

Proof. The claim is a direct consequence of Lemma 6.5. Note that $P \alpha$ for $\alpha \prec \omega$ follows from Proposition 6.1.(c): TI $(\Lambda a . P a, \alpha)$ and (Cl) imply $\forall x \prec \alpha(P x)$, hence $P \alpha$ by (FP).

Remark 6.7. We proved Lemma 6.5 by applying (TID) to a class term $\mathcal{B}$ in $\operatorname{Pos}_{1}$ that is not in $\operatorname{Pos}_{0}$. Though, in order to $\operatorname{show} \operatorname{Prog}(\mathcal{B})$ in the proof of Lemma 6.5, we can work with a (weaker) subtheory TID $_{0}$ of TID that is obtained from TID by restricting (the instances of) the axiom schemes (TID) and (Ind) to class terms that are in $\operatorname{Pos}_{0}$ (rather than $\operatorname{Pos}_{1}$ ), see [Ran15, Chapter 8] for details. This theory $\operatorname{TID}_{0}$ is the restriction of the theory $\mathrm{ID}_{1}^{*} \uparrow$ to accessible part positive operator forms, i.e., to the language $\mathcal{L}_{\text {TID }}$, while $\left.I D_{1}^{*}\right\rceil$ is a subtheory of $I D_{1}$ for positive induction and with the same restriction for complete induction. The proof-theoretic ordinal of $\mid \mathrm{ID}_{1}^{*} \uparrow$ is $\varphi(\omega, 0)$, see for instance [Pro06]. Note furthermore that the proof of Theorem 6.10 below does not invoke (TID), so this result holds also for the restriction TID $_{0}$.

\subsection{The General Case for the Finitary Veblen Functions}

Definition 6.8. For $k, n \in \mathbb{N}$ with $1 \leq k<n$, we define

$$
\begin{aligned}
\operatorname{Part}_{n}^{k} & :=\Lambda \bar{a}^{(k)} \cdot \forall y\left(P y \rightarrow \forall x \prec a_{k}\left(P \varphi\left(\bar{a}^{(k-1)}, x, y, \overline{0}^{(n-k-1)}\right)\right)\right) \\
\operatorname{Hyp}_{n}^{k}: & =\Lambda \bar{a}^{(k)} \cdot \operatorname{Part}_{n}^{1}\left(a_{1}\right) \wedge \ldots \wedge \operatorname{Part}_{n}^{k}\left(\bar{a}^{(k)}\right) \\
\operatorname{Hyp}_{n}^{0} & :=(0=0)
\end{aligned}
$$

Lemma 6.9. For $k, n \in \mathbb{N}$ and variables $a_{1}, \ldots, a_{n-1}$, the following holds:

(a) $\left(\Lambda a . P\left(\varphi\left(\bar{a}^{(n-1)}, a\right)\right)\right) \in \operatorname{Pos}_{0}$ for $1 \leq n$.

(b) $\left(\Lambda a \cdot \operatorname{Part}_{n}^{k}\left(\bar{a}^{(k-1)}, a\right)\right) \in \operatorname{Pos}_{1}$ for $1 \leq k<n$.

Proof. (a) is obvious. For (b), note in the definition of $\operatorname{Part}_{n}^{k}\left(\bar{a}^{(k-1)}, a\right)$ that $P y$ and $\forall x(x \prec$ $\left.a \rightarrow P \varphi\left(\bar{a}^{(k-1)}, x, y, \overline{0}^{(n-k-1)}\right)\right)$ are in $P_{0 s_{0}}$. Furthermore, $P y$ does not contain $a$ as a free variable, so we get indeed that $\forall y\left(P y \rightarrow \forall x \prec a\left(P \varphi\left(\bar{a}^{(k-1)}, x, y, \overline{0}^{(n-k-1)}\right)\right)\right)$ is in $\operatorname{Pos}_{1}(a)$.

Theorem 6.10. TID $\vdash \forall \bar{a}^{(n-1)}\left(\operatorname{Hyp}_{n}^{n-1}\left(\bar{a}^{(n-1)}\right) \rightarrow \operatorname{Prog}\left(\Lambda a \cdot P \varphi\left(\bar{a}^{(n-1)}, a\right)\right)\right)$ holds for $n \geq 1$. 
Proof. Assume $n \geq 1$ and $\bar{a}^{(n-1)}$ with $\operatorname{Hyp}_{n}^{n-1}\left(\bar{a}^{(n-1)}\right)$. For showing $\operatorname{Prog}\left(\Lambda a \cdot P \varphi\left(\bar{a}^{(n-1)}, a\right)\right)$, assume $a$ and $d$ such that $d \prec \varphi\left(\bar{a}^{(n-1)}, a\right)$ and

$$
\forall x \prec a\left(P \varphi\left(\bar{a}^{(n-1)}, x\right)\right)
$$

hold and show $P d$. This would yield $P \varphi\left(\bar{a}^{(n-1)}, a\right)$ by $(\mathrm{Cl})$ because $d$ is arbitrary.

1. If $n=1$ or $a_{1}=\ldots=a_{n-1}=0$ hold: As in Lemma 6.5 due to $\varphi\left(\bar{a}^{(n-1)}, a\right)=\varphi(a)=\omega^{a}$.

2. Otherwise: We can assume now that some $1 \leq l \leq n-1$ exists with $a_{l} \neq 0$ and $a_{l+1}=$ $\ldots=a_{n-1}=0$, i.e., that we have $\varphi\left(\bar{a}^{(n-1)}, a\right)=\varphi\left(\bar{a}^{(l)}, \overline{0}^{(n-l-1)}, a\right)$ with $a_{l} \neq 0$. Furthermore, our assumption $\operatorname{Hyp}_{n}^{n-1}\left(\bar{a}^{(n-1)}\right)$ yields

$$
\operatorname{Part}_{n}^{1}\left(a_{1}\right) \wedge \ldots \wedge \operatorname{Part}_{n}^{n-1}\left(\bar{a}^{(n-1)}\right)
$$

Consider now the following case distinction and note that $P 0$ and hence $P 1$ hold due to $(\mathrm{Cl})$. 2.1. If $a \in \mathrm{Lim}$ : We get that $d \prec \varphi\left(\bar{a}^{(n-1)}, a[x]\right)$ holds for some $x$ by Proposition 5.23 and assumption $d \prec \varphi\left(\bar{a}^{(n-1)}, a\right)$. Since we have $a[x] \prec a$ by Proposition 5.22, we get $P \varphi\left(\bar{a}^{(n-1)}, a[x]\right)$ by (9) which implies $P d$ by (FP).

2.2. If $a \notin \mathrm{Lim}$ :

2.2.1. If $a_{l} \in \operatorname{Lim}$ and $a=0$ : By Proposition 5.23, we have $z \prec \varphi\left(\bar{a}^{(l-1)}, a_{l}[x], \overline{0}^{(n-l)}\right)$ for some $x$. We get $P \varphi\left(\bar{a}^{(l-1)}, a_{l}[x], \overline{0}^{(n-l)}\right)$ with $\operatorname{Part}_{n}^{l}\left(\bar{a}^{(l)}\right)$ from (10) because we have $a_{l}[x] \prec a_{l}$ by Proposition 5.22 .

2.2.2. Otherwise, i.e., either $a=0$ with $a_{l} \in$ Suc or $a \in$ Suc with $a_{l} \neq 0$ : In this situation, Lemma 5.24 implies $\varphi\left(\bar{a}^{(l)}, \overline{0}^{(n-l)}\right)=\phi a_{p} \ldots a_{l} \overline{0}^{(n-l-1)} a$ for some $1 \leq p \leq l$ where $a_{1}, \ldots, a_{p}=$ 0 holds. For the computation of $t[x]$, see Definition A.1 in the appendix and bear in mind that $\phi$ is from the ordinal notation system $(\mathrm{OT}, \prec)$. In order to simplify notation and without loss of generality, we shall assume $p=1$, noting that the following argument works for the general case as well. Letting $t:=\phi \bar{a}^{(l)} \overline{0}^{(n-l-1)} a$ we have by Proposition 5.22 some $x$ such that $z \prec t[x]$ holds. Proving $\forall x(P(t[x]))$ by induction on $x$ suffices now. (Ind) is applicable because $\Lambda x . P(t[x]) \in \operatorname{Pos}_{1}$ holds.

2.2.2.1. If $x=0$ : For $a=0$, we have $t[0]=1$ and are done since we have $P 1$. If $a \in$ Suc holds with $a=a_{0}+1$, then we have $t[0]=\varphi\left(\bar{a}^{(n-1)}, a_{0}\right)+1$ due to the form of $t$ and the definition of $t[0]$. Since $a_{0} \prec a$ holds, we get $P(t[0])$ from (9) and Lemma 6.4 by using $P 1$.

2.2.2.2. If $x=x_{0}+_{\mathbb{N}} 1$ : We get $t\left[x_{0}+\mathbb{N} 1\right]=\varphi\left(\bar{a}^{(l-1)}, a_{l}\left[x_{0}\right], t\left[x_{0}\right], \overline{0}^{(n-l)}\right)$, so the claim follows with $a_{l}\left[x_{0}\right] \prec a_{l}$ from Proposition 5.22, the induction hypothesis $P\left(t\left[x_{0}\right]\right)$, and $\operatorname{Part}_{n}^{l}\left(\bar{a}^{(l)}\right)$ from (10).

Corollary 6.11. TID $\vdash \forall \bar{a}^{(n)}\left(\operatorname{Hyp}_{n}^{n-1}\left(\bar{a}^{(n-1)}\right) \wedge P a_{n} \rightarrow P \varphi\left(\bar{a}^{(n)}\right)\right)$ for $n \in \mathbb{N}$ with $n \geq 1$.

Proof. Immediate from Theorem 6.10 by using (TID) and Lemma 6.9.(a).

Theorem 6.12. TID $\vdash \forall \bar{a}^{(k-1)}\left(\operatorname{Hyp}_{n}^{k-1}\left(\bar{a}^{(k-1)}\right) \rightarrow \operatorname{Prog}\left(\Lambda a \cdot \operatorname{Part}_{n}^{k}\left(\bar{a}^{(k-1)}, a\right)\right)\right)$ for $1 \leq k<n$.

Proof. We fix $n \geq 1$ and argue by induction on $n-k$ for $1 \leq k<n$. Let $\bar{a}^{(k-1)}$ be given with $\operatorname{Hyp}_{n}^{k-1}\left(\bar{a}^{(k-1)}\right)$, while note that this just gives us the formula $0=0$ in case of $k=1$. Assume now $a, a_{k}, a_{k+1}$ with

$$
\forall x \prec a\left(\operatorname{Part}_{n}^{k}\left(\bar{a}^{(k-1)}, x\right)\right) \wedge P a_{k+1} \wedge a_{k} \prec a
$$


in order to show $\operatorname{Prog}\left(\Lambda a \cdot \operatorname{Part}_{n}^{k}\left(\bar{a}^{(k-1)}, a\right)\right)$. In case we have $k \neq n-1$, further let $a_{j}:=0$ for each $k+1<j \leq n$. We have to show $P\left(\varphi\left(\bar{a}^{(k+1)}, \overline{0}^{(n-k-1)}\right)\right)$, i.e.,

$$
P\left(\varphi\left(\bar{a}^{(n)}\right)\right)
$$

For this, we show by a side induction on $i$ that $1 \leq i<n$ implies $\operatorname{Hyp}_{n}^{i}\left(\bar{a}^{(i)}\right)$. Then for $i:=n-1$, we get $(*)$ by Corollary 6.11 since we have $P a_{n}$ by (11) or (if $k=n-1$ ) by $P 0$.

For the remaining claim, note that $\operatorname{Part}_{n}^{k}\left(\bar{a}^{(k-1)}, a_{k}\right)$ holds by (11) and so $\operatorname{Hyp}_{n}^{k}\left(\bar{a}^{(k)}\right)$ by definition, using assumption $\operatorname{Hyp}_{n}^{k-1}\left(\bar{a}^{(k-1)}\right)$. We use this argument in case of $1 \leq i \leq k$. If we have $k<i<n$, then we can use the side induction hypothesis and get $\operatorname{Hyp}_{n}^{i-1}\left(\bar{a}^{(i-1)}\right)$. So, the main induction hypothesis yields $\operatorname{Prog}\left(\Lambda a \cdot \operatorname{Part}_{n}^{i}\left(\bar{a}^{(i-1)}, a\right)\right)$ and hence we get $\forall a(P a \rightarrow$ $\operatorname{Part}_{n}^{i}\left(\bar{a}^{(i-1)}, a\right)$ ) by (TID), while noting Lemma 6.9.(b). Now, $\operatorname{Part}_{n}^{i}\left(\bar{a}^{(i)}\right)$ follows from $P a_{k+1}$ in (11) in case of $i=k+1$ and otherwise from $P 0$. Hence, we get $\operatorname{Hyp}_{n}^{i}\left(\bar{a}^{(i)}\right)$ by $\operatorname{Hyp}_{n}^{i-1}\left(\bar{a}^{(i-1)}\right)$.

Corollary 6.13. TID $\vdash \forall \bar{a}^{(k)}\left(\operatorname{Hyp}_{n}^{k-1}\left(\bar{a}^{(k-1)}\right) \wedge P a_{k} \rightarrow \operatorname{Hyp}_{n}^{k}\left(\bar{a}^{(k)}\right)\right)$ holds for all $k, n \in \mathbb{N}$ with $1 \leq k<n$.

Proof. From $\operatorname{Hyp}_{n}^{k-1}\left(\bar{a}^{(k-1)}\right) \wedge P a_{k}$, we get $\operatorname{Part}_{n}^{k}\left(\bar{a}^{(k)}\right)$ by Theorem 6.12 and (TID), while noting Lemma 6.9.(b). Hence, we get $\operatorname{Hyp}_{n}^{k}\left(\bar{a}^{(k)}\right)$.

Theorem 6.14. TID $\vdash \forall \bar{a}^{(n)}\left(\bigwedge_{i=1}^{n} P a_{i} \rightarrow P \varphi\left(\bar{a}^{(n)}\right)\right)$ holds for each $n \geq 1$.

Proof. Let $n \geq 1$ and assume $\bar{a}^{(n)}$ with $\bigwedge_{i=1}^{n} P a_{i}$. We now show by induction on $k \in \mathbb{N}$ that $0 \leq k<n$ implies $\operatorname{Hyp}_{n}^{k}\left(\bar{a}^{(k)}\right) \wedge \bigwedge_{i=k+1}^{n} P a_{i}$. Then the claim $P \varphi\left(\bar{a}^{(n)}\right)$ follows from this and Corollary 6.11 with $k:=n-1$. So assume $0 \leq k<n$. If $k=0$, we trivially get $\operatorname{Hyp}_{n}^{0}\left(\bar{a}^{(0)}\right) \wedge \bigwedge_{i=1}^{n} P a_{i}$ due to the definition of $\operatorname{Hyp}_{n}^{0}$ and our assumption $\bigwedge_{i=1}^{n} P a_{i}$. If $0<k \leq n$, the induction hypothesis yields $\operatorname{Hyp}_{n}^{k-1}\left(\bar{a}^{(k-1)}\right) \wedge \bigwedge_{i=k}^{n} P a_{i}$ and hence the claim $\operatorname{Hyp}_{n}^{k}\left(\bar{a}^{(k)}\right) \wedge \bigwedge_{i=k+1}^{n} P a_{i}$ due to Corollary 6.13 .

Corollary 6.15 (Lower bound of TID). $\operatorname{TID} \vdash \operatorname{TI}(\mathcal{A}, \alpha)$ holds for each $\mathcal{A} \in \mathcal{L}_{\mathrm{PA}}$ and $\alpha \in \mathrm{OT}$.

Proof. By induction on the build-up of $\alpha \in \mathrm{OT}$ as described in the appendix. We can use Lemma 6.4 and Theorem 6.14 together with Proposition 6.1.(b).

\section{Upper Bound $\vartheta \Omega^{\omega}$ for FIT and TID}

For determining the upper bound of FIT, we apply one result from [JS99] that relates over ACA $_{0}$ the scheme $\left(\Pi_{3}^{1}\right.$-RFN) of $\omega$-model reflection for $\Pi_{3}^{1}$ formulas to the scheme $\left(\Pi_{2}^{1}-\mathrm{BI}\right)$ of bar induction for $\Pi_{2}^{1}$ formulas, and one result of [RW93] that determines the proof-theoretic ordinal of $\Pi_{2}^{1}-\mathrm{BI}_{0}$ to be the small Veblen ordinal $\vartheta \Omega^{\omega}$. Then an embedding of FIT into the second order theory $\Pi_{3}^{1}$-RFN $N_{0}$ of $\omega$-model reflection for $\Pi_{3}^{1}$ formulas suffices to get the desired upper bound result for FIT. Moreover and due to Section 4.2, this also provides an upper bound for TID. In particular, we shall exploit the $\Pi_{1}^{1}$ definability of a least fixed-point. A similar approach has been taken in [AR10] and [Pro06] for the treatment of the theories $\Pi_{2}^{1}-\mathrm{RFN}_{0}$ and $\mathrm{ID}_{1}^{*}$ (a subsystem of $\mathrm{ID}_{1}$ that allows only positive induction for the predicates $P_{\mathfrak{A}}$ that are assigned to each positive operator form $\mathfrak{A}$ ). Below, we shall provide an upper bound for FIT by embedding it directly into $\Pi_{3}^{1}-\mathrm{RFN}_{0}$. We remark that if we were to investigate only the subtheory TID of $I_{1}$, we could have embedded it directly into $\Pi_{3}^{1}-\mathrm{RFN}_{0}$ (rather than 
taking the detour via FIT as Figure 1 from Section 1 suggests). Furthermore, we recall that D. Probst's modular ordinal analysis from [Pro17] determines the proof-theoretic ordinal of $\Pi_{3}^{1}-\mathrm{RFN}_{0}$ to be the small Veblen ordinal by metapredicative methods.

\subsection{Subsystems of Second Order Arithmetic}

We shall introduce here subsystems of second order arithmetic, and we formulate them in the language $\mathcal{L}_{\mathrm{PA}}^{2}$ that we defined in Section 4 . In particular, recall that $\mathcal{L}_{\mathrm{PA}}^{2}$ formulas allow for quantification over set variables $X$. The following definitions are taken to some extent from [JS99] and [Sim09], respectively, and we refer to these sources for more details on subsystems of second order arithmetic and in particular to the underlying two-sorted logic.

Definition 7.1. We use the following standard abbreviations

$$
\begin{aligned}
(X)_{t} & :=\Lambda a .\langle t, a\rangle \in X & & \\
(Q Y \dot{\in} X) A & :=(Q y) A\left((X)_{y} / Y\right) & & \text { (where } Q \in\{\forall, \exists\}) \\
Y \dot{\in} X & :=(\exists Z \dot{\in} X)(Z=Y) & & \text { (i.e., } \left.Y \dot{\in} X \text { is } \exists z\left((X)_{z}=Y\right)\right)
\end{aligned}
$$

By $A^{X}$ or also $X \models A$, we denote the (canonical) relativization of a formula $A$ to a set variable $X$, which is defined inductively as follows: If $A$ is an atomic formula, then $A^{X}:=A$. If $A$ is $\neg A_{0}$, then $A^{X}:=\neg\left(A_{0}^{X}\right)$. If $A$ is $A_{0} \circ A_{1}$ with $\circ \in\{\vee, \wedge, \rightarrow\}$, then $A^{X}:=A_{0}^{X} \circ A_{1}^{X}$. If $A$ is $(Q x) A_{0}$ with $Q \in\{\exists, \forall\}$, then $A^{X}:=(Q x) A_{0}^{X}$. If $A$ is $(Q X) A_{0}$ with $Q \in\{\exists, \forall\}$, then $A^{X}:=(Q Y \dot{\in} X) A_{0}^{X}$.

As usual, we assume tacitly a renaming of bound variables in order to avoid a clash of variables. Note that set variables occur at most free in $A^{X}$, i.e., $A^{X}$ is arithmetical.

Definition 7.2. $A \in \Pi_{0}^{1}$ and $A \in \Sigma_{0}^{1}$ denotes that $A$ is arithmetical, i.e., $A$ is a $\Pi_{0}^{1}$ (or also $\Sigma_{0}^{1}$ ) formula, i.e., a $\mathcal{L}_{\mathrm{PA}}^{2}$ formula without quantifications over set variables. $A \in \Pi_{n}^{1}$ denotes that $A$ is a $\Pi_{n+1}^{1}$ formulas, i.e., a formula which is of the form $\forall X_{1} \exists X_{2} \ldots\left(Q_{n+1} X_{n+1}\right) B$ for some $B \in \Pi_{0}^{1}$, and where $Q_{n+1}$ is $\exists$ for even $n$ and $Q_{n+1}$ is $\forall$ otherwise. $A \in \Sigma_{n}^{1}$ denotes that $A$ is a $\Sigma_{n+1}^{1}$ formulas, i.e., of the form $\exists X B$ with $B \in \Pi_{n}^{1}$.

Definition 7.3. $A C A_{0}$ is the two-sorted theory based on $\mathcal{L}_{\mathrm{PA}}^{2}$ and the axioms of PA without complete induction, where the equality axioms (for the first sort) hold for the language $\mathcal{L}_{\mathrm{PA}}^{2}$. Moreover, $\mathrm{ACA}_{0}$ consists of set induction, i.e., $\forall X((0 \in X \wedge \forall x(x \in X \rightarrow \mathbf{S} x \in X)) \rightarrow \forall x(x \in$ $X)$ ), and of arithmetical comprehension (ACA), i.e., $\exists X \forall x(x \in X \leftrightarrow A)$ for each $A \in \Pi_{0}^{1}$ that does not contain $X$.

Proposition 7.4. $\mathrm{ACA}_{0}$ is finitely axiomatizable by a $\Pi_{2}^{1}$-sentence $\mathrm{F}_{\mathrm{ACA}}$.

Proof. See for instance [Sim09, Lemma VIII.1.5].

Definition 7.5. We define the following theories and principles:

- $\Sigma_{1}^{1}-\mathrm{AC}_{0}$ is $\mathrm{ACA}_{0}$ extended by the principle of $\Sigma_{1}^{1}$ axiom of choice $\left(\Sigma_{1}^{1}-\mathrm{AC}\right)$, i.e., $\forall x \exists X A \rightarrow$ $\exists Y \forall x\left(A\left((Y)_{x} / X\right)\right)$ for each $A \in \Sigma_{1}^{1}$.

- $\Pi_{n}^{1}-\mathrm{RFN}_{0}$ is $\mathrm{ACA}_{0}$ extended by the principle of $\Pi_{n}^{1} \omega$-model reflection for $n \in \mathbb{N}$, i.e, for each $A \in \Pi_{n}^{1}$ with at most $U_{1}, \ldots, U_{k}$ occurring as free set variables in $A$ (and where $\mathrm{F}_{\mathrm{ACA}}$ is taken from Proposition 7.4), the principle is the formula $\forall U_{1}, \ldots, U_{k}(A \rightarrow$ $\left.\exists X\left(A^{X} \wedge \mathrm{F}_{\mathrm{ACA}}^{X} \wedge U_{1} \dot{\epsilon} X \wedge \ldots \wedge U_{k} \dot{\in} X\right)\right)$.

For the principles $\left(\Pi_{n}^{1}-\mathrm{BI}_{0}\right)$ of $\Pi_{n}^{1}$ bar induction and $\left(\Sigma_{1}^{1}-\mathrm{DC}\right)$ of $\Sigma_{1}^{1}$ axiom of dependent choice and their corresponding theories $\Pi_{n}^{1}-\mathrm{BI}_{0}$ and $\Sigma_{1}^{1}-\mathrm{DC} \mathrm{C}_{0}$, see the references made in Subsection 7.2. 


\subsection{Upper Bound Results from the Literature}

Theorem 7.6. $\left|\Pi_{n+2}^{1}-\mathrm{BI}_{0}\right|=\vartheta \Omega(n+1, \omega)$ holds for all $n \in \mathbb{N}$.

Proof. See [RW93, Theorem 10.2.].

Theorem 7.7. $\left(\Pi_{n+1}^{1}-\mathrm{BI}\right)$ and $\left(\Pi_{n+2}^{1}-\mathrm{RFN}\right)$ are equivalent over $\mathrm{ACA}_{0}$ for all $n \in \mathbb{N}$.

Proof. See [JS99, Main Theorem].

Theorem 7.8. Over $\mathrm{ACA}_{0}$, we have

(a) $\left(\Pi_{n}^{1}\right.$-RFN) implies $\left(\Pi_{k}^{1}-\mathrm{RFN}\right)$ for $k \leq n$.

(b) $\left(\Pi_{2}^{1}-\mathrm{RFN}\right)$ is equivalent to $\left(\Sigma_{1}^{1}-\mathrm{DC}\right)$.

(c) $\left(\Sigma_{1}^{1}\right.$-DC) implies $\left(\Sigma_{1}^{1}-\mathrm{AC}\right)$.

Proof. See [Sim09, Theorem VIII.5.12, Lemma VII.6.6].

Corollary 7.9. $\left(\Pi_{n+2}^{1}\right.$-RFN) implies $\left(\Sigma_{1}^{1}\right.$-AC) over $\mathrm{ACA}_{0}$ for all $n \in \mathbb{N}$.

Proof. By Theorem 7.8.

Theorem 7.10. $\left|\Sigma_{1}^{1}-\mathrm{DC}_{0}\right|=\varphi(\omega, 0)$.

Proof. See the last paragraph in [Can86].

Corollary 7.11. $\left|\Pi_{n+2}^{1}-\operatorname{RFN}_{0}\right|=\vartheta \Omega(n, \omega)$ holds for all $n \in \mathbb{N}$.

Proof. For $n \geq 1$, this is immediate from Theorem 7.6 and Theorem 7.7. For $n=0$, use Theorems 7.10 and 7.8.(b) and that $\vartheta \Omega(0, \omega)=\vartheta(\Omega \cdot \omega)=\varphi(\omega, 0)$ holds by Proposition 5.21.(b).

\subsection{Some Syntactical Properties of $\mathcal{L}_{\mathrm{PA}}^{2}$ Formulas}

Definition 7.12. Let $\mathrm{T}$ be some theory of $\mathcal{L}_{\mathrm{PA}}^{2}$ as introduced in Subsection 7.1. $A \in \Pi_{n}^{1}(\mathrm{~T})$ denotes that $A$ is an $\Pi_{n}^{1}$ formula over $\mathrm{T}$, i.e., $A \in \mathcal{L}_{\mathrm{PA}}^{2}$ such that $A$ is provably equivalent over T to some formula $A^{\prime} \in \Pi_{n}^{1}$.

Remark 7.13. In case that $A \in \Pi_{n}^{1}(\mathrm{~T})$ is given for some theory $\mathrm{T}$ of $\mathcal{L}_{\mathrm{PA}}^{2}$ and we consider some $A^{\prime} \in \Pi_{n}^{1}$ that is provably equivalent over $\mathrm{T}$ to $A$, then we can assume that $A$ and $A^{\prime}$ have the same free variables, and we shall tacitly do so from now on. Moreover, if $\mathrm{T}_{1}, \mathrm{~T}_{2}$ are theories of $\mathcal{L}_{\mathrm{PA}}^{2}$ as introduced in Subsection 7.1 such that $\mathrm{T}_{2}$ comprises $\mathrm{T}_{1}$, then obviously $A \in \Pi_{n}\left(\mathrm{~T}_{1}\right)$ implies $A \in \Pi_{n}\left(\mathrm{~T}_{2}\right)$.

Proposition 7.14. Let $k, n \in \mathbb{N}$ and $\mathrm{T} \in\left\{\mathrm{ACA}_{0}, \Sigma_{1}^{1}-\mathrm{AC}_{0}\right\}$. Then we have the following.

(a) If $A \in \Pi_{k}^{1}(\mathrm{~T})$ holds with $k<n$, then we have $A \in \Pi_{n}^{1}(\mathrm{~T})$ and $\neg A \in \Pi_{n}^{1}(\mathrm{~T})$.

(b) $\Pi_{n}^{1}(\mathrm{~T})$ is closed under conjunction, disjunction, and universal quantification for number variables, i.e., we have that $A, B \in \Pi_{n}^{1}(\mathrm{~T})$ implies $A \circ B \in \Pi_{n}^{1}(\mathrm{~T})$ for $\circ \in\{\wedge, \vee\}$ and that $A \in \Pi_{n}^{1}(\mathrm{~T})$ implies $\forall x A \in \Pi_{n}^{1}(\mathrm{~T})$.

(c) $\Pi_{n+1}^{1}(\mathbf{T})$ is closed under universal quantification for set variables, i.e., we have that $A \in \Pi_{n+1}^{1}(\mathrm{~T})$ implies $\forall X A \in \Pi_{n+1}^{1}(\mathrm{~T})$. 
Proof. Straightforward by using essentially (ACA), see [Ran15, Proposition 7.36].

Corollary 7.15. Let $k, n \in \mathbb{N}$ and $\mathrm{T} \in\left\{\mathrm{ACA}_{0}, \Sigma_{1}^{1}-\mathrm{AC}_{0}\right\}$. Given $A_{0}, \ldots, A_{k} \in \Pi_{n}^{1}(\mathrm{~T})$ and $B \in \Pi_{n+1}^{1}(\mathrm{~T})$, we have $\forall \vec{x}\left(A_{0} \rightarrow \ldots \rightarrow A_{k} \rightarrow B\right) \in \Pi_{n+1}^{1}(\mathrm{~T})$.

Proof. Immediate by Proposition 7.14 and induction on $k \in \mathbb{N}$, while noting that $A_{k} \in \Pi_{n}^{1}(\mathrm{~T})$ implies $\neg A_{k} \in \Pi_{n+1}^{1}(\mathrm{~T})$, and that $A_{k} \rightarrow B$ is equivalent to $\neg A_{k} \vee B$.

\subsection{Embedding FIT into $\Pi_{3}^{1}-\mathrm{RFN}_{0}$}

In order to interpret within $\Pi_{3}^{1}-\mathrm{RFN}_{0}$ the applicative part of FIT, i.e., I. in Definition 3.10, we shall first implement the so-called canonical model $\mathfrak{P} \mathfrak{R}$ for this applicative part. It is built upon ordinary recursion theory and by using indices of partial recursive functions for interpreting the function symbol $\cdot$ of $\mathcal{L}_{\mathrm{FIT}}$. A thorough introduction to this construction and a more detailed treatment of the following (in a slightly different setting) will be elaborated in [FJS]. Without going into detail, we let $\mathbf{T}$ be the ternary, primitive recursive relation $\mathbf{T}$ according to Kleene's Normal Form Theorem, and let $\mathbf{U}$ be the corresponding unary primitive recursive (result-extracting) function. Reading $\{e\}$ as the partial recursive function indexed by the number $e$, we write $\{e\}\left(n_{1}, \ldots, n_{k}\right) \simeq m$ to denote $\exists x\left(\mathbf{T}\left(e,\left\langle n_{1}, \ldots, n_{k}\right\rangle, x\right) \wedge \mathbf{U}(x)=m\right)$ for $e, k, m, n_{1}, \ldots, n_{k} \in \mathbb{N}$. Furthermore, let $\Pi_{1}^{1}(x, y)$ with $x \neq y$ denote a universal $\Pi_{1}^{1}$ formula for $\Pi_{1}^{1}$ formulas that have one free variable, i.e., we have $\Pi_{1}^{1}(x, y) \in \Pi_{1}^{1}$ and for each $\mathcal{L}_{\mathrm{PA}}^{2}$ formula $A \in \Pi_{1}^{1}$ with $\mathrm{FV}(A)=\{y\}$, we have that $\exists x \forall y\left(\Pi_{1}^{1}(x, y) \leftrightarrow A\right)$ holds over $\mathrm{ACA}_{0} \cdot{ }^{12}$

Definition 7.16. In the abovementioned setting, we let $\mathbf{T}$ and $\mathbf{U}$ also denote the corresponding relation and function symbols in $\mathcal{L}_{\mathrm{PA}}$, and then we set $(\{a\}(b) \simeq c):=\exists x(\mathbf{T}(a, b, x) \wedge$ $\mathbf{U}(x)=c)$. Next, we assume an assignment of the constants $\mathrm{k}, \mathrm{s}$ of $\mathcal{L}_{\mathrm{FIT}}$ to numerals $\mathrm{k}^{\star}, \mathrm{s}^{\star}$ that have corresponding properties over $\mathrm{ACA}_{0}$ as described by the axiom group I.1. in definition 3.10. For the remaining constants $\mathrm{p}, \mathrm{p}_{0}, \mathrm{p}_{1}, 0, \mathrm{~s}_{\mathrm{N}}, \mathrm{p}_{\mathrm{N}}, \mathrm{d}_{\mathrm{N}}$ of $\mathcal{L}_{\mathrm{FIT}}$, we set $\mathrm{p}^{\star}$ to be the numeral of the (primitive recursive) function $(m, n) \mapsto\langle m, n\rangle ; \mathbf{p}_{i}^{\star}$ to be the numeral of $m \mapsto(m)_{i}$ for $i=0,1 ; 0^{\star}$ to be $0 ; \mathrm{s}_{\mathrm{N}}^{\star}$ to be the numeral of $m \mapsto m+1 ; \mathrm{p}_{\mathrm{N}}^{\star}$ to be the numeral of $m \mapsto m-1$ (i.e., the modified subtraction function such that $m \dot{-}_{\mathbb{N}} n=0$ holds if $m<\mathbb{N} n$ ); and $\mathrm{d}_{\mathrm{N}}^{\star}$ to be the numeral of the case distinction function, mapping $(k, l, m)$ to $l$ if $k=0$, otherwise to $m$.

The translation $\mathrm{V}_{t}^{\star}(x)$ of a $\mathcal{L}_{\mathrm{FIT}}$ term $t$ into the language of $\mathcal{L}_{\mathrm{PA}}$ (expressing that the value of $t$ equals $x$ ) is defined for variables $x \notin \mathrm{FV}(t)$ as follows: $t=x$ if $t$ is a variable, $t^{\star}=x$ if $t$ is a constant, and $\exists y, z\left(\mathrm{~V}_{r}^{\star}(y) \wedge \mathrm{V}_{s}^{\star}(z) \wedge\{y\}(z) \simeq x\right)$ if $t$ is rs. For each $\mathcal{L}_{\mathrm{FIT}}$ formula $A$, we let the $\mathcal{L}_{\mathrm{PA}}^{2}$ formula $A^{\star}$ be defined recursively on the build-up of $A$ as follows for every $x \notin \mathrm{FV}(A)$ :

$$
\begin{array}{rlrl}
(s=t)^{\star} & :=\exists x\left(\mathrm{~V}_{s}^{\star}(x) \wedge \mathrm{V}_{t}^{\star}(x)\right) & (t \in \mathrm{N})^{\star} & :=\exists x\left(\mathrm{~V}_{t}^{\star}(x)\right) \\
(t \in \mathrm{U})^{\star} & :=\exists x\left(\mathrm{~V}_{t}^{\star}(x) \wedge x \in \mathrm{U}\right) & (t \in \overline{\mathrm{N}})^{\star} & :=\mathrm{V}_{t}^{\star}(0) \wedge \neg \mathrm{V}_{t}^{\star}(0) \\
(t \in X)^{\star} & :=\exists x\left(\mathrm{~V}_{t}^{\star}(x) \wedge x \in X\right) & \left(\left\{x: B_{+}\right\}\right)^{\star}:=\exists x\left(\mathrm{~V}_{t}^{\star}(x) \wedge B^{\star}(z / x)\right) \\
(\neg B)^{\star} & :=\neg\left(B^{\star}\right) & (B \circ C)^{\star} & :=B^{\star} \circ C^{\star} \\
(Q z B)^{\star} & :=Q z B^{\star} & (Q X B)^{\star} & :=Q z\left(B^{\star}\left(\left(\Lambda a \cdot \Pi_{1}^{1}(z, a)\right) / X\right)\right)
\end{array}
$$

for $B_{+} \in$ For $^{+}, \circ \in\{\wedge, \vee, \rightarrow\}$ and $Q \in\{\forall, \exists\}$. For the case that $A$ is $t \in \mathbb{I}_{\mathbb{P}, \mathbb{Q}}$, we introduce first the following positive operator form (for any $\mathbb{P}, \mathbb{Q} \in \mathrm{Ty} \uparrow$ )

$$
\operatorname{Acc}_{\mathbb{P}, \mathbb{Q}}^{\star}:=\Lambda X \Lambda x \cdot(x \in \mathbb{P})^{\star} \wedge \forall y\left((y \in \mathbb{P})^{\star} \rightarrow(\langle y, x\rangle \in \mathbb{Q})^{\star} \rightarrow y \in X\right)
$$

\footnotetext{
${ }^{12}$ Bear in mind that this universal $\Pi_{1}^{1}$ formula shall include the unary relation symbol $U$ of $\mathcal{L}_{\mathrm{PA}}$ as a parameter.
} 
and note that $\mathbb{P}, \mathbb{Q}$ do not contain expressions of the form $\mathbb{I}_{\mathbb{P}^{\prime}, \mathbb{Q}^{\prime}}$. Eventually, we set

$$
\left(t \in \mathbb{I}_{\mathbb{P}, \mathbb{Q}}\right)^{\star}:=\forall X\left(\forall x\left(\operatorname{Acc}_{\mathbb{P}, \mathbb{Q}}^{\star}(X, x) \rightarrow x \in X\right) \rightarrow(t \in X)^{\star}\right)
$$

Furthermore, we tacitly assume in the definition of the translation $A^{\star}$ as usual a renaming of bound variables in order to avoid a clash of variables. Note also that the translation is meant to interpret type variables as $\Pi_{1}^{1}$ definable sets and that $I_{\mathbb{P}, \mathbb{Q}} \in$ Ty implies that $\mathbb{P}, \mathbb{Q}$ do not contain type variables (since $\mathbb{P}, \mathbb{Q} \in \mathrm{Ty} \uparrow$ ).

Lemma 7.17. Let $A \in \mathcal{L}_{\mathrm{FIT}}$, then $A^{\star}$ and $A$ have the same free variables.

Proof. This is clear from the definition of $A^{\star}$. We note that it is due to this lemma that we defined $(t \in \overline{\mathrm{N}})^{\star}$ as $\mathrm{V}_{t}^{\star}(0) \wedge \neg \mathrm{V}_{t}^{\star}(0)$ instead as $\neg(0=0)$.

Remark 7.18. For any $\mathbb{F} \in \mathrm{FT}$, consider the $\mathcal{L}_{\mathrm{PA}}^{2}$ class term $\mathcal{A}:=\Lambda z .(t z \in \mathbb{F})^{\star}$. In order to make later arguments more readable, we shall make the translation of the $\mathcal{L}_{\mathrm{FIT}}$ formula $\mathrm{Cl}_{\mathbb{P}, \mathbb{Q}}(\Lambda z . t z \in \mathbb{F})$ more explicit (cf., Notation 3.8):

$$
\left(\mathrm{Cl}_{\mathbb{P}, \mathbb{Q}}(\Lambda z . t z \in \mathbb{F})\right)^{\star} \text { is } \forall x\left(\operatorname{Acc}_{\mathbb{P}, \mathbb{Q}}^{\star}(\mathcal{A}, x) \rightarrow \mathcal{A}(x)\right)
$$

We defined $\mathrm{Cl}_{\mathbb{P}, \mathbb{Q}}(\Lambda z . t z \in \mathbb{F})$ in Section 3 in order to have the above representation that allows to use $\operatorname{Acc}_{\mathbb{P}, \mathbb{Q}}^{\star}$ in a intuitive way.

Lemma 7.19. Let $n \geq 0$. For each $\mathcal{L}_{\mathrm{FIT}}$ term $t$ and each $\mathcal{L}_{\mathrm{PA}}$ term $r$, the following holds.

(a) $\mathrm{V}_{t}^{\star}(x) \in \Pi_{0}^{1}$.

(b) $(t \in X)^{\star} \in \Pi_{0}^{1}$ and $\mathbb{P} \in \mathrm{Ty} \uparrow$ imply $(t \in \mathbb{P})^{\star} \in \Pi_{0}^{1}$.

(c) $B \in \Pi_{n}^{1}(\mathrm{~T})$ implies $\operatorname{Acc}_{\mathbb{P}, \mathbb{Q}}^{\star}(\mathcal{B}, r) \in \Pi_{n}^{1}(\mathrm{~T})$ for $\mathrm{T} \in\left\{\mathrm{ACA}_{0}, \Sigma_{1}^{1}-\mathrm{AC}_{0}\right\}, \mathcal{B}:=\Lambda a \cdot B(a)$ with $B \in \mathcal{L}_{\mathrm{PA}}^{2}$, and $\mathbb{P}, \mathbb{Q} \in \mathrm{Ty} \uparrow$. In particular, we have $\operatorname{Acc}_{\mathbb{P}, \mathbb{Q}}^{\star}(\mathcal{B}, r) \in \Pi_{0}^{1}$ in case of $B \in \Pi_{0}^{1}$.

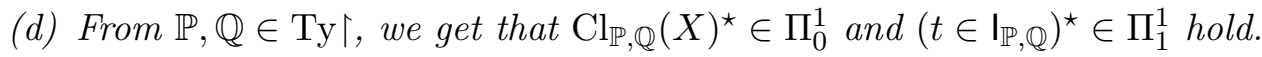

(e) $A \in$ For $^{+}$implies $A \in \Pi_{1}^{1}\left(\Sigma_{1}^{1}-\mathrm{AC}_{0}\right)$.

(f) $\mathbb{F} \in \mathrm{FT}$ implies $(t \in \mathbb{F})^{\star} \in \Pi_{2}^{1}\left(\Sigma_{1}^{1}-\mathrm{AC}_{0}\right)$.

Proof. For (a): This follows easily after inspecting the definition of $\mathrm{V}_{t}^{\star}(x)$. For (b): $(t \in$ $X)^{\star} \in \Pi_{0}^{1}$ follows from (a). Given $\mathbb{P} \in \mathrm{Ty} \uparrow$, we first note that then by definition, it can only be the case that $\mathbb{P}$ is $\mathrm{N}, \overline{\mathrm{N}}$, or $\{x: A\}$ for some $A \in \mathrm{For}^{+}$such that $A$ does not contain any $\mathbb{I}_{\mathbb{P}^{\prime}, \mathbb{Q}^{\prime}}$ expression or type variable. By (a) and Definition 7.16 , one can easily verify that $(t \in \mathbb{P})^{\star} \in \Pi_{0}^{1}$ holds.

For (c): $\operatorname{Acc}_{\mathbb{P}, \mathbb{Q}}^{\star}(\mathcal{B}, r)$ translates to the formula $(r \in \mathbb{P})^{\star} \wedge \forall y\left((y \in \mathbb{P})^{\star} \rightarrow(\langle y, x\rangle \in\right.$ $\left.(\mathbb{Q})^{\star}(r / x) \rightarrow y \in \mathcal{B}\right)$ and then the claim follows from Proposition 7.14 and (b), using the assumption $B \in \Pi_{n}^{1}(\mathrm{~T})$ and that $\mathbb{P}, \mathbb{Q} \in \mathrm{Ty} \uparrow$ holds.

For (d): We have $\operatorname{Acc}_{\mathbb{P}, \mathbb{Q}}^{\star}(X, x) \in \Pi_{0}^{1}$ by the second claim of (c), and further with (b) and after inspecting $\left(\star-\mathbb{I}_{\mathbb{P}, \mathbb{Q}}\right)$ on page 30 and $\left(\star-\mathrm{Cl}_{\mathbb{P}, \mathbb{Q}}\right)$ on page 30 , the claim becomes clear.

For (e): We prove here a more general statement

$$
\left.\begin{array}{rl}
A \in \text { For }^{+} & \Longrightarrow A^{\star} \in \Pi_{1}^{1}\left(\Sigma_{1}^{1}-\mathrm{AC}_{0}\right) \\
\neg A \in \text { For }^{+} & \Longrightarrow \neg A^{\star} \in \Pi_{1}^{1}\left(\Sigma_{1}^{1}-\mathrm{AC}_{0}\right)
\end{array}\right\}
$$

and by induction on the build-up of the $\mathcal{L}_{\mathrm{FIT}}$ formula $A$. Now, let $A \in \mathrm{For}^{+}$or $\neg A \in \mathrm{For}^{+}$be given. Note that $A$ cannot be of the form $\forall X A_{0}$ or $\exists X A_{0}$ because of the definition of For ${ }^{+}$. 
1. Base case: If $A$ is of the form $t \in \mathrm{U}, t \downarrow$, or $s=t$, we have $A^{\star} \in \Pi_{0}^{1}$ and are done.

2. Step case $t \in \mathbb{P}:$ If $A$ is $t \in \mathbb{P}$ with $\mathbb{P} \in$ Ty, then $A \in$ For $^{+}$must hold. Because of (b), we also only need to consider the case where $\mathbb{P} \notin \mathrm{Ty} \uparrow$ and $\mathbb{P}$ is not a type variable. Hence, $\mathbb{P}$ is either of the form $\mathbb{I}_{\mathbb{P}^{\prime}, \mathbb{Q}^{\prime}}$ with $\mathbb{P}^{\prime}, \mathbb{Q}^{\prime} \in \mathrm{Ty} \uparrow$ or $\mathbb{P}$ is of the form $\{z: B\}$ for some $B \in$ For $^{+}$.

In the first case, we get $A^{\star} \in \Pi_{1}^{1}\left(\Sigma_{1}^{1}-\mathrm{AC}_{0}\right)$ from (d). For the second case, recall that $(t \in\{z: B\})^{\star}$ equals $\exists x\left(\mathrm{~V}_{t}^{\star}(x) \wedge B^{\star}(x / z)\right)$ and note that by the induction hypothesis for $(*)$ with $B(x / z)$, we get $A_{0}(U, x) \in \Pi_{0}^{1}$ for some set variable $U$ such that $B^{\star}(x / z)$ is equivalent to $\forall X A_{0}(X, x)$ over $\Sigma_{1}^{1}-\mathrm{AC}_{0}$. Hence $(t \in\{z: B\})^{\star}$ is equivalent to $\exists x \forall X\left(\mathrm{~V}_{t}^{\star}(x) \wedge A_{0}(X, x)\right)$.

Now it remains only to show that $\exists x \forall X\left(\mathrm{~V}_{t}^{\star}(x) \wedge A_{0}(X, x)\right)$ is equivalent to $\forall X A_{0}^{\prime}(X)$ over $\Sigma_{1}^{1}-\mathrm{AC}_{0}$ for $A_{0}^{\prime}(W):=\exists x\left(\mathrm{~V}_{t}^{\star}(x) \wedge A_{0}\left((W)_{x}, x\right)\right)$, while noting $\forall X A_{0}^{\prime}(X) \in \Pi_{1}^{1}$. One direction of the equivalence holds already over $\mathrm{ACA}_{0}$ : In order to show $A_{0}\left((X)_{y}, y\right)$ for some $y$ for any given set $X$, take $x$ that is given in $\exists x \forall X\left(\mathrm{~V}_{t}^{\star}(x) \wedge A_{0}(X, x)\right)$. Then use (ACA) to get $Z$ such that $z \in Z \leftrightarrow z \in(X)_{x}$ holds, then we get $\mathrm{V}_{t}^{\star}(x) \wedge A_{0}(Z, x)$, i.e., $\mathrm{V}_{t}^{\star}(x) \wedge A_{0}\left((X)_{x}, x\right)$. For the converse direction, we can work with the contraposition and apply $\left(\Sigma_{1}^{1}-\mathrm{AC}\right)$.

3. Otherwise: The remaining cases follow from standard arguments involving $\Sigma_{1}^{1}-\mathrm{AC}_{0}$, the induction hypothesis, and Proposition 7.14. We refer to [Ran15, Lemma 7.41] for details.

For (f): We prove this for $\mathbb{F} \in \mathrm{FT}$ with $\mathbb{F}=\mathbb{P}_{0} \rightarrow \ldots \rightarrow \mathbb{P}_{n}$ by induction on $n \in \mathbb{N}$ : If $n=0$ holds, then we have $\mathbb{F} \in \mathrm{Ty}$ and $(t \in \mathbb{F}) \in \mathrm{For}^{+}$, so we can use (e). If $n>0$ holds, then let $\mathbb{F}^{\prime}:=\mathbb{P}_{1} \rightarrow \ldots \rightarrow \mathbb{P}_{n}$. Now, $(t \in \mathbb{F})^{\star}$ translates to $\forall x\left((x \in \mathbb{P})^{\star} \rightarrow\left(t x \in \mathbb{F}^{\prime}\right)^{\star}\right)$. By (e) and the induction hypothesis, we get $(x \in \mathbb{P})^{\star} \in \Pi_{1}^{1}\left(\Sigma_{1}^{1}-\mathrm{AC}_{0}\right)$ and $\left(t x \in \mathbb{F}^{\prime}\right)^{\star} \in \Pi_{2}^{1}\left(\Sigma_{1}^{1}-\mathrm{AC}_{0}\right)$. By Corollary 7.15 , we get $(t \in \mathbb{F})^{\star} \in \Pi_{2}^{1}\left(\Sigma_{1}^{1}-\mathrm{AC}_{0}\right)$.

Theorem 7.20. $\Pi_{3}^{1}-\mathrm{RFN}_{0}$ proves $A^{\star}$ for every instance $A$ of (FT-ID).

Proof. Let $A$ be an instance of (FT-ID), say $\mathrm{Cl}_{\mathbb{P}, \mathbb{Q}}(\Lambda z . t z \in \mathbb{F}) \rightarrow t \in\left(\mathbb{I}_{\mathbb{P}, \mathbb{Q}} \rightarrow \mathbb{F}\right)$ with $\mathbb{F} \in \mathrm{FT}$. Similar as in $\left(\star-\mathrm{Cl}_{\mathbb{P}, \mathbb{Q}}\right)$ on page 30 , we have with $\mathcal{A}:=\Lambda z .(t z \in \mathbb{F})^{\star}$ that $A^{\star}$ translates to $\forall x\left(\operatorname{Acc}_{\mathbb{P}, \mathbb{Q}}^{\star}(\mathcal{A}, x) \rightarrow \mathcal{A}(x)\right) \rightarrow \forall x\left(\left(x \in \mathbb{I}_{\mathbb{P}, \mathbb{Q}}\right)^{\star} \rightarrow \mathcal{A}(x)\right)$ and therefore we assume (with a slight renaming of bound variables to make the following more readable) that $\forall y\left(\operatorname{Acc}_{\mathbb{P}, \mathbb{Q}}^{\star}(\mathcal{A}, y) \rightarrow\right.$ $\mathcal{A}(y))$ holds. Due to Lemma 7.19.(f), we know that a formula $B \in \Pi_{2}^{1}$ exists such that $\Sigma_{1}^{1}-\mathrm{AC}_{0} \vdash B \leftrightarrow \mathcal{A}(y)$ holds. For $\mathcal{B}:=\Lambda y$. $B$, we get from Corollary 7.15 and Lemma 7.19.(c) a formula $C \in \Pi_{3}^{1}$ such that

$$
\mathrm{ACA}_{0} \vdash C \leftrightarrow \forall y\left(\operatorname{Acc}_{\mathbb{P}, \mathbb{Q}}^{\star}(\mathcal{B}, y) \rightarrow \mathcal{B}(y)\right)
$$

holds. Note that this holds over $\mathrm{ACA}_{0}$ since we work with $B \in \Pi_{2}^{1}$ instead of $(t y \in \mathbb{F})^{\star}$. Moreover, we have over $\Sigma_{1}^{1}-\mathrm{AC}_{0}$ that our assumption $\forall y\left(\operatorname{Acc}_{\mathbb{P}, \mathbb{Q}}^{\star}(\mathcal{A}, y) \rightarrow \mathcal{A}(y)\right)$ is equivalent to $\forall y\left(\operatorname{Acc}_{\mathbb{P}, \mathbb{Q}}^{\star}(\mathcal{B}, y) \rightarrow \mathcal{B}(y)\right)$ and we proceed now by assuming that the conclusion in $A^{\star}$ is false and will derive a contradiction from this. So, let $a_{0}$ be such that

$$
\left(x \in \mathbb{I}_{\mathbb{P}, \mathbb{Q})^{\star}}\left(a_{0} / x\right) \wedge \neg \mathcal{A}\left(a_{0}\right)\right.
$$

holds and note that the formula $\neg \mathcal{A}\left(a_{0}\right)$ (which is $\neg(t z \in \mathbb{F})^{\star}\left(a_{0} / z\right)$ ) is equivalent over $\Sigma_{1}^{1}-\mathrm{AC}_{0}$ to $\neg B\left(a_{0} / y\right)$. Note that $\neg B\left(a_{0} / y\right)$ is equivalent to a $\Pi_{3}^{1}$ formula, and since we have $C \in \Pi_{3}^{1}$, there exists by Proposition 7.14 some $D \in \Pi_{3}^{1}$ that is provably equivalent over $\mathrm{ACA}_{0}$ to $C \wedge \neg B\left(a_{0} / y\right)$. Then due to Corollary 7.9 , we can work with $\Pi_{3}^{1}-\mathrm{RFN}_{0}$ to apply $\left(\Pi_{3}^{1}-\mathrm{RFN}\right)$ to $D$ and thus obtain an $\omega$-model $M$ of $\operatorname{ACA}_{0}$ such that $\neg B^{M}\left(a_{0} / y\right)$ and $\forall y\left(\operatorname{Acc}_{\mathbb{P}, \mathbb{Q}}^{\star}(\mathcal{B}, y)^{M} \rightarrow\right.$ $\left.\mathcal{B}(y)^{M}\right)$ hold. For the latter, the relativization to $M$ holds essentially because of the equivalence 
in (12) being provable over $\mathrm{ACA}_{0}$. Further, this formula unfolds by Definition 7.1 and the build-up of $\operatorname{Acc}_{\mathbb{P}, \mathbb{Q}}^{\star}(\mathcal{B}, y)$ to

$$
\forall y\left(\operatorname{Acc}_{\mathbb{P}, \mathbb{Q}}^{\star}\left(\Lambda y \cdot B^{M}, y\right) \rightarrow B^{M}\right)
$$

Since $B^{M}$ is arithmetical, (ACA) provides a set $X_{0}$ such that we have

$$
\forall y\left(y \in X_{0} \leftrightarrow B^{M}\right) \wedge \forall y\left(\operatorname{Acc}_{\mathbb{P}, \mathbb{Q}}^{\star}\left(X_{0}, y\right) \rightarrow y \in X_{0}\right)
$$

Now, after recalling $\left(\star-I_{\mathbb{P}, \mathbb{Q}}\right)$ on page 30 , we can instantiate $\left(x \in \mathbb{I}_{\mathbb{P}, \mathbb{Q}}\right)^{\star}\left(a_{0} / x\right)$ from $(13)$ with $X_{0}$ and then use (15) to obtain $\left(x \in X_{0}\right)^{\star}\left(a_{0} / x\right)$, i.e., $\exists z\left(\mathrm{~V}_{x}^{\star}(z) \wedge z \in X_{0}\right)\left(a_{0} / x\right)$. This formula is equivalent to $a_{0} \in X_{0}$ since $\mathrm{V}_{x}^{\star}(z)$ is just $x=z$. But then we get $B^{M}\left(a_{0} / y\right)$ by (15) which is a contradiction to $\neg B^{M}\left(a_{0} / y\right)$ that we previously obtained. So, we have proven the lemma.

Remark 7.21. In the previous proof, we consider (14) as the pivotal property for the used proof method because it allowed to internalize an argument within the $\omega$-model $M$. In particular, we needed that the positive operator form $\mathrm{Acc}_{\mathbb{P}, \mathbb{Q}}^{\star}$ has the property described by Lemma 7.19.(c) with $\mathrm{T}$ being $\mathrm{ACA}_{0}$. A conceptually similar proof in the setting of $\Pi_{2}^{1}-\mathrm{RFN}_{0}$ and using similar standard results from the area of subsystems of second order arithmetic can be found in [AR10], treating the embedding of the theory $\mathrm{ID}_{1}^{*}$ of positive induction into $\Pi_{2}^{1}-\mathrm{RFN}_{0}$.

Now, turning to the question if our proof method would also work for arbitrary positive operator forms $\mathfrak{A}$, we point out that a direct embedding of TID into $\Pi_{3}^{1}-\mathrm{RFN}_{0}$ can be carried out almost literally as the embedding of FIT into $\Pi_{3}^{1}-\mathrm{RFN}_{0}$. More precisely, the previous lemmas can be reformulated in a very similar way so that they work for TID as well. The pivotal property to make the proof work would again correspond to (14) and essentially because Acc in the setting of TID has a similar bounded complexity as $\operatorname{Acc}_{\mathbb{P}, \mathbb{Q}}^{\star}$ here. The latter means that for (12) in the proof of Theorem 7.20, we used that we had the property $\operatorname{Acc}_{\mathbb{P}, \mathbb{Q}}^{\star}(\Lambda y . B, x) \in$ $\Pi_{2}^{1}\left(\mathrm{ACA}_{0}\right)$ at hand for $B \in \Pi_{2}^{1}$, namely as provided by Lemma 7.19.(c).

Continuing from the perspective of TID, we shall consider for a moment its natural generalization $\operatorname{TID}^{\mathbf{f}}$ (where $\mathbf{f}$ stands for full) that allows for arbitrary arithmetical operator forms $\mathfrak{A}$. So, having $\mathfrak{A}$ instead of $\operatorname{Acc}_{\mathbb{P}, \mathbb{Q}}^{\star}$ or Acc in (12), it would not always be possible to obtain a property such as $\mathfrak{A}(\mathcal{B}, x) \in \Pi_{2}^{1}\left(\mathrm{ACA}_{0}\right)$, nor can we expect that $G^{\prime} \in \Pi_{2}^{1}$ exists that is equivalent over $\Sigma_{1}^{1}-\mathrm{AC}_{0}$ or $\Pi_{3}^{1}-\mathrm{RFN}_{0}$ to $\mathfrak{A}(\mathcal{B}, x)$. Comparing this with the mentioned embedding of ID* into $\Pi_{2}^{1}-\mathrm{RFN}_{0}$ from [AR10], we note that essentially only $\Pi_{1}^{1}$ formulas $B$ needed to be considered there, and since a formula such as $\mathfrak{A}(\Lambda z . B, t)$ can be proven to be equivalent over $\Sigma_{1}^{1}-\mathrm{AC}_{0}$ to a $\Pi_{1}^{1}$ formula $G^{\prime}$, one can continue the proof with this $G^{\prime}$.

For an embedding of $\operatorname{TID}^{\mathbf{f}}$ into $\Pi_{3}^{1}-\mathrm{RFN}_{0}$ where we cannot control anymore the syntactical complexity of the positive operator forms $\mathfrak{A}$, we apparently cannot directly apply the method of this section. As described in the conclusion of this article (see Section 8), we remark here that the desired upper bound for $\operatorname{TID}^{\mathbf{f}}$ can be obtained by turning to the setting of set-theory.

Theorem 7.22. Over $\Pi_{3}^{1}-\mathrm{RFN}_{0}$, the following holds.

(a) $A^{\star}$ holds for every formula $A$ from axiom group $\boldsymbol{I}$. of FIT.

(b) $A^{\star}$ holds for every instance $A$ of the $\mathrm{N}$-induction scheme (FT-Ind) of FIT.

(c) $A^{\star}$ holds for every instance $A$ of the comprehension scheme $\left(\mathrm{CA}^{+}\right)$of $\mathrm{FIT}$.

(d) $A^{\star}$ holds for every instance $A$ of the closure axiom (FT-Cl) of FIT. 
Proof. For (a): Note that according to Definition 7.16, the type $\mathrm{N}$ has no special role in the translation $A^{\star}$ of any of the formulas $A$ given in the axiom group I. of FIT. As mentioned in Definition 7.16, we assume a standard interpretation of the constants $\mathrm{k}$ and $\mathrm{s}$ with the properties that we need for such a translation to be adequate. It is well-known that the combinators are available as partial recursive functions in the sense given here. Moreover, it is also more or less obvious that the interpretation of the remaining constants has the properties needed to make the translation of the remaining formulas in axiom group I. go through.

For (b): Over $A C A_{0}$, we have that ( $\Pi_{3}^{1}$-RFN) implies transfinite induction for $\Pi_{2}^{1}$ formulas, and thus complete induction along the natural numbers for $\Pi_{2}^{1}$ formulas. For this, see in [Sim09] Theorem VIII.5.12 and in particular Exercise VIII.5.15, while noting there that $\Sigma_{4}^{1}-\mathrm{RFN}_{0}$ is equivalent to $\Pi_{3}^{1}-\mathrm{RFN}_{0}$. Now, let $A$ be an instance $t 0 \in \mathbb{F} \wedge(\forall x \in \mathrm{N})(t x \in \mathbb{F} \rightarrow$ $\left.t x^{\prime} \in \mathbb{F}\right) \rightarrow t \in(\mathrm{N} \rightarrow \mathbb{F})$ of the $\mathrm{N}$-induction scheme (FT-Ind) of FIT, where $\mathbb{F} \in$ FT holds. By setting $\mathcal{B}:=\Lambda z .(t z \in \mathbb{F})^{\star}$, we have that $A^{\star}$ is equivalent over $\operatorname{ACA}_{0}$ to $\mathcal{B}(0) \wedge \forall x(\mathcal{B}(x) \rightarrow$ $\mathcal{B}(x+1)) \rightarrow \forall x\left(\exists y\left(\mathrm{~V}_{x}^{\star}(y)\right) \rightarrow \mathcal{B}(x)\right)$ since $\mathcal{B}(x+1)$ is equivalent to $\left(t\left(\mathrm{~s}_{\mathrm{N}} x\right) \in \mathbb{F}\right)^{\star}$. For the latter, note that $\left(t\left(\mathrm{~s}_{\mathrm{N}} x\right) \in \mathbb{F}\right)^{\star}$ is $\exists y\left(\mathrm{~V}_{t\left(\mathrm{~s}_{\mathrm{N}} x\right)}^{\star}(y) \wedge(y \in \mathbb{F})^{\star}\right)$ and that this is equivalent to $\exists y, z_{1}, z_{2}\left(\mathrm{~V}_{t}^{\star}\left(z_{1}\right) \wedge\left\{\mathrm{s}_{\mathrm{N}}^{\star}\right\}(x) \simeq z_{2} \wedge\left\{z_{1}\right\}\left(z_{2}\right) \simeq y \wedge(y \in \mathbb{F})^{\star}\right)$ which again simplifies to $\exists y, z_{1}\left(\mathrm{~V}_{t}^{\star}\left(z_{1}\right) \wedge\left\{z_{1}\right\}(x+1) \simeq y \wedge(y \in \mathbb{F})^{\star}\right)$ and this is equivalent to $\mathcal{B}(x+1)$. Now arguing over $\Pi_{3}^{1}-\mathrm{RFN}_{0}$, we have that $\mathcal{B}(0) \wedge \forall x(\mathcal{B}(x) \rightarrow \mathcal{B}(x+1)) \rightarrow \forall x\left(\exists y\left(\mathrm{~V}_{x}^{\star}(y)\right) \rightarrow \mathcal{B}(x)\right)$ is equivalent to an instance of complete induction along the natural numbers for a $\Pi_{2}^{1}$ formula (use Lemma 7.19.(f)) and hence we are done.

For (c): If $A$ is an instance $y \in\{x: B\} \leftrightarrow B(y / x)$ of $\left(\mathrm{CA}^{+}\right)$with $B \in \mathrm{For}^{+}$, then $A^{\star}$ yields $\exists x\left(\mathrm{~V}_{y}^{\star}(x) \wedge B^{\star}\right) \leftrightarrow(B(y / x))^{\star}$, being equivalent to the tautology $(B(y / x))^{\star} \leftrightarrow(B(y / x))^{\star}$.

For $(\mathrm{d})$ : Let $A:=\mathrm{Cl}_{\mathbb{P}, \mathbb{Q}}\left(\Lambda z . z \in \mathbb{I}_{\mathbb{P}, \mathbb{Q}}\right)$ be an instance of $(\mathrm{FT}-\mathrm{Cl})$. Following $\left(\star-\mathrm{Cl}_{\mathbb{P}, \mathbb{Q})}\right.$ on page 30 and in order to show $A^{\star}$, assume for $\mathcal{A}:=\Lambda z \cdot\left(z \in \mathbb{I}_{\mathbb{P}, \mathbb{Q}}\right)^{\star}$ that we have $\operatorname{Acc}_{\mathbb{P}, \mathbb{Q}}^{\star}\left(\mathcal{A}, z_{0}\right)$ for some $z_{0}$, and we aim to prove $\mathcal{A}\left(z_{0}\right)$, i.e., $\left(\forall X\left(\forall x\left(\operatorname{Acc}_{\mathbb{P}, \mathbb{Q}}^{\star}(X, x) \rightarrow x \in X\right) \rightarrow(z \in\right.\right.$ $\left.\left.X)^{\star}\right)\right)\left(z_{0} / z\right)$ and in order to prove this, we shall prove $z_{0} \in X_{0}$, assuming $X_{0}$ such that $\forall x\left(\operatorname{Acc}_{\mathbb{P}, \mathbb{Q}}^{\star}\left(X_{0}, x\right) \rightarrow x \in X_{0}\right)$ holds. From the latter and the definition of $\left(z \in \mathbb{I}_{\mathbb{P}, \mathbb{Q}}\right)^{\star}$, we

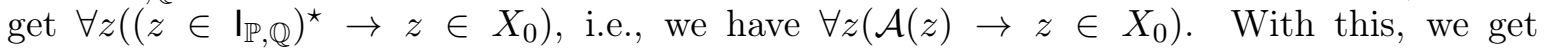
$\operatorname{Acc}_{\mathbb{P}, \mathbb{Q}}^{\star}\left(\mathcal{A}, z_{0}\right) \rightarrow \operatorname{Acc}_{\mathbb{P}, \mathbb{Q}}^{\star}\left(X_{0}, z_{0}\right)$ through the positivity of the operator form $\operatorname{Acc}_{\mathbb{P}, \mathbb{Q}}^{\star}$. Now, $\operatorname{Acc}_{\mathbb{P}, \mathbb{Q}}^{\star}\left(X_{0}, z_{0}\right)$ follows from assumption $\operatorname{Acc}_{\mathbb{P}, \mathbb{Q}}^{\star}\left(\mathcal{A}, z_{0}\right)$ and then the remaining goal $z_{0} \in X_{0}$ follows from assumption $\forall x\left(\operatorname{Acc}_{\mathbb{P}, \mathbb{Q}}^{\star}\left(X_{0}, x\right) \rightarrow x \in X_{0}\right)$.

Corollary 7.23. FIT $\vdash A$ implies $\Pi_{3}^{1}-\mathrm{RFN}_{0} \vdash A^{\star}$ for all $A \in \mathcal{L}_{\mathrm{FIT}}$.

Proof. By Theorems 7.20 and 7.22, one only has to show that the logical part of FIT embeds into $\Pi_{3}^{1}-\mathrm{RFN}_{0}$, i.e., that LPT $\vdash A$ implies $\Pi_{3}^{1}-\mathrm{RFN}_{0} \vdash A^{\star}$. The proof is by induction on the definition of the derivability notion LPT $\vdash A$ that may have been chosen in Definition 3.9 with respect to any sound Hilbert calculus. See [Ran15, Corollary 7.45] for details.

\section{Conclusion and Further Remarks}

Recapitulating the results of this article, we obtained theories FIT and TID that both have the small Veblen ordinal $\vartheta \Omega^{\omega}$ as their proof-theoretic ordinal, while FIT is a natural extension of Feferman's two-sorted theory $\mathrm{QL}\left(\mathrm{F}_{0}-\mathrm{I} \mathrm{R}_{N}\right)$ from [Fef92] and TID becomes from this perspective a natural subsystem of $I D_{1}$. Moreover, we used techniques from the realm of predicative prooftheory in order to obtain a wellordering proof for TID (and hence for FIT). 
With regard to the upper-bound results, we embedded FIT into the subsystem $\Pi_{3}^{1}-\mathrm{RFN}_{0}$ of second order arithmetic, while exploiting the $\Pi_{1}^{1}$ definability of a least fixed point in such a setting. This method can be used almost literally for embedding TID directly into $\Pi_{3}^{1}-\mathrm{RFN}_{0}$ (see [Ran15, Chapter 9] for this). This approach seems to fail though if we extend TID to a theory TID $^{\mathbf{f}}$ for general typed inductive definitions with the full range of positive arithmetical operator forms (as described in Remark 7.21). A way to avoid this obstacle is to shift the setting to set-theory rather than subsystems of second order arithmetic, namely by exploiting the $\Sigma_{1}$ definability of a least fixed point. Working then in $\mathrm{KP} \omega^{-}+\Pi_{2}$-Found from [Rat92] (i.e., Kripke-Platek set-theory with a restricted axiom scheme for foundation) shall suffice to get an analog result as for FIT which we can apply to the theory TID $^{\mathbf{f}}$. Summing up, what we gain from these embeddings is that extending TID to the theory $\operatorname{TID}^{\mathbf{f}}$ retains the proof-theoretic upper bound $\vartheta \Omega^{\omega}$ because $\mathrm{KP} \omega^{-}+\Pi_{2}$-Found has by [Rat92] the same proof-theoretic strength as $\Pi_{2}^{1}-B_{0}$. Since TID trivially embeds into TID $^{\mathbf{f}}$ and as depicted in Figure 2, we get that TID $^{\mathbf{f}}$ has the same proof-theoretic strength as TID (in a similar way as $\mathrm{ID}_{1}$ and its restriction $\mathrm{ID}_{1}$ (Acc) to accessible part arithmetical operator forms correspond, see also [BFPS81]).

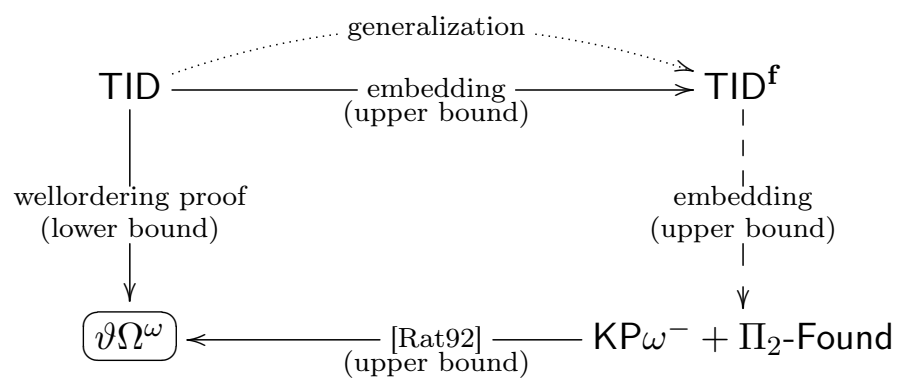

Figure 2: Generalization of TID to the full theory TID $^{\mathbf{f}}$

We finish with some remarks and conjectures on how to extend the proof-methods from Sections 6 and 7 in order to analyze stronger systems: First, the collections of formulas $\operatorname{Pos}_{0}$ and $\operatorname{Pos}_{1}$ suggest already a generalization to collections $\operatorname{Pos}_{n}$ for any $n \geq 2$ in the sense that the correspondence of $\operatorname{Pos}_{1}$ to function types of the form $\mathbb{P}_{1} \rightarrow \ldots \rightarrow \mathbb{P}_{k}$ for each $k \in \mathbb{N}$ (i.e., "level-1-functional types") passes over to $\mathrm{Pos}_{2}$ being in correspondence to level-2-functional types $\mathbb{F}_{1} \rightarrow \ldots \rightarrow \mathbb{F}_{k}$ for each $k \in \mathbb{N}$, and similar for $n>2$. Such theories are essentially considered in [Ran15] and named $\operatorname{TID}_{n}$. Moreover, intermediate systems TID $_{n}^{+}$are considered that are located between $\operatorname{TID}_{n}$ and $\mathrm{TID}_{n+1}$, and which arise essentially by adding a rule of inference $\left(\mathrm{TID}^{+}\right)$of the form

$$
\left(\mathrm{TID}^{+}\right) \frac{P_{\triangleleft} t}{\operatorname{Prog}_{\triangleleft}(\mathcal{B}) \rightarrow \mathcal{B}(t)}
$$

for arbitrary $\mathcal{B} \in \mathcal{L}_{\text {TID }}$ and for each binary relation symbol $\triangleleft \in \mathcal{L}_{\mathrm{PA}}$ and each term $t$. As shown in [Ran15], TID ${ }_{1}^{+}$has the strength of the large Veblen ordinal $\vartheta \Omega^{\Omega}$ and $\left(\mathrm{TID}^{+}\right.$) corresponds to a variant of a bar rule. This and the results of [Pro17] suggest that by adding the usual bar rule (BR) to the theory $\Pi_{3}^{1}-\mathrm{RFN}_{0}$ with strength of the small Veblen ordinal $\vartheta \Omega^{\omega}$, we obtain a theory $\Pi_{3}^{1}-R^{2} N_{0}+(B R)$ with strength of the large Veblen ordinal $\vartheta \Omega^{\Omega}$.

Concerning the wellordering proof for $\mathrm{TID}_{1}^{+}$in [Ran15, Chapter 11], we remark here that it is based on the methods from Section 6 by using Klammersymbols instead of finitary Veblen 
functions and by internalizing the arity of the finitary Veblen functions within the theory. More precisely and as an informal and intuitive explanation why Corollary 6.15 is the best we can expect from TID, we mention that the method used in the proof of Theorem 6.12 relied on an external representation of the finite list of arguments that the finitary Veblen function is applied to. In particular, induction in the meta-theory has been applied to cope with arbitrary but finite lists of arguments. The proof of Theorem 6.12 is designed for the theory TID, and in order to use it to get beyond the small Veblen ordinal, for instance by working with infinitary Veblen functions or Klammersymbols, the first step is to internalize the proof and deal with non-standard argument positions (for which we do not have a denotation in the meta-theory).

For more details and further remarks on the other theories, we refer to [Ran15]. The use of a higher-type functional for iterating the fixed-point construction on Klammersymbols allows to extend the ordinal notation system. Endowed with stronger induction principles that become available in the theories $\operatorname{TID}_{n}$ and $\mathrm{TID}_{n}^{+}$, this may lead towards higher ordinals via wellordering proofs based on the accessible part of the primitive recursive ordering of the new ordinal notation system.

\section{A. Appendix: The Ordinal Notation System $(\mathrm{OT}, \prec)$}

For carrying out the wellordering proofs in TID, we fix a primitive recursive notation system $(\mathrm{OT}, \prec)$ for ordinals below the small Veblen ordinal, based on codes of natural numbers. For a full definition and proofs, see [Ran15, Chapter 3]. (OT, $\prec)$ is based on Lemma 5.19 (i.e., essentially on (7.1)-(7.4) in [Sch54]) and its representation is inspired by [Buc05].

We point out that the definition and properties of $(\mathrm{OT}, \prec)$ can be formalized and established within PA and hence within TID. Due to constraints on the length of this article, we hide these things and establish here only the minimal technical information needed in order to be able to carry out the wellordering proofs in Section 6 in a formally satisfying way. In particular, we provide Section 5 as the set-theoretic intuition for those proofs and we refer to [Ran15] for the full definition of $(\mathrm{OT}, \prec)$, i.e., its formalization within $\mathcal{L}_{\mathrm{PA}}$, the (technically cumbersome) encoding and verification within PA of crucial primitive recursive properties (e.g., that (OT, $\prec$ ) is a strict total order), and the set-theoretic interpretation of $(\mathrm{OT}, \prec)$ by means of a function о: $\mathrm{OT} \rightarrow$ On as described in Section 5.2.

This appendix contains the formal definition and central properties of fundamental sequences for ordinal notations because the proofs of Section 6 are centrally based on these. In order to be able to parse this definition, we stress that OT consists of the following codes for ordinals:

- 0 for the zero ordinal;

- $\phi \bar{a}^{(n+1)}$ denoting a code for the intended application of the finitary Veblen function $\varphi$ to given $a_{1}, \ldots, a_{n+1} \in$ OT with $a_{1} \neq 0$ and where $a_{1}, \ldots, a_{n+1}$ suffice certain (normalform) conditions that are motivated by Lemma 5.19 ;

- $a \oplus b$ denoting a code for the intended sum of ordinals in Cantor normal form, where essentially $a$ is of the form $\phi \bar{a}^{(n+1)}$ and $b \neq 0$.

Suc and Lim denote primitive recursive sets of codes for successor ordinals and limit ordinals, respectively. As already mentioned in Section 5.2, ordinal arithmetic is simulated on such ordinal notations by making use of primitive recursive operations: We have $\tilde{+}: \mathrm{OT} \times \mathrm{OT} \rightarrow \mathrm{OT}$ and $\tilde{\varphi}_{n+1}: \mathrm{OT}^{n+1} \rightarrow$ OT that correspond to ${ } \mathrm{On}_{\mathrm{n}}: \mathrm{On} \times \mathrm{On} \rightarrow \mathrm{On}$ and $\varphi_{n+1}: \mathrm{On}^{n+1} \rightarrow \mathrm{On}$ 
from Section 5. We just write $\tilde{\varphi}$ instead of $\tilde{\varphi}_{n+1}$ if the arity is clear from the context. Further, let $\tilde{1}:=\phi 0$ and $\tilde{\omega}^{a}:=\tilde{\varphi}(a)$ for each $a \in \mathrm{OT}$. Also, we have $\tilde{:}:$ OT $\times \mathbb{N} \rightarrow$ OT to denote finite addition (encoded via $\oplus$ ) of the same ordinal notation, i.e., $a^{\sim} 0:=0$ and $a \tilde{r}(x+\mathbb{N} 1):=a \oplus(a \tilde{a} x)$ for all $a \in$ OT and $x \in \mathbb{N}$.

Definition A.1. Fundamental sequences for ordinal notations $d \in \mathrm{OT}$ are defined within PA by means of a binary primitive recursive function $L$ whose defining equations are described as follows, where $a, x$ range over natural numbers and where we write $a[x]$ to denote $L(a, x)$.

- If $d=0$ or $d \notin \mathrm{OT}$, then $d[x]:=0$

- If $d \in$ Suc with $d=d_{0} \tilde{+} \tilde{1}$, then $d[x]:=d_{0}$

- If $d \in \operatorname{Lim}$ and $d=a \oplus b$ with $a \in \mathrm{OT}$ and $b \in \operatorname{Lim}$, then $d[x]:=a \tilde{+} b[x]$

- If $d \in \operatorname{Lim}$ and $d=\phi a$ with $a \neq 0$, then $d[x]:= \begin{cases}\tilde{\omega}^{a_{0}} \tilde{r}(x+\mathbb{N} 1) & \text { if } a=a_{0} \tilde{+} \tilde{1} \\ \tilde{\omega}^{a[x]} & \text { otherwise }\end{cases}$

- If $d \in \operatorname{Lim}$ with $d=\phi \bar{a}^{(m)} b \overline{0}^{(k)} c$ for some $\bar{a}^{(m)}, b, c \in \mathrm{OT}$ with $b \neq 0$ and $m, k \in \mathbb{N}$, then

$$
\begin{aligned}
d[0]:= \begin{cases}\tilde{\varphi}\left(\bar{a}^{(m)}, b, \overline{0}^{(k)}, c[0]\right) & \text { if } c \in \operatorname{Lim} \\
\tilde{\varphi}\left(\bar{a}^{(m)}, b[0], \overline{0}^{(k+1)}\right) & \text { if } c=0 \text { and } b \in \operatorname{Lim} \\
\tilde{1} & \text { if } c=0 \text { and } b \in \operatorname{Suc} \\
\tilde{\varphi}\left(\bar{a}^{(m)}, b, \overline{0}^{(k)}, c[0]\right) \tilde{+} \tilde{1} & \text { otherwise (if } c \in \operatorname{Suc)}\end{cases} \\
d\left[x+_{\mathbb{N}} 1\right]:= \begin{cases}\tilde{\varphi}\left(\bar{a}^{(m)}, b, \overline{0}^{(k)}, c[x+\mathbb{N} 1]\right) & \text { if } c \in \operatorname{Lim} \\
\tilde{\varphi}\left(\bar{a}^{(m)}, b[x+\mathbb{N} 1], \overline{0}^{(k+1)}\right) & \text { if } c=0 \text { and } b \in \operatorname{Lim} \\
\tilde{\varphi}\left(\bar{a}^{(m)}, b[x], d[x], \overline{0}^{(k)}\right) & \text { otherwise (if } c \in \operatorname{Suc} \text { or }(c=0 \text { and } b \in \operatorname{Suc}))\end{cases}
\end{aligned}
$$

\section{References}

[AR10] Bahareh Afshari and Michael Rathjen. A note on the theory of positive induction, ID ${ }_{1}^{*}$. Archive for Mathematical Logic, 49:275-281, 2010. 10.1007/s00153-009-0168-9.

[Bee85] Michael J. Beeson. Foundations of Constructive Mathematics: Metamathematical studies. Springer Verlag, Berlin, Heidelberg, New York, 1985.

[BFPS81] Wilfried Buchholz, Solomon Feferman, Wolfram Pohlers, and Wilfried Sieg. Iterated Inductive Definitions and Subsystems of Analysis: Recent Proof-Theoretical Studies, volume 897 of Lecture Notes in Mathematics. Springer Verlag, Berlin, Heidelberg, New York, 1981.

[Bri75] Jane Bridge. A simplification of the Bachmann method for generating large countable ordinals. The Journal of Symbolic Logic, 40(2):171-185, 1975.

[BS88] Wilfried Buchholz and Kurt Schütte. Proof Theory of Impredicative Subsystems of Analysis, volume 2 of Studies in Proof Theory Monographs. Bibliopolis, Napoli, 1988. 
[Buc05] Wilfried Buchholz. Prädikative Beweistheorie (Predicative proof theory). Lecture notes, University of Munich, 2004-2005.

[Buc16] Wilfried Buchholz. A survey on ordinal notations around the Bachmann-Howard ordinal. In Reinhard Kahle, Thomas Strahm, and Thomas Studer, editors, Advances in Proof Theory, pages 1-29. Birkhaeuser, Springer Basel, 2016.

[Can86] Andrea Cantini. On the relation between choice and comprehension principles in second order arithmetic. The Journal of Symbolic Logic, 51(2):360-373, 1986.

[Fef79] Solomon Feferman. Constructive theories of functions and classes. In Dirk van Dalen Maurice Boffa and Kenneth Mcaloon, editors, Logic Colloquium '78 Proceedings of the colloquium held in Mons, volume 97 of Studies in Logic and the Foundations of Mathematics, pages 159-224. Elsevier, 1979.

[Fef92] Solomon Feferman. Logics for termination and correctness of functional programs, II. Logics of strength PRA. In Peter Aczel, Harold Simmons, and Stanley S. Wainer, editors, Proof Theory, pages 195-225. Cambridge University Press, 1992.

[FJS] Solomon Feferman, Gerhard Jäger, and Thomas Strahm. Foundations of Explicit Mathematics. Book in preparation.

[Jäg05] Gerhard Jäger. Metapredicative and explicit Mahlo: a proof-theoretic perspective. In Rene Cori, Alexander Razborov, Stevo Todorcevic, and Carol Wood, editors, Proceedings of Logic Colloquium '00, volume 19 of Association of Symbolic Logic Lecture Notes in Logic, pages 272-293. AK Peters, 2005.

[JKSS99] Gerhard Jäger, Reinhard Kahle, Anton Setzer, and Thomas Strahm. The prooftheoretic analysis of transfinitely iterated fixed point theories. The Journal of Symbolic Logic, 64(1):53-67, 1999.

[JS99] Gerhard Jäger and Thomas Strahm. Bar induction and $\omega$ model reflection. Annals of Pure and Applied Logic, 97(1-3):221-230, 1999.

[JS05] Gerhard Jäger and Thomas Strahm. Reflections on reflections in explicit mathematics. Annals of Pure and Applied Logic, 136(1-2):116-133, 2005. Festschrift on the occasion of Wolfram Pohlers' 60th birthday.

[Pro06] Dieter Probst. The proof-theoretic analysis of transfinitely iterated quasi least fixed points. The Journal of Symbolic Logic, 71(3):721-746, 2006.

[Pro17] Dieter Probst. Modular Ordinal Analysis of Subsystems of Second-Order Arithmetic of Strength up to the Bachmann-Howard Ordinal. Habilitation, Universität Bern, 2017. Submitted.

[Ran15] Florian Ranzi. From a Flexible Type System to Metapredicative Wellordering Proofs. PhD thesis, Universität Bern, 2015. http://www.iam.unibe.ch/ltgpub/2015/ ran15.pdf or http://dx.doi.org/10.7892/boris.75102.

[Rat92] Michael Rathjen. Fragments of Kripke-Platek set theory with infinity. In P. Aczel, H. Simmons, and S.S. Wainer, editors, Proof Theory. A selection of papers from the 
Leeds Proof Theory Programme 1990, pages 251-273. Cambridge University Press, 1992.

[RW93] Michael Rathjen and Andreas Weiermann. Proof-theoretic investigations on Kruskal's theorem. Annals of Pure and Applied Logic, 60:49-88, 1993.

[Sch54] Kurt Schütte. Kennzeichnung von Ordnungszahlen durch rekursiv erklärte Funktionen. Mathematische Annalen, 127:15-32, 1954. 10.1007/BF01361109.

[Sch92] Kurt Schütte. Beziehungen des Ordinalzahlensystems OT $(\vartheta)$ zur Veblen-Hierarchie. Unpublished notes, 1992.

[Sim09] Stephen G. Simpson. Subsystems of Second Order Arithmetic. Cambridge University Press, second edition, 2009. Cambridge Books Online.

[Str99] Thomas Strahm. First steps into metapredicativity in explicit mathematics. In S. Barry Cooper and John K. Truss, editors, Sets and Proofs, volume 258 of London Mathematical Society Lecture Notes, pages 383-402. Cambridge University Press, 1999.

[VRW17] Jeroen Van der Meeren, Michael Rathjen, and Andreas Weiermann. An ordertheoretic characterization of the Howard-Bachmann-hierarchy. Archive for Mathematical Logic, 56(1-2):79-118, February 2017. 